\title{
A SPECTROSCOPIC EXAMINATION OF ACCRETION DIAGNOSTICS FOR NEAR SOLAR MASS STARS IN IC 348
}

\author{
S. E. DAнM ${ }^{1,2}$ \\ ${ }^{1}$ Department of Astronomy, California Institute of Technology, MS 105-24, Pasadena, CA 91125, USA \\ Received 2007 October 31; accepted 2008 April 18; published 2008 June 24
}

\begin{abstract}
High-resolution optical and moderate-resolution near-infrared spectra were obtained for 40 near solar mass $(\sim 2.0$ $0.5 M_{\odot}$ ) members of the $2-3 \mathrm{Myr}$ old cluster IC 348 in order to examine established accretion diagnostics and the coupling between inner disk gas and hot, micron-sized dust grains inferred from thermal and mid-infrared excesses. The stellar sample was drawn from the cluster census of Luhman in 2003 with membership being confirmed by radial velocity analysis and the presence of strong Li I $\lambda 6708$ absorption. Of the stars included in this survey, 12 were classified by Lada in 2006 as hosting primordial, optically thick circumstellar disks, 5 as weak or transition disk systems, and 23 as non-excess stars using the measured slope of the stellar spectral energy distribution (SED) through the four Infrared Array Camera channels (3.6-8.0 $\mu \mathrm{m})$ of Spitzer Space Telescope. Using the velocity width of $\mathrm{H} \alpha$ as an accretion indicator, we find that 11 primordial disk candidates are suspected accretors, suggesting a strong correlation between gaseous inner disks and optically thick dust emission. Of the five weak or transition disk systems observed, two (L21 and L67) exhibit spectroscopic features indicative of accretion. The presence of gas within the inner disk of these systems, which are free of infrared excess emission shortward of $\sim 4.5 \mu \mathrm{m}$, may place constraints upon the physical mechanism responsible for inner disk clearing. Mass accretion rates $(\dot{M})$ were determined for all suspected accretors using continuum excess measurements near $\lambda 6500$ and established relationships between $\mathrm{He}$ I $\lambda 5876, \mathrm{H} \alpha, \mathrm{Ca}$ II $\lambda 8542, \mathrm{~Pa} \beta$, and $\mathrm{Br} \gamma$ line fluxes and accretion luminosity. $\dot{M}$ values were found to range from $\log \dot{M}=-8.7$ to $-7.2 M_{\odot} \mathrm{yr}^{-1}$, with a median value of $-8.1 M_{\odot} \mathrm{yr}^{-1}$. Magnetospheric accretion models of $\mathrm{H} \alpha, \mathrm{Pa} \beta$, and $\mathrm{Br} \gamma$ emission by Muzerolle et al. and Kurosawa et al. are found to be in relative agreement with observed fluxes and derived $\dot{M}$ estimates. He I $\lambda 10830$ with its metastable lower level is confirmed to be a critical indicator of magnetospheric accretion, although deep subcontinuum absorption profiles often associated with its emission are not fully understood. No statistically significant correlation is found between $\dot{M}$ and the slope of the SED from 3.6 to $8.0 \mu \mathrm{m}$ or from 8.0 to $24.0 \mu \mathrm{m}$; however, the small number of suspected accretors examined does not allow broader conclusions to be drawn.
\end{abstract}

Key words: accretion, accretion disks - open clusters and associations: individual (IC 348) - stars: formation stars: pre-main sequence

Online-only material: color figures

\section{INTRODUCTION}

Following the initial gravitational collapse of a molecular cloud core, most protostars are surrounded by optically thick disks of remnant molecular gas and dust, the progenitors of planetary systems. Growing empirical evidence from groundbased near and thermal infrared observations and from Spitzer Space Telescope mid-infrared photometry and spectroscopy suggests that primordial disks evolve rapidly, with just a few percent of stars retaining micron-sized dust within the inner AU beyond ages of $\sim 10 \mathrm{Myr}$ (Uchida et al. 2004; Silverstone et al. 2006). By inference, giant planet formation must occur over similar timescales, before molecular gas is significantly depleted from the disk. One striking discovery has been the strong dependence of primordial disk lifetimes upon stellar mass, such that stars of solar mass or less retain optically thick disks for longer periods than their more massive counterparts (Lada et al. 2006; Carpenter et al. 2006; Dahm \& Hillenbrand 2007). The immediate implication is that longer timescales may be available for the growth of planetesimals around these lowmass, pre-main-sequence stars. Critical for understanding disk

\footnotetext{
2 Visiting Astronomer at the Infrared Telescope Facility, which is operated by the University of Hawaii under Cooperative Agreement No. NCC 5-538 with the National Aeronautics and Space Administration, Science Mission Directorate, Planetary Astronomy Program.
}

structure and evolution is knowledge of the spatial distribution of gas as primordial disks transition to optically thin debris disks. Accretion is one definitive indicator of inner disk gas, and the mass accretion rate, $\dot{M}$, provides a critical constraint for disk evolution models and potential disk clearing mechanisms.

Accretion processes in pre-main-sequence stars are generally inferred from irregular variability, blue continuum excesses, broadened emission lines of $\mathrm{H} \mathrm{I}, \mathrm{He} \mathrm{I}$, and neutral or ionized metals, or from the presence of forbidden emission generally associated with outflows or disk winds (Gullbring et al. 1998; Hamann \& Persson 1992; Edwards et al. 1994; Cabrit et al. 1990). Analysis of high dispersion spectra provides a powerful means of establishing the presence of gaseous accretion and determining $\dot{M}$ for even weakly accreting systems. Muzerolle et al. (1998a) examine emission line profiles and fluxes of $\mathrm{H} \alpha$, He I $\lambda 5876$, O I $\lambda \lambda 7773,8446$ and the Ca II near-infrared triplet of several members of the Taurus-Auriga star-forming region having well-established $\dot{M}$ values from spectrophotometric, blue continuum excess measurements (Valenti et al. 1993; Gullbring et al. 1998). Their findings suggest that several of these prominent emission features are strongly correlated with $\dot{M}$ through several orders of magnitude, to below $\log \dot{M} \sim$ $-9 M_{\odot} \mathrm{yr}^{-1}$. Radiative transfer magnetospheric accretion models of H I emission lines by Muzerolle et al. (1998b; 2001) and Kurosawa et al. (2006) have also been developed to examine 


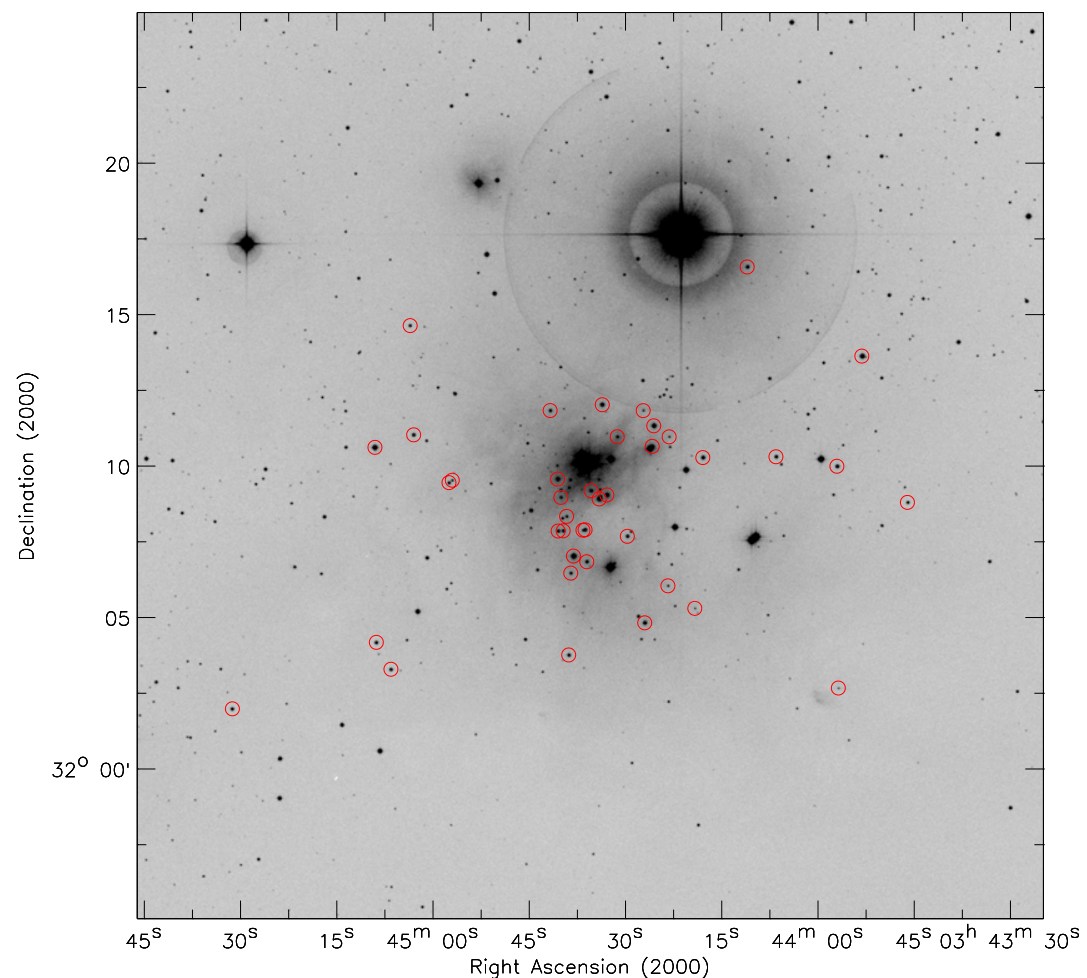

Figure 1. A $30^{\prime} \times 30^{\prime}$ Second Palomar Observatory Sky Survey (POSS II) red image of IC 348 obtained from the DSS. HD 281159 is the luminous B5 cluster member approximately centered in the image. $o$ Per, the 4th mag B1 III star lying to the northwest of the image center is a member of the Per OB2 association. The 40 stars of the membership sample are circled in red and represent a significant fraction $(\sim 87 \%)$ of the cluster population within this critical range of spectral types from G0 to $\mathrm{M} 0\left(\sim 2.0-0.5 M_{\odot}\right)$. At the age of IC $348(2-3 \mathrm{Myr})$, a solar mass star is expected to have a spectral type of $\sim \mathrm{K} 5-\mathrm{K} 7$.

(A color version of this figure is available in the online journal)

the effect of gas temperature, magnetospheric radii, and disk winds upon predicted line fluxes. These hydrogen emission models have been used with varying degrees of success to reproduce the broad range of $\mathrm{H} \alpha$ morphologies observed in classical T Tauri stars (CTTSs). In heavily extincted regions where blue continuum excess observations are not practicable, the need for infrared accretion diagnostics provided the impetus for Muzerolle et al.'s (1998c) investigation of $\mathrm{Pa} \beta$ and $\mathrm{Br} \gamma$ emission. They find both features to be strongly correlated with accretion luminosity, and consequently $\dot{M}$. In this investigation, several of these diagnostics are examined in order to probe accretion activity among the near solar analog population of the young (2-3 Myr) cluster IC 348.

IC 348 is a nearby $(\sim 320 \mathrm{pc})$ Galactic cluster lying at the northern edge of the Perseus molecular ridge (Herbig 1998). The cluster is exceptionally well-studied at all wavelengths from X-rays (Preibisch et al. 1996; Preibisch \& Zinnecker 2001, 2002) to the millimeter (Carpenter 2002; Eisloffel et al. 2003). Herbig (1954) first surveyed the cluster using the slitless grating spectrograph on the Crossley reflector at Lick Observatory, finding 16 low-luminosity $\mathrm{H} \alpha$ emission stars clustered around its brightest member, the B5 dwarf HD 281159. Follow-up observations with the wide-field grism spectrograph (WFGS) on the University of Hawaii $2.2 \mathrm{~m}$ telescope on Mauna Kea identified 110 emission line stars having $R$-band mag $<19$ (Herbig 1998). Lada \& Lada (1995) completed an extensive near-infrared $(J H K)$ imaging survey of IC 348, detecting 380 distinct sources, the majority of which were assumed to be cluster members. The ROSAT and Chandra X-ray observations of the cluster by Preibisch et al. (1996) and Preibisch \& Zinnecker $(2001,2002)$ have also added considerably to the cluster population. Combining these earlier surveys with their own deep $I$ - and $Z$-band photometry, Luhman et al. (2003) compiled a census for the cluster that is very likely complete for stars more massive than $\sim 0.3 M_{\odot}$. In total, some 288 cluster members, 23 of which are likely brown dwarfs, have been identified.

Using the Luhman et al. (2003) cluster census, Lada et al. (2006) examine deep Spitzer IRAC and Multi-band Imaging Photometer for Spitzer (MIPS) photometry for the nearly 300 candidate members of IC 348 . Their findings suggest that $30 \%$ of the stars examined possess primordial, optically thick circumstellar disks while another $\sim 20 \%$ exhibit weak or optically thin disk emission. Classification of disk structure was based upon the slope of the spectral energy distribution (SED) relative to that predicted for pure stellar photospheres through the four IRAC channels. Lada et al. (2006) conclude that disk frequency is strongly mass dependent, finding that $19 \%$ (11\% primordial, $8 \%$ weak) of stars earlier than K6, 56\% (47\% primordial, 9\% weak) of K6-M2 type stars, and 54\% (28\% primordial, 26\% weak) of M2-M6 type stars retain circumstellar disks. Their distinction between optically thick and optically thin disk emission, however, used identical slope values for all IC 348 members regardless of spectral type. This potential problem for late-type $(\geqslant M 3)$ stars has no impact upon the results of this survey given the relatively early spectral types examined here.

In this work we present the results of a high-resolution optical and moderate-resolution near-infrared spectroscopic survey of 40 members of IC 348 spanning a range in spectral type from G0 to M0 $\left(\sim 2.0-0.5 M_{\odot}\right)$. Shown in Figure 1 is a $30^{\prime} \times 30^{\prime}$ Second Palomar Observatory Sky Survey (POSS II) red image 
of IC 348 reproduced from the Digitized Sky Survey (DSS), which illustrates the distribution of the membership sample across the cluster region. HD 281159, the luminous B5 V binary, lies approximately centered in the image while $o$ Per, the 4th mag B1 III member of the Per OB2 association, lies to the northwest. The 40 stars of the observed sample represent a significant fraction of the cluster population within this critical range of spectral types that includes young solar analogs (at the age of IC 348 a solar mass star will possess a $\sim \mathrm{K} 5-\mathrm{K} 7$ spectral type). In the following sections we discuss the optical and infrared spectroscopic observations made, the observed sample and membership confirmation, various diagnostics of accretion used to establish the presence of infalling gas, and the determination of mass accretion rates using extinctioncorrected, emission line fluxes for $\mathrm{H} \alpha$, He I $\lambda 5876$, Ca II $\lambda 8542$, $\mathrm{Pa} \beta$, and $\mathrm{Br} \gamma$. We then examine the coupling between gaseous accretion and the presence of hot, micron-sized dust grains within the inner disk responsible for thermal and mid-infrared excesses inferred from the Spitzer IRAC and MIPS photometric survey of Lada et al. (2006). Finally we discuss accretion among weak or transition disk candidates included in the spectroscopic sample and possible implications for disk clearing mechanisms.

\section{OBSERVATIONS AND ANALYSIS}

\subsection{Keck High-Resolution Echelle Spectrometer Observations and Analysis}

High-resolution optical spectra were obtained for 33 members of IC 348 using the High Resolution Echelle Spectrometer (HIRES; Vogt et al. 1994) on Keck I on 2006 November 28 and 30, and on 2007 November 29. Conditions were photometric on the first night with an average seeing of $\sim 0.6^{\prime \prime}$. Thin cirrus was present throughout the second night, which was mitigated by increasing integration times for faint sources. The third night was photometric with $\sim 1.0^{\prime \prime}$ seeing; however, summit fog resulted in the early closure of the telescope. HIRES was used with the red collimator and the C5 decker $\left(1.148^{\prime \prime} \times 7.0^{\prime \prime}\right)$, which has a projected slit width of 4 pixels and a spectral resolution of $\sim 33,000\left(8.8 \mathrm{~km} \mathrm{~s}^{-1}\right)$. The cross-disperser and echelle angles were set to approximately $0.884^{\circ}$ and $0.0^{\circ}$, respectively, providing nearly complete spectral coverage from about 4800 to $9200 \AA$. The selected wavelength range includes several gravity and temperature-sensitive photospheric features as well as permitted and forbidden transitions generally associated with accretion processes or chromospheric activity: $\mathrm{H} \beta, \mathrm{He}$ I $\lambda 5876$, [O I] $\lambda 6300, \mathrm{H} \alpha$, [S II] $\lambda \lambda 6717,6731$, and Ca II $\lambda \lambda 8498,8542$, and 8662. The red, green, and blue detectors were used in low gain mode, resulting in readout noise levels of 2.8, 3.1, and $3.1 \mathrm{e}^{-1}$, respectively. Internal quartz lamps were used for flat fielding and ThAr lamp spectra were used for wavelength calibration. The HIRES data were reduced using the MAuna Kea Echelle Extraction, makee, reduction script written by Tom Barlow. Although optimized for the spectral extraction of single, unresolved point sources, makee can be configured to extract individual spectra of close binaries. In addition to the object spectrum, the output of makee includes the $1 \sigma$ error spectrum, the sky and flat field spectra, and the count level spectrum. Given the range of apparent magnitudes for the observed sample, integration times varied from 15 to 45 min with typical signalto-noise ratios (S/Ns) of $\sim 30,50$, and 100 being achieved on the blue, green, and red chips, respectively. Signal-to-noise levels, however, could be significantly lower in the blue for heavily extincted sources.
The measured spectroscopic properties of the IC 348 membership sample observed with HIRES are given in Table 1. The columns tabulate the Luhman et al. (2003) identifier, assumed spectral type obtained from Luhman et al. (2003) or the literature, measured radial velocities and $v \sin i$ estimates with $1 \sigma$ uncertainties, and measured equivalent widths for $\mathrm{H} \beta$, $\mathrm{He}$ I $\lambda 5876$, $\mathrm{H} \alpha$, Li I $\lambda 6708$, and Ca II $\lambda \lambda 8498,8542,8662$. Measurement uncertainties for equivalent widths are $\sim 0.05 \AA$. Other emission lines (e.g. $\left[\mathrm{O}_{\mathrm{I}}\right] \lambda 6300$ ) found within the observed spectra are also tabulated. The final column of Table 1 gives the disk type associated with the star from the Lada et al. (2006) Spitzer IRAC SED analysis and whether the star is suspected of accreting, as determined by this investigation.

\subsection{SpeX Observations}

Moderate-resolution near-infrared spectra $(0.8-2.4 \mu \mathrm{m})$ were obtained for 39 members of IC 348 using SpeX on the NASA Infrared Telescope Facility (IRTF) on Mauna Kea. The observations were made on the nights of 2006 November 9-11 under photometric conditions and in $0.55^{\prime \prime}$ seeing. SpeX is a 0.8 $5.4 \mu \mathrm{m}$ cross-dispersed spectrograph and imager (Rayner et al. 2003). These spectra were obtained in the $0.8-2.4 \mu \mathrm{m}$ short cross-dispersed (SXD) mode using the $0.5^{\prime \prime}$ slit yielding a spectral resolution of $\sim 1500$. Integration times varied from 60 to $120 \mathrm{~s}$ for single coadds and multiple cycles were observed to yield total integration times of 20-40 min per source. The telescope was nodded along the slit in a " $\mathrm{a}-\mathrm{b}-\mathrm{b}-\mathrm{a}$ " pattern, allowing for sky subtraction. A0 V standard stars were observed for telluric corrections at air masses nearly identical to those of the observed program stars. Arc lamps and flat field calibration observations were made for each star to avoid potential calibration problems induced by flexure or changing slit position angles. The position angle of the slit was set to the parallactic angle for each series of program star, A0 standard star, and calibration spectra to minimize slit losses due to atmospheric refraction.

The SpeX spectra were reduced and extracted using SPEXTOOL, an IDL-based reduction package that provides for sky-subtraction, flat fielding, wavelength calibration, optimal extraction, and flux calibration (Cushing et al. 2004). The optimal extraction option was used for most single, uncomplicated sources. For the close double L8012, however, the slit was aligned along the axis of the binary to obtain spectra of each component of the system. To prevent confusion, the optimal extraction option was turned off and fixed extraction boundaries were established. For telluric corrections, an extension package available with SPEXTOOL, xtellcor, was used to interpolate over broad hydrogen absorption lines in the telluric standards using a technique developed by Vacca et al. (2003). In this method, a convolution kernel is generated from the spectra of the observed star and the A0 V standard star and then applied to a model spectrum of $\alpha$ Lyrae. After scaling the hydrogen lines of the model to those of the observed A0 V standard, the telluric spectrum is constructed by dividing the standard by the convolved and scaled Vega model. Residual wavelength shifts are then applied to each order, yielding the final telluric-corrected spectrum. Shown in Figures 2 and 3 are the merged 0.8-2.4 $\mu \mathrm{m}$ spectra for all primordial and weak or transition disk candidates in the IC 348 sample as well as select standards obtained from the IRTF spectral library (Rayner et al. 2008, in preparation). From the steeply rising slopes of the SEDs from 0.8 to $\sim 1.7 \mu \mathrm{m}$, it is apparent that most suffer significant extinction. Listed in Table 2 are the observed equivalent widths for emission features found in the near-infrared spectra of the suspected accretors in IC 348 
Table 1

Observed Spectroscopic Properties of IC 348 Members

\begin{tabular}{|c|c|c|c|c|c|c|c|c|c|c|c|c|c|}
\hline Identifier $^{\mathrm{a}}$ & $\mathrm{ST}^{\mathrm{b}}$ & $\begin{array}{l}A_{V}{ }^{\mathrm{c}} \\
(\mathrm{mag})\end{array}$ & $\begin{array}{c}V_{\mathrm{r}}^{\mathrm{d}} \\
\left(\mathrm{km} \mathrm{s}^{-1}\right)\end{array}$ & $\begin{array}{c}v \sin i^{\mathrm{d}} \\
\left(\mathrm{km} \mathrm{s}^{-1}\right)\end{array}$ & 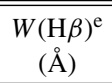 & $\begin{array}{c}W(\text { He I })^{\mathrm{e}} \\
\lambda 5876(\AA)\end{array}$ & $\begin{array}{c}(\mathrm{H} \alpha)^{\mathrm{e}} \\
(\AA)\end{array}$ & $\begin{array}{c}W(\text { Li I }) \\
\lambda 6708(\AA)\end{array}$ & $\begin{array}{l}W(\mathrm{Ca} \text { II })^{\mathrm{f}} \\
\lambda 8498(\AA)\end{array}$ & $\begin{array}{l}W(\mathrm{Ca} \text { II })^{\mathrm{f}} \\
\lambda 8542(\AA)\end{array}$ & $\begin{array}{l}W(\mathrm{Ca} \text { II })^{\mathrm{f}} \\
\lambda 8662(\AA)\end{array}$ & Other emission ${ }^{\mathrm{g}}$ & Comments ${ }^{\mathrm{h}}$ \\
\hline L8012A & $\mathrm{A}$ & 5.9 & $\ldots$ & $\ldots$ & +17.50 & & +11.46 & ... & . & . & . & & Non-excess? \\
\hline L8012B & A3: & 5.9 & $\ldots$ & $\ldots$ & +15.14 & +0.61 & -6.45 & $\ldots$ & $\ldots$ & $\ldots$ & $\ldots$ & 6300 & Accreting+primordial disk \\
\hline L38 & G0 & 2.1 & $12.37 \pm 1.28$ & $22.53 \pm 1.72$ & +1.85 & +0.08 & +2.39 & +0.18 & +0.84 & +2.32 & +1.89 & & Non-excess \\
\hline L31 & G1 & 11.8 & $13.28 \pm 1.63$ & $19.87 \pm 2.76$ & $\ldots$ & & -12.30 & +0.32 & -0.22 & -0.35 & -0.19 & 6300 & Accreting+primordial disk \\
\hline L20 & G1 & 1.8 & $25.17 \pm 0.98$ & $26.25 \pm 2.49$ & +1.66 & +0.05 & +2.04 & +0.21 & +1.08 & +2.44 & +1.75 & & Non-excess \\
\hline L6 & G3 & 3.3 & $11.37 \pm 1.67$ & $76.35 \pm 5.80$ & +2.04 & +0.11 & +0.70 & +0.21 & +0.81 & +2.04 & +1.59 & & Transition disk and rapid rotator \\
\hline L11 & G4 & 6.2 & $16.06 \pm 0.35$ & $17.84 \pm 2.79$ & +0.59 & +0.07 & -2.70 & +0.23 & -0.05 & -0.13 & +1.04 & & Non-excess \\
\hline L22 & G5 & 1.6 & $\mathrm{SB}(?)$ & $\mathrm{SB}(?)$ & +0.84 & $+0.03:$ & -0.94 & +0.28 & +0.66 & +1.74 & +1.29 & & Non-excess \\
\hline L16 & G6 & 2.5 & $14.10 \pm 1.72$ & $40.27 \pm 1.15$ & +1.38 & +0.09 & +1.24 & +0.27 & +0.81 & -0.07 & +1.47 & & Non-excess \\
\hline L5 & G8 & 4.4 & $2.90 \pm 1.43$ & $28.93 \pm 1.97$ & -1.74 & -0.45 & -21.93 & +0.35 & -0.67 & -1.01 & -0.58 & $6300,6678,6717,6731$ & Accreting+primordial disk \\
\hline L9 & G8 & 4.7 & $20.79 \pm 4.80$ & $\mathrm{SB}(?)$ & +1.52 & +0.12 & +1.39 & +0.25 & +0.79 & +1.97 & +1.54 & & Non-excess \\
\hline $\mathrm{L} 21$ & K0 & 5.4 & $17.01 \pm 0.84$ & $17.84 \pm 3.03$ & +1.26 & -0.10 & -4.48 & +0.44 & -0.28 & -0.48 & -0.30 & 6300 & Accreting+transition disk \\
\hline L44 & K0 & 1.3 & $14.89 \pm 1.04$ & $9.68 \pm 3.47$ & +0.19 & $+0.02:$ & -0.37 & +0.38 & -0.37 & -0.50 & -0.35 & & Non-excess \\
\hline L47 & K0 & 2.7 & $13.33 \pm 1.14$ & $16.53 \pm 2.14$ & +0.52 & +0.06 & +0.68 & +0.35 & -0.15 & -0.26 & -0.13 & & Non-excess \\
\hline L53 & K0 & 1.7 & $15.46 \pm 1.27$ & $19.65 \pm 2.46$ & +0.30 & +0.05 & -0.66 & +0.45 & -0.30 & -0.49 & -0.29 & & Non-excess \\
\hline L79 & K0 & 3.7 & .. & $\ldots$ & $\ldots$ & .. & $\ldots$ & $\ldots$ & $\ldots$ & $\ldots$ & $\ldots$ & & Non-excess, SpeX only \\
\hline L29 & K2 & 2.0 & $13.66 \pm 1.94$ & $42.24 \pm 2.35$ & +0.64 & +0.04 & +0.08 & +0.38 & -0.05 & -0.15 & -0.03 & & Non-Excess \\
\hline L59 & K2 & 3.7 & $13.92 \pm 1.09$ & $27.40 \pm 1.91$ & +0.64 & & -0.06 & +0.36 & -0.23 & -0.40 & -0.21 & & Non-excess \\
\hline L35 & K3 & 4.3 & $13.51 \pm 0.67$ & $\leqslant 9$ & +1.09 & +0.05 & -1.05 & +0.33 & +0.77 & +2.38 & +1.77 & & Non-excess \\
\hline L23 & K3 & 6.1 & $\ldots$ & $\ldots$ & & $\ldots$ & $\ldots$ & $\ldots$ & $\ldots$ & $\ldots$ & $\ldots$ & & Non-excess, SpeX only \\
\hline L56 & K4 & 2.4 & $14.07 \pm 0.87$ & $11.56 \pm 5.23$ & +0.59 & +0.07 & +0.35 & +0.44 & -0.10 & -0.17 & -0.07 & & Non-excess \\
\hline L39 & K4 & 6.1 & $\ldots$ & $\ldots$ & & $\ldots$ & $\ldots$ & $\ldots$ & $\ldots$ & $\ldots$ & $\ldots$ & & Non-excess, SpeX only \\
\hline L50 & K4 & 7.7 & & 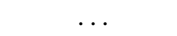 & & & $\ldots$ & . & $\ldots$ & .. & & & Non-excess, SpeX only \\
\hline L45 & K5 & 2.0 & $11.48 \pm 1.97$ & $40.04 \pm 1.96$ & +0.32 & +0.05 & -0.47 & +0.52 & -0.13 & -0.32 & -0.09 & & Non-excess \\
\hline
\end{tabular}


Table 1

(Continued)

\begin{tabular}{|c|c|c|c|c|c|c|c|c|c|c|c|c|c|}
\hline Identifier $^{\mathrm{a}}$ & $\mathrm{ST}^{\mathrm{b}}$ & $\begin{array}{c}A_{V}{ }^{\mathrm{c}} \\
(\mathrm{mag})\end{array}$ & $\begin{array}{c}V_{\mathrm{r}}^{\mathrm{d}} \\
\left(\mathrm{km} \mathrm{s}^{-1}\right)\end{array}$ & $\begin{array}{c}v \sin i^{\mathrm{d}} \\
\left(\mathrm{km} \mathrm{s}^{-1}\right)\end{array}$ & $\begin{array}{c}W(\mathrm{H} \beta)^{\mathrm{e}} \\
(\AA)\end{array}$ & $\begin{array}{c}W\left(\mathrm{He} \mathrm{I}^{\mathrm{e}}\right. \\
\lambda 5876(\AA)\end{array}$ & $\begin{array}{c}W(\mathrm{H} \alpha)^{\mathrm{e}} \\
(\AA)\end{array}$ & $\begin{array}{c}W(\mathrm{Li} \mathrm{I}) \\
\lambda 6708(\AA) \\
\end{array}$ & $\begin{array}{l}W(\mathrm{Ca} \text { II })^{\mathrm{f}} \\
\lambda 8498(\AA)\end{array}$ & $\begin{array}{l}W(\mathrm{Ca} \text { II })^{\mathrm{f}} \\
\lambda 8542(\AA)\end{array}$ & $\begin{array}{l}W(\mathrm{Ca} \text { II })^{\mathrm{f}} \\
\lambda 8662(\AA)\end{array}$ & Other emission $^{\mathrm{g}}$ & Comments $^{\mathrm{h}}$ \\
\hline L48 & $\mathrm{K} 5.5$ & 2.6 & $12.47 \pm 0.93$ & $15.60 \pm 2.25$ & -0.13 & -0.02 & -1.56 & +0.54 & -0.49 & -0.70 & -0.41 & & Non-excess \\
\hline L36 & K6 & 2.9 & $16.47 \pm 0.69$ & $16.73 \pm 4.16$ & -2.84 & -0.13 & -8.03 & +0.49 & -0.68 & -0.94 & -0.61 & 6300,6731 & Accreting+primordial disk \\
\hline L37 & K6 & 2.2 & $23.55 \pm 1.25$ & $\leqslant 9$ & -3.36 & -0.24 & -5.84 & +0.48 & -0.39 & -0.55 & -0.32 & 6300,6731 & Accreting+primordial disk \\
\hline L10363 & K6 & 4.0 & & & & & & & & & & & Non-excess, SpeX only \\
\hline L8024 & K6.5 & 2.2 & $10.24 \pm 2.01$ & $74.24 \pm 6.05$ & +1.27 & +0.08 & +0.70 & +0.21 & +0.79 & +2.01 & +1.55 & & Transition disk, rapid rotator \\
\hline L66 & K6.5 & 1.7 & $14.44 \pm 0.78$ & $14.68 \pm 3.85$ & -0.30 & -0.03 & -1.10 & +0.59 & -0.36 & -0.51 & -0.31 & & Non-excess \\
\hline L41 & K7 & 3.2 & $11.44 \pm 1.04$ & $13.72 \pm 3.73$ & -25.85 & -0.97 & -51.12 & +0.49 & -2.39 & -2.25 & -2.41 & 6300,6678 & Accreting+primordial disk \\
\hline L32 & K7 & 3.7 & $13.41 \pm 1.21$ & $13.50 \pm 5.24$ & -9.66 & -1.24 & -34.41 & +0.44 & -0.66 & -0.95 & -0.57 & 6300,6678 & Accreting+primordial disk \\
\hline L26 & K7 & 6.9 & $13.30 \pm 1.33$ & $15.20 \pm 2.45$ & -7.71 & -0.39 & -31.50 & +0.45 & -4.34 & -4.86 & -3.73 & 6300 & Accreting+primordial disk \\
\hline L82 & K7 & 2.5 & $15.05 \pm 1.12$ & $15.43 \pm 5.47$ & -0.83 & +0.07 & -0.93 & +0.55 & -0.26 & -0.42 & -0.20 & & Non-excess \\
\hline L61 & K8 & 3.5 & $6.23 \pm 1.68$ & $25.31 \pm 4.63$ & -4.87 & -0.49 & -48.00 & +0.44 & -0.25 & -0.35 & -0.18 & 6300,6731 & Accreting+primordial disk \\
\hline L40 & K8 & 4.0 & $11.19 \pm 1.26$ & $16.78 \pm 4.36$ & -50.94 & -3.25 & -114.28 & +0.47 & -4.21 & -5.98 & -4.96 & 6300,6678 & Accreting+primordial disk \\
\hline L65 & M0 & 1.6 & $11.20 \pm 0.90$ & $14.05 \pm 4.44$ & -0.69 & +0.01 & -0.96 & +0.52 & -0.44 & -0.67 & -0.46 & & Transition disk \\
\hline L9024 & M0 & 3.5 & $14.66 \pm 1.30$ & $16.67 \pm 3.57$ & -2.17 & -0.55 & -10.42 & +0.57 & -0.48 & -0.65 & -0.44 & 6300 & Accreting+primordial disk \\
\hline L9060 & M0 & 3.3 & $\ldots$ & $\ldots$ & & $\ldots$ & $\ldots$ & $\ldots$ & $\ldots$ & $\ldots$ & $\ldots$ & & primordial disk, SpeX only \\
\hline L1937 & M0 & 3.3 & & & & 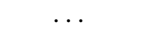 & & & & & & & Non-excess, SpeX only \\
\hline L67 & M0.75 & 1.20 & $15.09 \pm 0.75$ & $13.59 \pm 3.96$ & & 0.86 & 28 & +0.51 & 0.46 & -0.64 & -0 & 6300,6678 & Accreting+transition disk \\
\hline
\end{tabular}

Notes.

a Identifier from Luhman et al. (2003) cluster census.

${ }^{\mathrm{b}}$ Spectral type from the literature.

${ }^{c}$ Extinction estimates derived from color excesses, assuming the normal reddening relationship: $A_{v}=2.43 E\left(V-I_{\mathrm{C}}\right)$

${ }^{\mathrm{d}}$ Radial velocities and $v \sin i$ estimates derived from cross-correlation analysis of HIRES observations. SB: possible spectroscopic binary.

e Negative values indicate emission.

${ }_{\mathrm{f}}^{\mathrm{f}}$ Negative values indicate emission. If core emission reversal is present, equivalent widths are measured from the base of the Ca II absorption profile.

[O I] $\lambda 6300, \mathrm{He}$ I $\lambda 6678,[\mathrm{~S}$ II] $\lambda \lambda 6617,6731$

${ }^{\mathrm{h}}$ Disk classification based upon the SED slope through the IRAC channels, from Lada et al. (2006). 


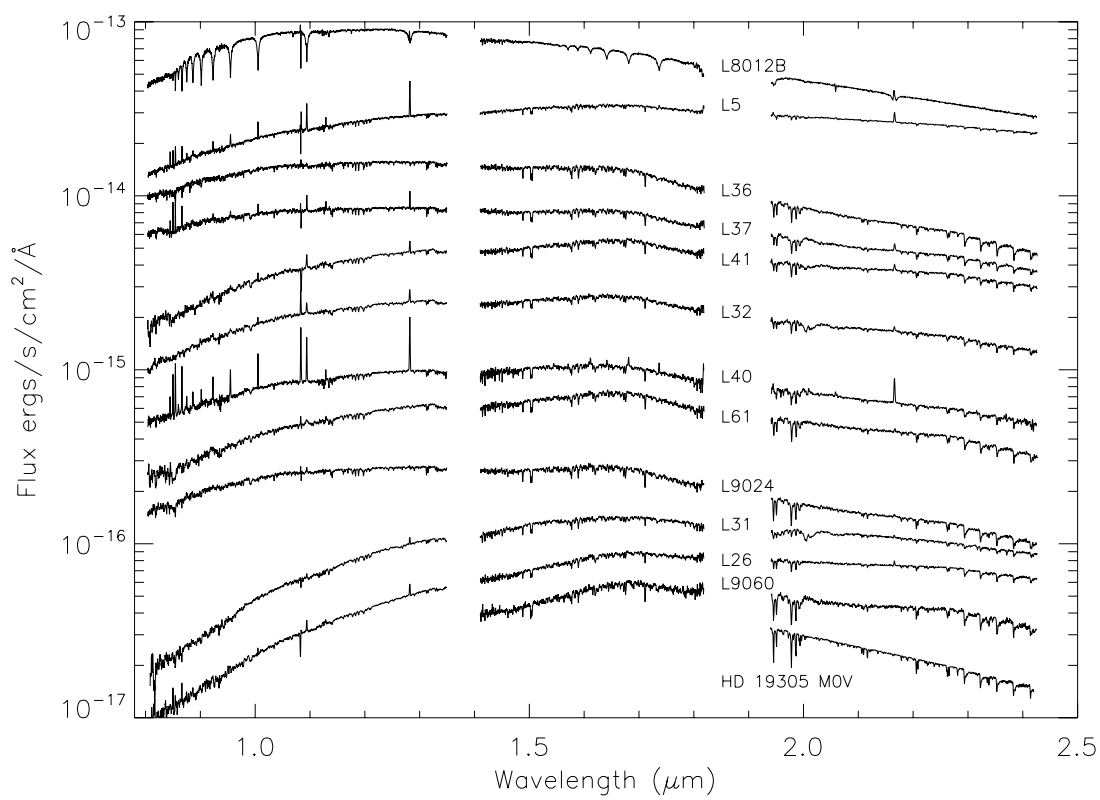

Figure 2. Moderate-resolution, near-infrared spectra $(0.8-2.4 \mu \mathrm{m})$ obtained using SpeX on IRTF for the 12 primordial disk candidates in the IC 348 membership sample. High extinction for all of the sources can be inferred from the steeply rising SEDs from $\sim 0.8$ to $1.5 \mu \mathrm{m}$. All of the primordial disk candidates with the exception of the M0-type L9060 exhibit $\mathrm{H}$ I $(\mathrm{Pa} \gamma, \mathrm{Pa} \beta, \mathrm{Br} \gamma)$ or He I $(1.083,2.06 \mu \mathrm{m})$ emission in the near-infrared. The $K$-band spectrum of the M0 V standard HD 19305 is also shown, clearly demonstrating the $K$-band excesses present among all primordial disk-bearing stars. The strongest accretors (L5, L40, L37) also exhibit significant Ca II emission, readily apparent even at the low dispersion and reduced "blue" sensitivity of SpeX.

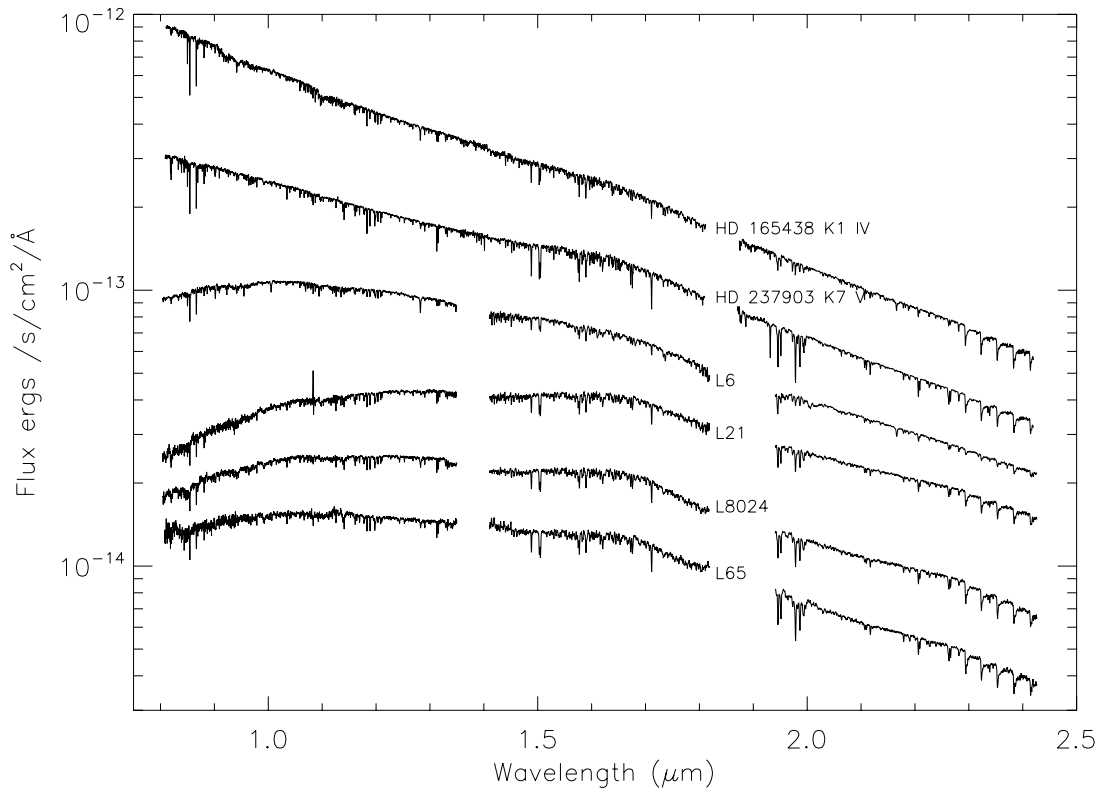

Figure 3. Near-infrared spectra (0.8-2.4 $\mu \mathrm{m})$ obtained using SpeX on IRTF for the four weak or transition disk candidates in the IC 348 membership sample. Two standards (K1 IV and K7 V types) from the IRTF spectral library are shown for comparison. All transition disk candidates with the exception of the G3-type L6 exhibit slight $K$-band excesses similar to those observed among the primordial disk candidates.

including Ca II $\lambda \lambda 8498,8542,8662, \mathrm{He}$ I $\lambda 10830, \mathrm{~Pa} \beta$, and $\mathrm{Br} \gamma$. Also listed are the line luminosities for $\mathrm{Pa} \beta$ and $\mathrm{Br} \gamma$ if found in emission or derived upper limits (see Section 4.3).

\section{THE IC 348 MEMBERSHIP SAMPLE}

The Luhman et al. (2003) census of IC 348 incorporates several earlier surveys of the cluster including those of Herbig (1954, 1998), Lada \& Lada (1995), Luhman et al. (1998), Luhman (1999), and Muench et al. (2003). Additional members were identified within a $42^{\prime} \times 28^{\prime}$ region using the extinctioncorrected, $(I-K, H)$ and $(I-Z, H)$ color-magnitude diagrams
(CMDs) and the theoretical isochrones of Baraffe et al. (1998). Established members of the cluster from earlier surveys were observed to lie above the $10 \mathrm{Myr}$ isochrone of Baraffe et al. (1998), which was subsequently used to distinguish all candidate members from field stars. Spectral types were determined for 268 of these stars using low-resolution, optical spectra. In total, 288 stars were identified as likely members, 23 of which are later than M6 and probable brown dwarfs. From a Spitzer survey of the region, Muench et al. (2007) added 42 class II sources and 20 embedded class 0 and I sources to the population of IC 348, but these are predominantly later type stars ( $>\mathrm{M} 3)$. This program specifically targets spectral types from M0 to G0, a critical mass 
Table 2

Near-Infrared Spectroscopic Properties of Suspected Accreting IC 348 Members

\begin{tabular}{lcccccccc}
\hline \hline Identifier $^{\mathrm{a}}$ & $\begin{array}{c}W(\mathrm{Ca} \mathrm{II})^{\mathrm{b}} \\
\lambda 8498(\AA)\end{array}$ & $\begin{array}{c}W(\mathrm{Ca} \mathrm{II})^{\mathrm{b}} \\
\lambda 8542(\AA)\end{array}$ & $\begin{array}{c}W(\mathrm{Ca} \mathrm{II})^{\mathrm{b}} \\
\lambda 8662(\AA)\end{array}$ & $\begin{array}{c}W(\mathrm{He} \mathrm{I})^{\mathrm{b}} \\
\lambda 10830(\AA)\end{array}$ & $\begin{array}{c}W(\mathrm{~Pa} \beta)^{\mathrm{b}} \\
(\AA)\end{array}$ & $\begin{array}{c}W(\mathrm{Br} \gamma)^{\mathrm{b}} \\
(\AA)\end{array}$ & $\begin{array}{c}\log L(\mathrm{~Pa} \beta)^{\mathrm{c}} \\
\operatorname{erg~s}^{-1}\end{array}$ & $\begin{array}{c}\log L(\mathrm{Br} \gamma)^{\mathrm{c}} \\
\operatorname{erg~s}^{-1}\end{array}$ \\
\hline L8012B & $\ldots$ & $\ldots$ & $\ldots$ & +1.50 & -1.18 & -4.86 & 30.16 & 30.05 \\
L5 & -2.60 & -4.60 & -3.05 & -0.87 & -9.13 & -3.69 & 31.02 & 30.41 \\
L31 & $\ldots$ & $\ldots$ & $\ldots$ & +0.31 & -3.69 & -3.17 & 30.92 & 30.09 \\
L21 & $\ldots$ & $\ldots$ & $\ldots$ & -2.05 & -0.96 & -0.69 & 29.95 & 29.41 \\
L40 & -7.99 & -11.56 & -10.10 & -14.14 & -16.60 & -7.80 & 30.47 & 29.68 \\
L37 & -4.57 & -5.55 & -4.43 & +1.08 & -3.25 & -2.06 & 29.72 & 29.19 \\
L36 & $\ldots$ & $\ldots$ & $\ldots$ & -1.11 & $\ldots$ & $\ldots$ & $<28.86$ & $<27.94$ \\
L41 & $-1.00:$ & $-2.35:$ & $-1.38:$ & +3.32 & -3.25 & -2.00 & 29.50 & 28.91 \\
L32 & $-0.93:$ & $-1.58:$ & $-0.97:$ & -4.97 & -3.37 & -1.44 & 29.88 & 28.89 \\
L26 & -4.47 & -4.48 & -6.11 & +2.15 & -2.93 & -1.03 & 30.29 & 29.26 \\
L61 & $\ldots$ & $\ldots$ & $\ldots$ & -1.78 & $\ldots$ & -0.96 & $<28.97$ & 28.47 \\
L9024 & $\ldots$ & $\ldots$ & $\ldots$ & -0.70 & -1.34 & $\ldots$ & 29.34 & 28.50 \\
L9060 & $\ldots$ & $\ldots$ & $\ldots$ & $\ldots$ & $\ldots$ & $\ldots$ & $<28.20$ & $<27.88$ \\
\hline
\end{tabular}

Notes.

a Identifier from Luhman et al. (2003) cluster census.

b Negative values indicate emission.

${ }^{\mathrm{c}}$ Determined from the flux calibrated spectra.

range from $\sim 0.5$ to $2.0 M_{\odot}$ that includes young solar analogs. Beyond the relevance to our own Sun and solar system, the reasonably bright apparent magnitudes of this sample permits the acquisition of high-resolution, optical spectra in reasonable integration times, despite extinctions as high as $A_{V} \sim 12.3 \mathrm{mag}$. The Luhman et al. (2003) census of IC 348 identifies 45 stars spanning the proposed range in spectral type, all of which have 3.6-8.0 $\mu \mathrm{m}$ Spitzer IRAC photometry from Lada et al. (2006).

HIRES and SpeX observations were obtained for 32 of 45 M0-G0 type stars in IC 348, including 11 primordial disk candidates, 5 weak or transition disk systems, and 16 noninfrared excess stars. An additional seven non-excess stars and one primordial disk candidate were observed using SpeX only, bringing the total number of IC 348 members examined to 40, or $\sim 87 \%$ of the cluster population within this range of spectral types. One weak or transition disk object included here, L67, has a spectral type of M0.75, somewhat later than the other cluster members in the survey. Spectral types assigned by Luhman et al. (2003 and references therein) were confirmed using the moderate resolution $J$ - and $H$-band spectra obtained with SpeX. Beyond $\sim 2 \mu \mathrm{m}$, continuum excess emission from hot, micronsized dust grains fills in photospheric features, limiting the effectiveness of $K$-band spectral classification for stars hosting optically thick inner disks. The IRTF spectral atlas (Rayner et al. 2008 , in preparation), the $J$-band spectral catalog of Wallace et al. (2000) and the $H$-band catalog of Meyer et al. (1998) are used for comparison with the IC 348 membership sample. Numerous $J$-band transitions of neutral metals were examined for classification purposes, including $\mathrm{Al}$ I 1.312 and $1.315 \mu \mathrm{m}$, Mg I $1.183 \mu \mathrm{m}$, and Mn I $1.289 \mu \mathrm{m}$. The temperature sensitive spectral indices of Wallace et al. (2000) for main-sequence stars and giants were adopted to bracket the effective temperatures of the IC 348 members. The $H$-band transitions used for spectral classification included $\mathrm{Mg}$ I 1.576 and $1.711 \mu \mathrm{m}, \mathrm{OH}$ $1.689 \mu \mathrm{m}$, Al I $1.674 \mu \mathrm{m}$, and Si I 1.596 and $1.668 \mu \mathrm{m}$. The temperature indices of Meyer et al. (1998) for dwarfs and giants were also evaluated for stars later than $\sim \mathrm{K} 3$ to ensure temperatures fell within expectations for their given spectral type. The uncertainties associated with the temperature indices are \pm 2 spectral subclasses or $\pm 300 \mathrm{~K}$. Shown in the Appendix are the SpeX $J$-band (1.15-1.35 $\mu \mathrm{m}), H$-band $(1.47-1.75 \mu \mathrm{m})$, and $K$-band (2.0-2.42 $\mu \mathrm{m})$ spectra for 36 of the IC 348 members observed.

Few changes were made to the spectral types assigned by the earlier surveys. One star, however, (L8012) was classified as a G0 spectral type and is in fact a much earlier type star as well as a close binary. Both components of L8012 appear to be early-to-mid A spectral types and are evidently deeply embedded with an estimated $A_{V} \sim 6$ mag. The Spitzer derived SED of L8012 reveals a significant mid-infrared excess extending from $4.5 \mu \mathrm{m}$ (IRAC ch 2) to $24 \mu \mathrm{m}$ (MIPS ch 1). The pair are separated by just over an arcsecond and would thus be unresolved in the Spitzer IRAC and MIPS-band photometry. The primary or more luminous component of the pair exhibits an unexceptional spectrum at optical and near-infrared wavelengths. All $\mathrm{H}$ I and He I lines are seen in absorption. The secondary component, however, exhibits $\mathrm{H} \alpha$ emission rising well above the continuum although not completely filling the wings of the photospheric absorption line profile. Herbig (1998) does not report $\mathrm{H} \alpha$ emission from this star, which would have been readily detectable in the Canada-France-Hawaii Telescope multi-object spectrograph spectrum or the slitless grism observations if emission were present at the time of observation. $\mathrm{Pa} \beta$ appears with emission reversal, but does not reach the continuum level whereas $\mathrm{Br} \gamma$ clearly rises above the continuum. Also present in emission is the He $\mathrm{I} \lambda 10830$ line which exhibits a deep red-shifted absorption feature. This inverse P Cygni profile is interpreted as evidence for magnetospheric accretion flows, observed through the infalling gas and the accretion shock. The evidence for gaseous accretion in the spectrum of the secondary suggests that the dust emission detected by Spitzer is associated with this component of the binary.

\subsection{The Color-Magnitude and Color-Color Diagrams of the Stellar Sample}

Shown in Figure 4 is the extinction-corrected $\left(V-I_{\mathrm{C}}, I_{\mathrm{C}}\right)$ CMD for the 40 stars comprising the spectroscopic sample of IC 348. To correct the individual stars for extinction effects, we use spectral types to determine $A_{V}$ values from $V-I_{\mathrm{C}}$ color excesses, assuming the normal reddening relationship, $A_{V}=3.08 E(B-V)=2.43 E\left(V-I_{\mathrm{C}}\right) . A_{J}$ and $A_{V}$ values tabulated by Luhman et al. (2003) and Lada et al. (2006) 


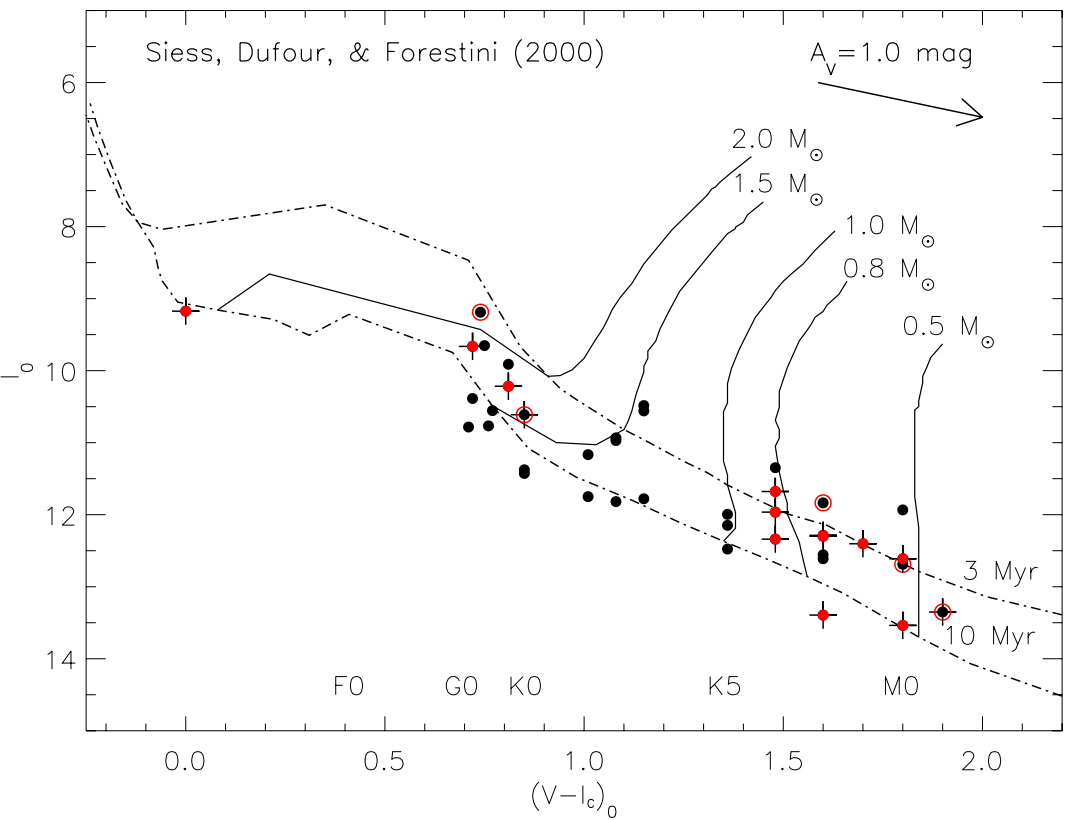

Figure 4. The extinction-corrected $\left(V-I_{\mathrm{C}}, I_{\mathrm{C}}\right) \mathrm{CMD}$ for the 40 stars comprising the spectroscopic sample of IC 348 . Stars have been individually corrected for extinction using their assigned spectral types and $V-I_{\mathrm{C}}$ color excesses to determine $A_{V}$ values. When $V$-band photometry is not available, $A_{J}$ and $A_{V}$ values tabulated by Luhman et al. (2003) and Lada et al. (2006) are adopted for the sample. Superposed in the figure are the theoretical pre-main-sequence isochrones and evolutionary tracks of Siess et al. (2000), plotted assuming a distance of $320 \mathrm{pc}$ to the cluster. Primordial disk-bearing stars (as inferred from the IRAC SED slope parameter analysis of Lada et al. 2006) are represented as solid red circles, weak or transition disk candidates are shown as solid black points encircled in red, non-excess stars as solid black circles, and suspected accretors from this investigation are marked by crosses. We infer from the models of Siess et al. (2000) that the spectroscopic sample of IC 348 ranges from masses of $\sim 2.0-0.5 M_{\odot}$.

(A color version of this figure is available in the online journal)

were adopted when $V$-band photometry was not available. Superposed in Figure 4 are several theoretical pre-main-sequence isochrones and evolutionary tracks from the models of Siess et al. (2000), plotted assuming a distance of $320 \mathrm{pc}$. We infer from the models that the spectroscopic sample of IC 348 ranges from $\sim 0.5$ to $2.0 M_{\odot}$ and in age from $\sim 1$ to $10 \mathrm{Myr}$. The significant age dispersion suggested by the broadened pre-main sequence is partially attributable to observational error, unresolved binaries, neutral extinction induced by edge-on disks, or intrinsic source variability. The tendency for the theoretical isochrones to systematically predict older ages for more massive stars $\left(>1 M_{\odot}\right.$ ) is well documented (Hillenbrand 1997; Sung et al. 2004) and affects all sets of pre-main-sequence models. It is not further addressed here, but should be considered when interpreting the inferred age dispersion and the star-formation history of the cluster. Mass estimates for the observed sample suffer predominantly from extinction errors given that the reddening vector is nearly perpendicular to the convective tracks. Other uncertainties include photometric errors and the usage of mainsequence colors when dereddening the stars. From the CMD, we conclude that all stars in the spectroscopic sample have colors and luminosities consistent with cluster membership.

Shown in Figure 5 is the $K_{\mathrm{S}}-4.5 \mu \mathrm{m}, J-H$ colorcolor diagram for the 40 stars in the spectroscopic sample plotted using Spitzer IRAC $4.5 \mu \mathrm{m}$ photometry from Lada et al. (2006) and $J H K_{S}$ photometry from the Two Micron All Sky Survey (2MASS) point source catalog. Symbols used in the figure distinguish stars on the basis of their infrared excesses as determined by the IRAC SED slope parameter from Lada et al. (2006). All of the non-disk candidates lie along the reddening vector above the near-vertical trunk representing normal mainsequence colors. The primordial disk candidates and three weak or transition disk systems (L21, L6, and L67) are well separated from the non-excess stars, lying on the extreme redward edge of the M-dwarf locus. The separation between disk-bearing and non-excess stars is quite striking, implying that the transition disk systems must evolve rapidly through the intervening region of the color-color diagram. All of the observed stars have infrared colors consistent with cluster membership, but it is readily apparent that most suffer significant extinction. Shown in Figure 6 is the logarithm of the ratio of 8.0 and $4.5 \mu \mathrm{m}$ fluxes plotted as a function of intrinsic $J-H$ color, a tracer of stellar photospheric emission for the observed IC 348 sample. All primordial disk candidates and three of five weak or transition disk candidates appear well separated from the non-excess stars in this flux-ratio, $(J-H)_{0}$ color diagram.

\subsection{Lithium Abundances}

To examine whether the membership sample is young, we have used $\mathrm{Li}$ I $\lambda 6707.8$ line strengths as a youth discriminant. Lithium is destroyed at the high temperatures $(>2.5 \mathrm{MK})$ expected within the cores of these deeply convective pre-mainsequence stars. Consequently, surface lithium abundance serves as an age index for these stars. Shown in Figure 7 is $\mathrm{W}(\lambda 6707.8)$ plotted as a function of effective temperature $\left(T_{\mathrm{e}}\right)$ for the 32 stars in the sample with high-resolution, optical spectroscopy available. $T_{\mathrm{e}}$ values were derived from the tabulation of mainsequence properties by Kenyon \& Hartmann (1995). At the resolution of HIRES, $\mathrm{Li}$ I $\lambda 6707.8$ is well separated from all nearby $\mathrm{Fe}$ I and $\mathrm{Ca}$ I lines with the exception of the weak $\mathrm{Fe}$ I $\lambda 6707.45$ feature. No corrections for this blended feature or for possible optical veiling effects have been applied to the $W(\lambda 6708)$ values presented here. Also shown in Figure 7 are the $\mathrm{W}(\lambda 6707.8)$ measurements of $126 \mathrm{~F}-, \mathrm{G}-$, and K-type members of the $\sim 100 \mathrm{Myr}$ old Pleiades cluster taken from Soderblom 


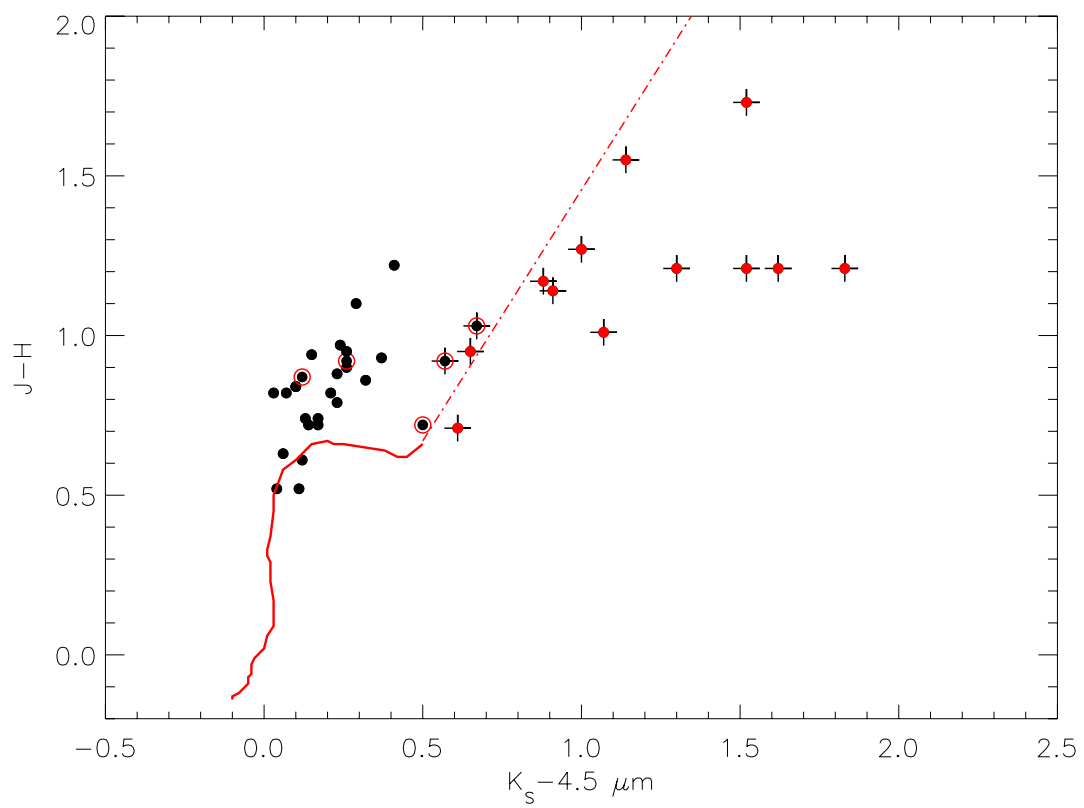

Figure 5. The $\left(K_{\mathrm{S}}-4.5 \mu \mathrm{m}, J-H\right)$ color-color diagram for the IC 348 membership sample. The curved solid red line represents the intrinsic colors of normal main-sequence stars, derived from the $J-H$ colors of Tokunaga (2000) and interpolation of the $K_{\mathrm{S}}-4.5 \mu \mathrm{m}$ main-sequence colors of Lada et al. (2006). The red dot-dashed line extending above this curve delineates the approximate reddening boundary for dwarfs, the slope of which was derived using $M$-band extinction curve data from Martin \& Whittet (1990). Symbols are as in Figure 4. The primordial disk candidates are well separated from the non-excess stars as are the transition disk systems, L21, L6, and L67. Most of the cluster members suffer significant extinction as evidenced by their displacement from the dwarf locus.

(A color version of this figure is available in the online journal)

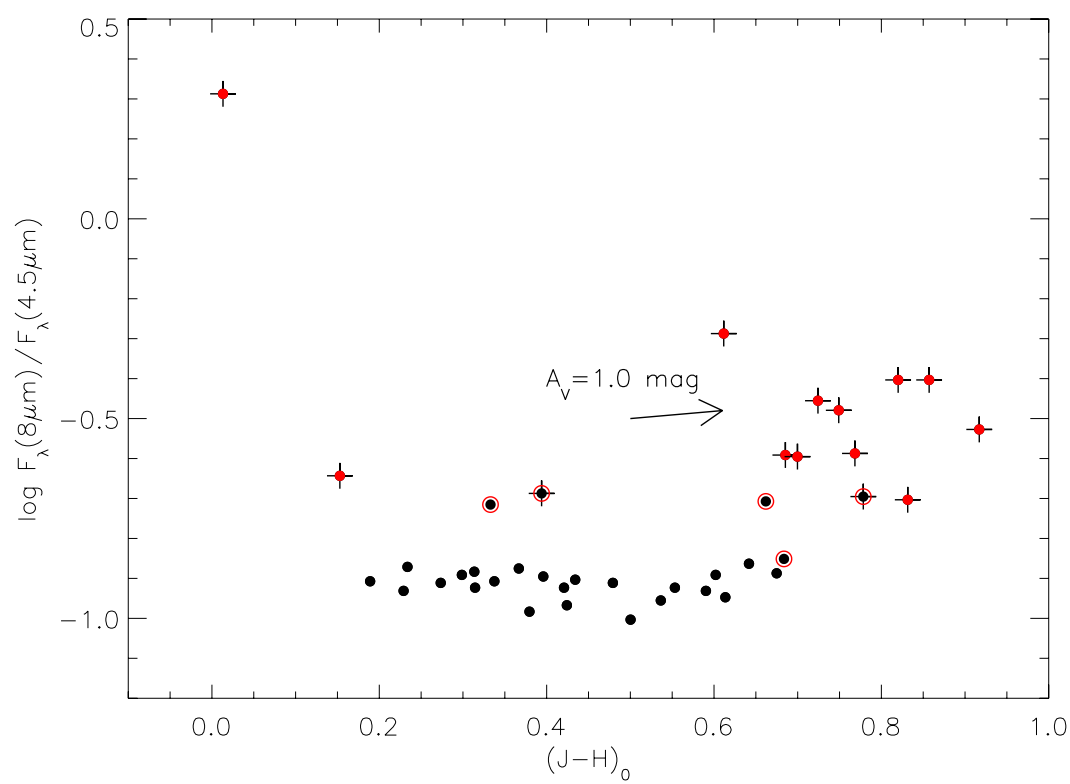

Figure 6. The logarithm of the Spitzer IRAC 8.0 to $4.5 \mu \mathrm{m}$ flux ratio plotted as a function of $J-H$ color (a tracer of photospheric emission) for all members of the IC 348 spectroscopic sample. Symbols used are as in Figure 4. The primordial disk candidates lie well separated from the non-excess stars as do three weak or transition disk candidates. The star exhibiting the most significant flux ratio is the early-type binary, L8012, which is unresolved by the Spitzer photometry.

(A color version of this figure is available in the online journal)

et al. (1993). The steep decline in $\mathrm{W}(\lambda 6707.8)$ for Pleiades members at $T_{\mathrm{e}}<4000 \mathrm{~K}$ is attributable to the destruction of lithium in these predominantly convective stars. With the one exception of L8024, all of the IC 348 members lie above the sequence of Pleiades stars (an upper limit for the early-type star L8012B is given). L8024 is a weak or transition disk system and a suspected rapid rotator that exhibits very broad photospheric absorption lines. From the lack of veiling and the presence of weak $\mathrm{H} \alpha$ emission, we conclude that L8024 is not accreting. The star is also a visual double with a companion (L9024) lying $4^{\prime \prime}$ to the east. L9024 is a primordial disk candidate and accretor and retains a lithium abundance consistent with that of other IC 348 members. If these stars form a true binary (separation distance $\sim 1300 \mathrm{AU}$ ), the disparate lithium abundances would serve as a less dramatic example of the phenomenon observed in HIP 112312AB, the M4 type $\beta$ Pic binary (Song et al. 2002).

\subsection{Radial and Projected Rotational Velocity Determinations}

Radial velocities were determined for all stars listed in Table 1 observed with HIRES using the fxcor task available 


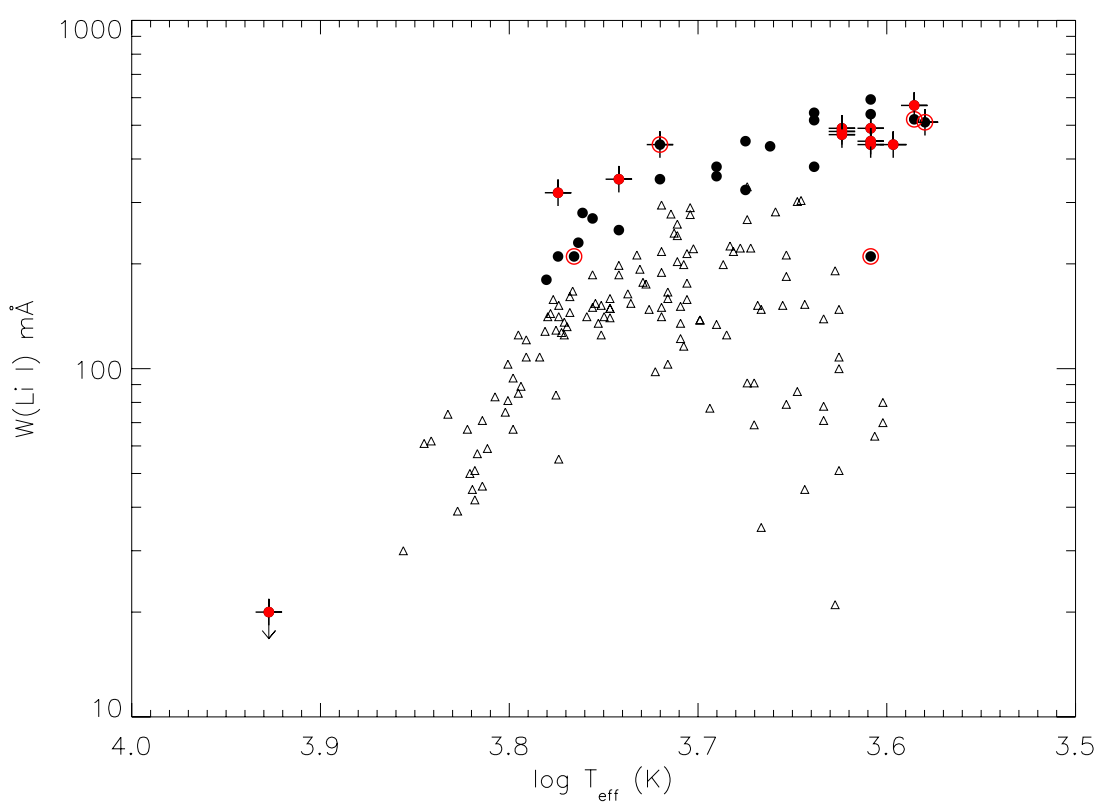

Figure 7. $\mathrm{W}(\lambda 6708)$ for the 31 IC 348 members observed with HIRES plotted as a function of $\log T_{\mathrm{e}}$. Symbols used are as in Figure 4 with the addition of open triangles that represent Li I measurements for 126 Pleiades F-, G-, and K-type members obtained from Soderblom et al. (1993). With the exception of L8024, all IC 348 members lie above the sequence defined by the Pleiades dwarfs. L8024 is a weak or transition disk system and a suspected rapid rotator with broad photospheric absorption lines. The single upper limit shown is for the early-type IC 348 member, L8012B.

(A color version of this figure is available in the online journal)

in the $r v$ package of IRAF. fxcor performs Fourier crosscorrelation analysis to determine the difference in radial velocities between the observed star and a template star of known radial velocity. Several spectral regions free of emission lines or strong telluric features were used in the cross-correlation analysis resulting in radial velocities accurate to $0.7-2 \mathrm{~km} \mathrm{~s}^{-1}$. The final radial velocities presented in Table 1 have been corrected for diurnal and lunar velocities using the IRAF task rvcorrect. Slowly rotating spectral standards with well-established radial velocities were observed on all nights to serve as stellar templates. Uncertainties in the tabulated radial velocities represent the standard deviations of the multiple radial velocity estimates (10-20 per star) generated from the various echelle orders used in the cross-correlation analysis. Radial velocities were not determined for either component of L8012 given the lack of narrow photospheric absorption features, or for L22, which exhibits a broadened cross-correlation maximum having two peaks. It is possible that this star is a spectroscopic binary, a supposition that is supported by the broadened photospheric absorption lines present and the appearance of two minima within some of these.

Shown in Figure 8 is the histogram of measured heliocentric radial velocities for the remaining cluster members having HIRES observations. The median value of $13.5 \mathrm{~km} \mathrm{~s}^{-1}$ is consistent with that of an expanding shell of gas associated with IC 348, 13-14 $\mathrm{km} \mathrm{s}^{-1}$ (Snow et al. 1994), and that of HD 281159, the most luminous cluster member, $\sim 14.0 \mathrm{~km} \mathrm{~s}^{-1}$ (Snow et al. 1994). Nordhagen et al. (2006) present radial velocities for about 30 cluster members and find a mean value of $15.9 \pm 0.8 \mathrm{~km} \mathrm{~s}^{-1}$, which is also consistent with the results presented here. Comparing velocities of the 11 stars in common between these two surveys, we find relative agreement (within errors) for most, but significant discrepancies for L37, L61, and L65. It is possible that these stars are spectroscopic binaries, but additional high-resolution observations are needed to resolve this issue. The five outliers of the radial velocity distribution shown in Figure 8 are L20, L9, L37, L5, and L61, the latter three of

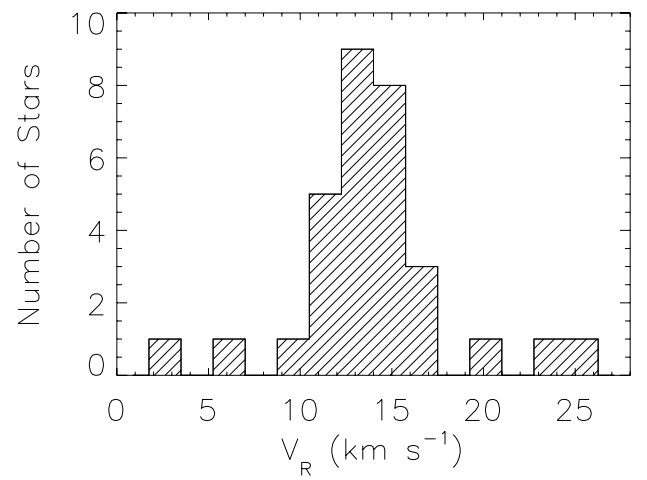

Figure 8. Histogram of heliocentric radial velocities for the IC 348 sample observed using HIRES. The median value of $13.5 \mathrm{~km} \mathrm{~s}^{-1}$ is consistent with published radial velocities for the expanding supernova remnant associated with the cluster and with the B5 member, HD 281159. The five apparent outliers in the distribution are L20, L9, L37, L5, and L61. The measured radial velocities for all stars are consistent with cluster membership.

which are primordial disk candidates. Considering just the subsample of accreting IC 348 members, we find the median radial velocity to be $13.4 \mathrm{~km} \mathrm{~s}^{-1}$, essentially identical to that of the population as a whole. In conclusion, all of the suspected IC 348 stars have radial velocities consistent with cluster membership.

Projected rotational velocities $(v \sin i)$ for all IC $348 \mathrm{mem}-$ bers observed with HIRES were determined by Fourier crosscorrelation analysis using a slowly rotating standard star as a template. Gaussian profiles were then fitted to the crosscorrelation peak to measure the full width at half-maximum (FWHM), which was then applied to an empirical relationship between the FWHM and $v \sin i$ for a given order of HIRES. These FWHM $-v \sin i$ relationships were derived by convolving the one dimensional convolution kernel for a specified $v \sin i$ value to the spectrum of a slowly rotating standard. The artificially spun up spectrum assumes constant limb darkening across the disk with a limb darkening coefficient of 0.6 , typical of stellar 
photospheric lines. Uncertainties for $v \sin i$ given in Table 1 reflect the standard deviation of values from all orders examined. Typically 7 HIRES orders free of telluric features and strong emission lines were used for the $v \sin i$ estimates. Upper limits are assigned for several stars with projected rotational velocities that fall within errors of the velocity resolution, $\sim 9 \mathrm{~km} \mathrm{~s}^{-1}$. Measured $v \sin i$ values were compared with those of Nordhagen et al. (2006) and found to agree within errors. Projected rotational velocities for all cluster members observed with HIRES were found to range from $<9$ to more than $75 \mathrm{~km} \mathrm{~s}^{-1}$. Rotational velocities are not given in Table 1 for the two suspected spectroscopic binaries in the sample (L22 and L9).

\section{ACCRETION DIAGNOSTICS}

In the magnetospheric accretion model, gas from the inner disk edge is channeled along lines of magnetic flux to the stellar surface where its impact upon the photosphere generates hot continuum excess emission with peak temperatures of $\sim 8000$ 11,000 K (Valenti et al. 1993; Hartmann et al. 1998; Muzerolle et al. 1998a). The infalling gas is inferred from inverse $\mathrm{P}$ Cygni line profiles as well as broadened $\mathrm{H} \mathrm{I}, \mathrm{He}$ I, and $\mathrm{Ca}$ II emission lines with velocity widths often exceeding several $100 \mathrm{~km} \mathrm{~s}^{-1}$ (Hamann \& Persson 1992; Batalha \& Basri 1993). Mass accretion rates are most reliably determined using low or intermediate resolution spectrophotometry in the blue (3200$5000 \AA$ ) to measure the hot continuum excess emission directly from the Balmer jump (Valenti et al. 1993; Gullbring et al. 1998; Herczeg \& Hillenbrand 2008), or by using dereddened $U$-band photometry, which correlates strongly with accretion luminosity (Gullbring et al. 1998). The difficulty in using either blue continuum excesses or $U$-band photometry to derive $\dot{M}$ arises from the high optical extinctions often present within star-forming regions and young clusters. Consequently reliable accretion diagnostics in the red have been sought in order to estimate $M$ for stars in heavily extincted regions. In the following sections we examine several accretion diagnostics and apply them to the IC 348 membership sample. Veiling or continuum excess emission is determined near $\lambda 6500$, allowing a direct measurement of accretion luminosity, $L_{\text {acc }}$. We also use extinction-corrected $\mathrm{He}$ I $\lambda 5876, \mathrm{H} \alpha, \mathrm{Ca}$ II $\lambda 8542, \mathrm{~Pa} \beta$, and $\mathrm{Br} \gamma$ line luminosities to estimate $L_{\text {acc }}$ using well-established linear relationships from Muzerolle et al. (1998a, 1998c), Herczeg \& Hillenbrand (in press), and from this investigation. The derived accretion luminosities are then directly proportional to $\dot{M}$ such that

$$
L_{\mathrm{acc}} \sim \frac{G M_{*} \dot{M}}{R_{*}}\left(1-\frac{R_{*}}{R_{\mathrm{in}}}\right)
$$

where the stellar mass and radius estimates are obtained from the evolutionary models of Siess et al. (2000). The factor $\left(1-R_{*} / R_{\text {in }}\right)$ is assigned a value of 0.8 , which assumes an inner disk radius $\left(R_{\mathrm{in}}\right)$ of $5 R_{*}$ (Gullbring et al. 1998).

\subsection{Continuum Excess Emission}

Veiling or continuum excess emission, defined such that $r=F_{\text {exc }} / F_{\text {phot }}$, was determined for the suspected accretors by fitting the depths of several photospheric absorption features with those of a rotationally broadened and artificially veiled standard star of similar spectral type (White \& Hillenbrand 2004). Several $\mathrm{Ca} I$ and $\mathrm{Fe} I$ lines spanning from 6000 and $6500 \AA$ and across multiple HIRES orders were used in the veiling analysis. To determine the continuum excess luminosity, the stellar photospheric flux was derived using the extinctioncorrected, $R$-band mag. If $R$-band photometry was unavailable, the $I$-band mag and intrinsic $V-I$ color were used to determine $M_{R}$ from the pre-main-sequence models of Siess et al. (2000). The photometric zero point of the Cousins $R_{C}$ filter $(2.25 \times$ $10^{-9} \mathrm{erg} \mathrm{s}^{-1} \mathrm{~cm}^{-2} \AA^{-1}$ ) was assumed to derive observed fluxes. To transform the excess fluxes to total accretion luminosity, a multiplicative bolometric correction must be applied to account for accretion-generated flux outside of the $R$-bandpass. We adopt a bolometric correction of 11.1, following the reasoning of White \& Hillenbrand (2004). This correction is the logarithmic average of the two values proposed by Gullbring et al. (1998), 3.5, and of Hartigan \& Kenyon (2003), 35. Once the bolometric correction is applied, the mass accretion rates follow using the stellar mass and radius estimates of the Siess et al. (2000) premain-sequence models. In general, the veiling estimates for the suspected accretors in IC 348 range from near zero up to 0.38 , suggesting that most are lightly veiled when compared with the Class I and Class II sources of Taurus (Gullbring et al. 1998; White \& Hillenbrand 2004). The resulting $\log \dot{M}$ values range from -7.1 to $-8.7 M_{\odot} \mathrm{yr}^{-1}$ and are consistent with other $M$ determinations from the emission line luminosity analysis. Table 3 lists the determined $r$ values as well as the estimated mass accretion rates for the suspected accretors in the IC 348 membership sample.

\section{2. $H \alpha$ Emission}

By tradition, the distinction between CTTSs and weak-line $\mathrm{T}$ Tauri stars (WTTSs) is based upon the strength of $\mathrm{H} \alpha$ emission with the demarcating $W(\mathrm{H} \alpha)$ separating these two classes of pre-main-sequence stars established at $10 \AA$, which roughly corresponds to the detection threshold of early objective prism surveys. While no physical interpretation for this value was originally intended, clear differences in the processes responsible for $\mathrm{H} \alpha$ emission in CTTSs (magnetospheric accretion) and WTTSs (enhanced chromospheric activity) have since been incorporated into their characterizations. Additionally, it is apparent that no single equivalent width can be used to distinguish chromospherically-generated $\mathrm{H} \alpha$ emission from that produced in infalling accretion columns. This is perhaps best demonstrated by the depressed photospheric continuum on either side of $\mathrm{H} \alpha$ among late-type (>K5) stars caused by strong molecular absorption (e.g. TiO) bands. This contrast effect (Basri \& Marcy 1995) causes $\mathrm{H} \alpha$ emission from hot chromospheres to stand out prominently when compared to similar emission among stars of earlier spectral type. For this reason, Martín (1998) suggests that the formal distinction between CTTSs and WTTSs be spectral-type dependent, setting the minimum $W(\mathrm{H} \alpha)$ indicative of accretion at $5 \AA$ for K-type TTSs, $10 \AA$ for M0M2 types, and $20 \AA$ for later M-type stars. Using observations of non-veiled stars, White \& Basri (2003) modify these criteria somewhat, proposing instead that $W(\mathrm{H} \alpha) \geqslant 3 \AA$ for K0-K5 type stars, $\geqslant 10 \AA$ for K7-M2.5 type stars, $\geqslant 20 \AA$ for M3-M5.5 stars, and $\geqslant 40 \AA$ for M6-M7.5 stars to be indicative of accretion processes. White \& Basri (2003) also find that the velocity width of $\mathrm{H} \alpha$ at $10 \%$ peak flux can be effectively used to distinguish between optically veiled and non-veiled stars, i.e. between suspected accretors and nonaccretors. They adopt a $10 \%$ velocity width of $270 \mathrm{~km} \mathrm{~s}^{-1}$ for $\mathrm{H} \alpha$ and conclude that this criterion is a more accurate diagnostic of accretion than either veiling or $\mathrm{W}(\mathrm{H} \alpha)$ given its independence from spectral type and from uncertainties 
Table 3

Stellar Properties and Mass Accretion Rates for IC 348 Members

\begin{tabular}{|c|c|c|c|c|c|c|c|c|c|c|}
\hline Identifier $^{\mathrm{a}}$ & $\begin{array}{c}\text { Mass }^{\mathrm{b}} \\
M_{\odot}\end{array}$ & $\begin{array}{c}\text { Radius }^{\mathrm{b}} \\
R_{\odot}\end{array}$ & $\begin{array}{l}\text { Veiling }^{c} \\
(\lambda 6500)\end{array}$ & $\begin{array}{c}\log \dot{M}^{\mathrm{d}} \\
\text { veiling } \\
M_{\odot} \mathrm{yr}^{-1}\end{array}$ & $\begin{array}{c}\log \dot{M}^{\mathrm{e}} \\
\text { He I } \lambda 5876 \\
M_{\odot} \mathrm{yr}^{-1}\end{array}$ & $\begin{array}{c}\log \dot{M}^{\mathrm{f}} \\
\mathrm{H} \alpha \\
M_{\odot} \mathrm{yr}^{-1}\end{array}$ & $\begin{array}{c}\log \dot{M}^{\mathrm{g}} \\
\mathrm{Ca} \text { II } \lambda 8542 \\
M_{\odot} \mathrm{yr}^{-1}\end{array}$ & $\begin{array}{c}\log \dot{M}^{\mathrm{h}} \\
\mathrm{Pa} \beta \\
M_{\odot} \mathrm{yr}^{-1}\end{array}$ & $\begin{array}{c}\log \dot{M}^{\mathrm{i}} \\
\operatorname{Br} \gamma \\
M_{\odot} \mathrm{yr}^{-1}\end{array}$ & $\begin{array}{c}\log \dot{M}^{\mathrm{j}} \\
\text { Mean } \\
M_{\odot} \mathrm{yr}^{-1}\end{array}$ \\
\hline L8012B & 2.25 & 1.57 & . & $\ldots$ & $\ldots$ & -7.45 & $\ldots$ & -8.08 & -7.42 & $-7.65 \pm 0.37$ \\
\hline L5 & 2.08 & 2.82 & 0.18 & -7.10 & -7.61 & -7.20 & -7.55 & -7.04 & -6.86 & $-7.23 \pm 0.30$ \\
\hline L31 & 1.89 & 2.79 & 0.02 & -7.56 & $\ldots$ & -6.56 & -8.14 & -7.11 & -7.20 & $-7.31 \pm 0.58$ \\
\hline L21 & 1.68 & 2.02 & 0.05 & -7.96 & -8.54 & -8.63 & -8.46 & -8.31 & -8.15 & $-8.34 \pm 0.25$ \\
\hline L40 & 0.84 & 1.60 & 0.38 & -7.57 & -7.70 & -7.30 & -7.62 & -7.52 & -7.61 & $-7.54 \pm 0.14$ \\
\hline L37 & 0.82 & 1.86 & 0.14 & -7.86 & -8.54 & -8.67 & -7.92 & -8.30 & -8.16 & $-8.24 \pm 0.32$ \\
\hline L36 & 0.81 & 2.09 & 0.20 & -7.52 & -8.61 & -8.30 & -7.94 & $<-9.21$ & $<-9.67$ & $-8.10 \pm 0.47$ \\
\hline L41 & 0.77 & 1.14 & 0.21 & -8.51 & -8.70 & -8.51 & -8.46 & -8.74 & -8.68 & $-8.60 \pm 0.12$ \\
\hline L32 & 0.70 & 1.71 & 0.37 & -7.55 & -7.71 & -7.65 & -8.10 & -8.09 & -8.21 & $-7.89 \pm 0.27$ \\
\hline L26 & 0.71 & 1.58 & 0.25 & -7.82 & -7.30 & -7.48 & -7.85 & -7.66 & -8.07 & $-7.70 \pm 0.28$ \\
\hline L61 & 0.62 & 1.44 & 0.27 & -7.71 & -8.60 & -8.21 & -8.02 & $<-9.14$ & -9.05 & $-8.31 \pm 0.52$ \\
\hline L9024 & 0.55 & 1.62 & 0.11 & -8.20 & -8.40 & -8.66 & -8.33 & -8.62 & -8.90 & $-8.51 \pm 0.26$ \\
\hline L9060 & 0.54 & 0.95 & $\ldots$ & $\ldots$ & $\ldots$ & $\ldots$ & $\ldots$ & $<-10.14$ & $<-9.90$ & $\ldots$ \\
\hline L67 & 0.45 & 1.10 & 0.09 & -8.73 & -8.74 & -8.68 & -8.72 & $\ldots$ & $\ldots$ & $-8.72 \pm 0.03$ \\
\hline
\end{tabular}

Notes.

a Identifier from Luhman et al. (2003) cluster census.

b Stellar mass and radius estimates obtained from the pre-main-sequence models of Siess et al. (2000).

${ }^{\mathrm{c}}$ Veiling at $\lambda 6500$, defined such that $r=F_{\text {exc }} / F_{\text {phot }}$.

${ }^{\mathrm{d}} \dot{M}$ from $R$-band veiling analysis.

e $\dot{M}$ from He I $\lambda 5876$ analysis.

${ }^{\mathrm{f}} \dot{M}$ from $\mathrm{H} \alpha$ analysis.

g $\dot{M}$ from HIRES Ca II $\lambda 8542$ analysis.

h $\dot{M}$ from $\mathrm{Pa} \beta$ analysis.

i $\dot{M}$ from Br $\gamma$ analysis.

j Adopted mean $\log \dot{M}$ and $1 \sigma$ uncertainty.

introduced when adopting photospheric template spectra for veiling analysis.

The White \& Basri (2003) H $\alpha$ velocity width criterion was applied to the 33 members of IC 348 observed with HIRES, identifying 10 suspected accretors ( 9 primordial disk candidates and 1 weak or transition disk candidate) within the sample. Two additional primordial disk candidates (L37 and L32) and one weak or transition disk object (L67) were found with $10 \%$ velocity widths of 256,265 , and $248 \mathrm{~km} \mathrm{~s}^{-1}$, respectively, but are almost certainly accreting given the presence of strong [O I] $\lambda 6300$ and [S II] $\lambda 6731$ emission, indicators of outflow activity and stellar or disk winds. The SpeX observations of L37 reveal strong Ca II $\lambda \lambda 8498,8542$, and 8662 emission (equivalent widths of $-4.10,-3.37,-2.95 \AA$, respectively) compared to marginal emission during the later epoch HIRES observations $(-0.39,-0.55$, and $-0.32 \AA)$. Such differences demonstrate the temporal variability of emission line strengths, possibly stemming from stochastic accretion processes. We suggest that the velocity width criterion of White \& Basri (2003) be reduced to $\sim 250 \mathrm{~km} \mathrm{~s}^{-1}$ to include these disk-bearing stars. Shown in Figure 9 are the $\mathrm{H} \alpha$ emission profiles for the 13 suspected accretors in the IC 348 sample. The wings of the line profiles extend beyond $400 \mathrm{~km} \mathrm{~s}^{-1}$ in the two strongest accretors, L40 and L5, but in general are limited to $\sim 300 \mathrm{~km} \mathrm{~s}^{-1}$ or less. The $\mathrm{H} \alpha$ line profiles exhibit significantly diverse morphologies that presumably arise from differing $\dot{M}$ values, gas temperatures, and geometries (inclinations and magnetospheric radii). Radiative transfer models of $\mathrm{H} \alpha$ emission profiles by Muzerolle et al. (1998b) are capable of reproducing some of the observed morphologies, but fail to produce the high velocity wings observed in strongly accreting stars. Rather than tracing high velocity gas, Muzerolle et al. (1998b) suggest that the line wings are generated by the linear Stark effect, which becomes a dominant line broadening mechanism at high temperatures and ion densities. Later radiative transfer models by Muzerolle et al. (2001) and Kurosawa et al. (2006) successfully reproduced the high velocity wings of $\mathrm{H} \alpha$ by assuming a Voigt line profile and a damping constant $(\Gamma)$ that includes natural broadening, van der Waals broadening, and the Stark effect.

Although the velocity width of $\mathrm{H} \alpha$ is a well-established accretion diagnostic, the use of $\mathrm{H} \alpha$ line flux as a correlate of accretion luminosity is complicated by a number of factors that influence line emission including magnetospheric structure, temperature, and density (Muzerolle et al. 1998b). At high mass accretion rates, $\mathrm{H} \alpha$ emission is expected to be optically thick and therefore insensitive to gas density. Observed emission from accreting stars is also not necessarily dominated by the accretion flow and can be significantly impacted by system geometry. Given these caveats, we examine the $\mathrm{H} \alpha$ emission line fluxes for 14 members of Taurus-Auriga that have well-established $L_{\text {acc }}$ and $\dot{M}$ values from the blue continuum excess surveys of Valenti et al. (1993) and Gullbring et al. (1998). Of the 14 Taurus-Auriga members examined, 10 (AA Tau, BP Tau, DG Tau, DL Tau, DN Tau, DR Tau, DS Tau, GK Tau, RW Aur, UY Aur) have $\mathrm{H} \alpha$ equivalent widths from Muzerolle et al. (1998a) and 4 (CY Tau, DK Tau, DO Tau, GI Tau) were observed with HIRES as part of this investigation. All are K3M0 type stars with $\log \dot{M}$ ranging from -5.1 to $-8.5 M_{\odot} \mathrm{yr}^{-1}$. To determine $\mathrm{H} \alpha$ luminosities for these stars, we use the measured equivalent widths, the extinction-corrected $R$-band magnitudes, the spectral type dependent flux ratio between $\mathrm{H} \alpha$ and the effective wavelength of the $R$-band filter (from the spectrophotometry of O'Connell 1973), and an assumed distance of 140 pc for Taurus-Auriga (Kenyon et al. 1994). Figure 10(a) plots accretion luminosity as a function of $\mathrm{H} \alpha$ emission line flux. Although significant scatter is apparent, the fit 

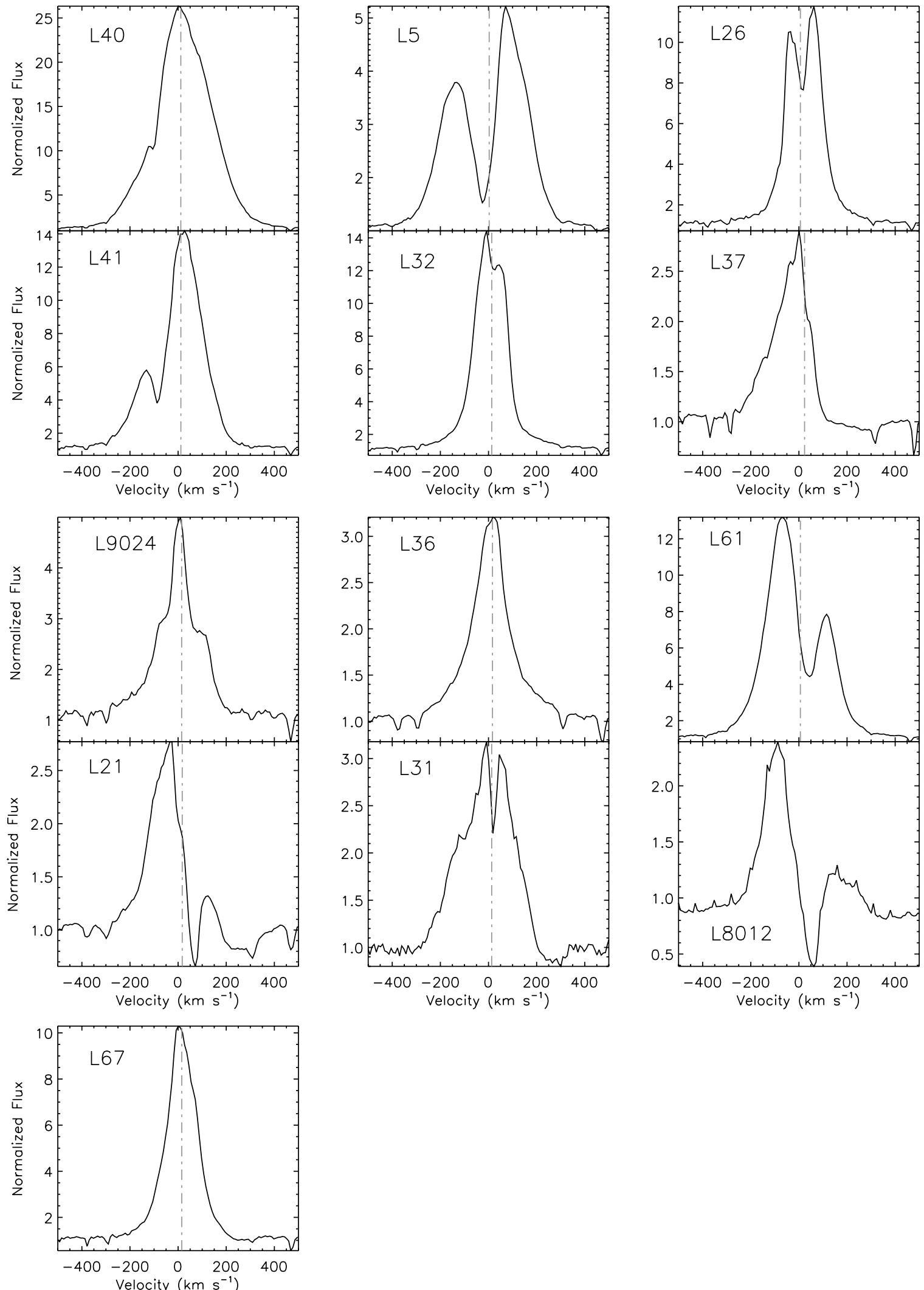

Figure 9. Observed $\mathrm{H} \alpha$ emission profiles for the 13 suspected accretors in the IC 348 membership sample. Accretion determinations were made using the $10 \%$ velocity width criterion of White \& Basri (2003), which is independent of veiling and assumed spectral type. All of the suspected accretors exhibit other indicators of accretion activity, including [O I] $\lambda 6300$ and [S II] $\lambda 6731$ emission, as well as permitted transitions of He I and Ca II. The vertical dot-dashed line indicates the measured radial velocity for each star.

spans nearly three orders of magnitude in accretion luminosity, despite the non-simultaneous nature of the $\mathrm{H} \alpha$ observations and the blue continuum excess surveys. The resulting linear regression fit between the $\mathrm{H} \alpha$ luminosities and $L_{\text {acc }}$ is given by 

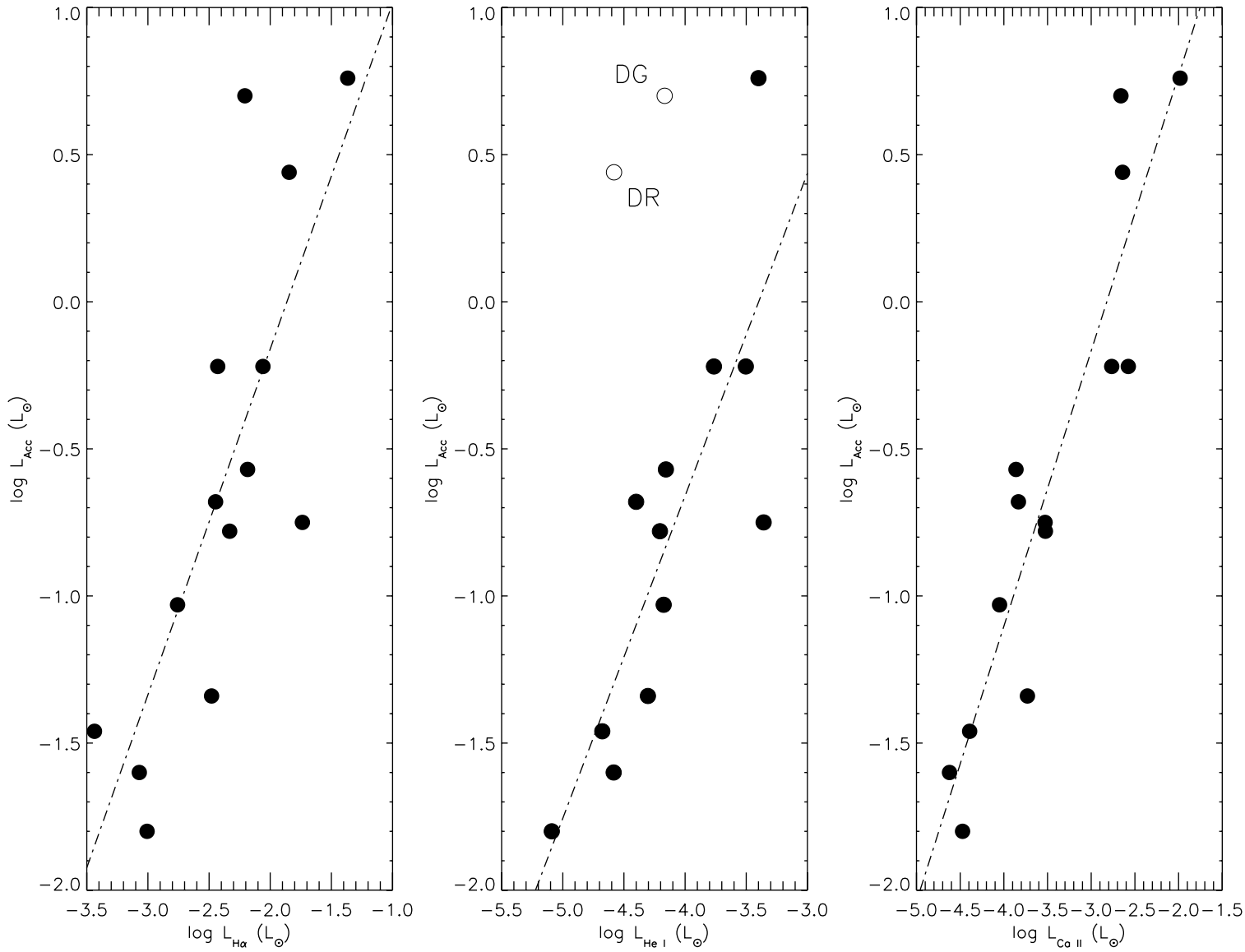

Figure 10. Accretion luminosity $\left(L_{\mathrm{acc}}\right)$ plotted as a function of $\mathrm{H} \alpha$ (left), $\mathrm{He} \mathrm{I} \lambda 5876$ (center), and $\mathrm{Ca}$ II $\lambda .8542$ (right) emission line luminosities for 14 members of Taurus-Auriga having well-established $L_{\text {acc }}$ and $\dot{M}$ determinations from the blue continuum excess surveys of Valenti et al. (1993) and Gullbring et al. (1998). The derived linear regression fits given here are superposed. DG Tau and DR Tau were removed from the analysis for He I $\lambda 5876$ given their high mass accretions rates ( $\log \dot{M} \sim-5.7$ and $-5.1 M_{\odot} \mathrm{yr}^{-1}$, respectively) and moderate He I $\lambda 5876$ emission strengths.

$\log \left(L_{\mathrm{acc}} / L_{\odot}\right)=(1.18 \pm 0.26) \log \left(L_{H \alpha} / L_{\odot}\right)+(2.19 \pm 0.64)$

Herczeg \& Hillenbrand (in press) derive a similar relationship between $L_{H \alpha}$ and $L_{\text {acc }}$ for several low-mass stars and brown dwarfs in Taurus-Auriga by simultaneously measuring blue continuum excesses using the Balmer discontinuity and $\mathrm{H} \alpha$ emission line fluxes. Their sources all have $\operatorname{low} \log \dot{M}$ values, ranging from -9 to $-11 M_{\odot} \mathrm{yr}^{-1}$, effectively complementing the higher mass accretion rates predicted for the more massive Taurus members. Their linear relationship between $L_{\text {acc }}$ and $L_{H \alpha}$ is given by

$$
\log \left(L_{\text {acc }} / L_{\odot}\right)=(1.31 \pm 0.16) \log \left(L_{H \alpha} / L_{\odot}\right)+(2.27 \pm 0.70) .
$$

The derived linear coefficients for the stellar and substellar fits are within uncertainties. We adopt the relationship for the more massive Taurus-Auriga members to estimate $L_{\text {acc }}$ for the IC 348 members. The predicted $L_{\text {acc }}$ values are then used to estimate $\dot{M}$ using stellar mass and radius estimates from the models of Siess et al. (2000). The resulting $\log \dot{M}$ values range from -6.56 to $-8.68 M_{\odot} \mathrm{yr}^{-1}$ and are listed in Table 3. The highest mass accretion rate is that of the deeply embedded star, L31 $\left(A_{V} \sim\right.$ $12 \mathrm{mag})$. Uncertainty in the $R$-band extinction correction applied to this star may induce an artificially high $\mathrm{H} \alpha$ luminosity and consequently $\log \dot{M}$, as suggested by the somewhat lower $\log \dot{M}$ values determined from the $\mathrm{Pa} \beta$ and $\mathrm{Br} \gamma$ analysis (Section 4.3).
The magnetospheric accretion models of Muzerolle et al. (1998b) and Kurosawa et al. (2006) assume a $0.5 M_{\odot}$ star with a radius of $2.0 R_{\odot}$ and peak magnetospheric temperatures ranging from 6500 to $10,000 \mathrm{~K}$. Shown in Figure 11 are the $\mathrm{H} \alpha$ emission line fluxes for 9 suspected accretors in IC 348 that have masses and radii ranging from $0.45-0.8 M_{\odot}$ and from 1.1$2.1 R_{\odot}$, respectively, plotted as a function of $\log \dot{M}$. Although not exact analogs to the stellar model assumed by Muzerolle et al. (1998b) and Kurosawa et al. (2006), these stars are similar in mass and radius. Superposed in Figure 11 are the magnetospheric accretion model $\mathrm{H} \alpha$ fluxes of Muzerolle et al. (1998b), which vary the inner and outer magnetospheric radii from 2.23.0 and 4.0-6.0 $R_{*}$, respectively. Also superposed are the models of Kurosawa et al. (2006), transformed from the given equivalent widths to $\mathrm{H} \alpha$ emission line fluxes using the models of Siess et al. (2000) for a 1.1 Myr old, $0.5 M_{\odot}$ star (the radius of which is $\sim 2.0 R_{\odot}$ ) to estimate $M_{R}$ and subsequently, $L_{H \alpha}$. Muzerolle et al. (2001) suggest that by comparing their models with observed line fluxes and accretion rates, it is possible to place constraints upon gas temperatures. The suspected accretors in IC 348 cluster around the highest temperature magnetospheric models, $T_{\mathrm{mag}}=10,000 \mathrm{~K}$. Incorporating other $\mathrm{H}$ I emission lines (e.g., Balmer, Paschen, Brackett series) and ion species (e.g. He I, Ca II, Na I) into future radiative transfer models of magnetospheric accretion should provide better constraints for the physical conditions present within accreting systems (Muzerolle et al. 1998b). 


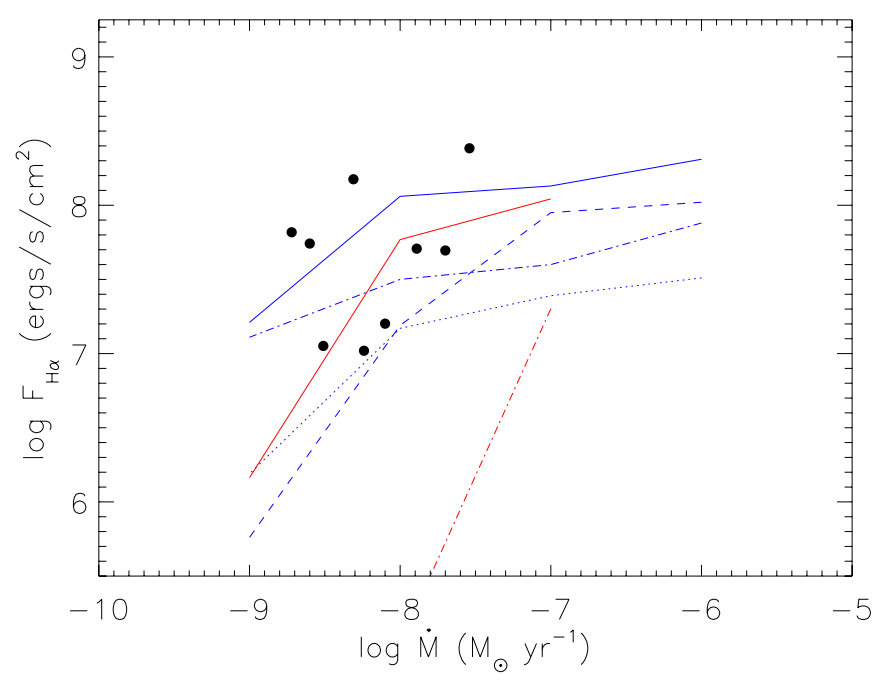

Figure 11. Extinction-corrected $\mathrm{H} \alpha$ emission line fluxes for nine suspected accretors in the IC 348 sample plotted against mass-accretion rates derived here. Superposed are the magnetospheric accretion models of Muzerolle et al. (1998b) in blue and of Kurosawa et al. (2006) in red, derived assuming a $0.5 M_{\odot}, 2.0 R_{\odot}$ star. The IC 348 members shown all have similar masses and radii $\left(0.45-0.8 M_{\odot}, 1.1-2.1 R_{\odot}\right)$. The solid blue curve represents the Muzerolle et al. (1998b) model having inner $\left(R_{\mathrm{mi}}\right)$ and outer $\left(R_{\mathrm{mo}}\right)$ magnetospheric radii of 4.0 and $6.0 R_{*}$, respectively, and a magnetospheric temperature $\left(T_{\mathrm{mag}}\right)$ of $10,000 \mathrm{~K}$. The blue dot-dash line is the Muzerolle et al. (1998b) model corresponding to $R_{\mathrm{mi}}=2.2, R_{\mathrm{mo}}=3.0 R_{*}$, and $T_{\mathrm{mag}}=10,000 \mathrm{~K}$. The blue dashed and dotted lines represent the Muzerolle et al. (1998b) models having identical radii as the models described above, but with $T_{\mathrm{mag}}=8000 \mathrm{~K}$. The red solid line is the Kurosawa et al. (2006) magnetospheric accretion model for $T_{\mathrm{mag}}=10,000 \mathrm{~K}$ and the red dot-dashed line is that for $T_{\mathrm{mag}}=6500 \mathrm{~K}$.

(A color version of this figure is available in the online journal)

\section{3. $\mathrm{Pa} \beta$ and $\mathrm{Br} \gamma$ Emission}

$\operatorname{Pa} \beta(n=5-3)$ and $\operatorname{Br} \gamma(n=7-4)$ provide higher upper level H I transitions that may better probe $\dot{M}$ than the optically thick Balmer lines. These near-infrared transitions are also significantly less impacted by extinction than Balmer line emission, a critical factor for the many embedded sources in IC 348. Muzerolle et al. (1998c) observe $\mathrm{Pa} \beta$ and $\mathrm{Br} \gamma$ emission in a sample of CTTSs in the Taurus-Auriga star-forming region and find their line luminosities to be well correlated with $L_{\text {acc }}$. In their survey of 30 CTTSs with established $\dot{M}$ values derived either from the blue continuum excess spectrophotometry of Gullbring et al. (1998) and Calvet \& Gullbring (1998) or the $U$-band photometry of Hartmann et al. (1998), Muzerolle et al. (1998c) find that all but one CTTS exhibit $\mathrm{Pa} \beta$ emission and 26 of 30 show $\mathrm{Br} \gamma$ emission. A general trend of increasing $\mathrm{Pa} \beta$ and $\mathrm{Br} \gamma$ equivalent width with $\dot{M}$ was also noted by Muzerolle et al. (1998c), although significant scatter is evident between these parameters. A more physically meaningful indicator of emission strength is emission line flux estimated for $\mathrm{Pa} \beta$ and $\mathrm{Br} \gamma$ using the extinction corrected $J$ - and $K$-band photometry from Kenyon \& Hartmann (1995), respectively. The resulting line fluxes for both were found to be well correlated with log $\dot{M}$ (see their Figure 3), particularly when considering just stars with spectrophotometric mass accretion rates. Muzerolle et al. (1998c) also find that $L_{\mathrm{acc}}$, which is independent of estimates for stellar mass and radius, correlates strongly with $\mathrm{Pa} \beta$ and $\mathrm{Br} \gamma$ line luminosities $\left(L_{\mathrm{Pa} \beta}\right.$ and $\left.L_{\mathrm{Br} \gamma}\right)$. The resulting least-squares fits are given by

$\log \left(L_{\mathrm{acc}} / L_{\odot}\right)=(1.14 \pm 0.16) \log \left(L_{P a \beta} / L_{\odot}\right)+(3.15 \pm 0.58)$ and

$$
\log \left(L_{\mathrm{acc}} / L_{\odot}\right)=(1.26 \pm 0.19) \log \left(L_{\mathrm{Br} \gamma} / L_{\odot}\right)+(4.43 \pm 0.79) .
$$

To ensure that the line luminosities are not simply scaling with photospheric emission, Muzerolle et al. (1998c) examine the relationship between $J$ - and $K$-band mag and $\log \left(L_{\text {acc }}\right)$, as well as between extinction $\left(A_{V}\right)$ and $\log \left(L_{\text {acc }}\right)$. Only the $K$ band mag exhibited a detectable trend, which very likely arises from inner disk dust emission.

For the 39 members of IC 348 observed with SpeX, nonexcess stars of similar spectral type (several from the IRTF spectral atlas) were selected as templates in order to subtract $\mathrm{Pa} \beta$ and $\mathrm{Br} \gamma$ photospheric absorption. In total 11 of 39 cluster members examined were found with $\mathrm{Pa} \beta$ and/or $\mathrm{Br} \gamma$ emission, all of which are suspected accretors based upon the $\mathrm{H} \alpha$ velocity width analysis. Only one accretor and primordial disk candidate does not exhibit either $\mathrm{Pa} \beta$ or $\mathrm{Br} \gamma$ emission, the $\mathrm{K} 6$ type star L36. This could result from the non-simultaneity of the optical and near-infrared observations and the stochastic nature of accretion processes, the use of an incorrect template star to remove stellar photospheric absorption, or perhaps the lack of high-resolution, near-infrared spectroscopy, which may have revealed unresolved emission. L36 does exhibit weak He I $\lambda 10830$ emission, but is almost certainly accreting given the presence of strong $\mathrm{H} \alpha$, [O I] $\lambda 6300$, and [S II] $\lambda 6731$ emission. One other primordial disk candidate which was not observed with HIRES was found to lack $\mathrm{Pa} \beta$ and $\mathrm{Br} \gamma$ emission, the faint $(V \sim 18.29)$ M0-type star L9060. The SpeX SXD spectrum is weak and only the three reddest orders could be extracted. While strong $\mathrm{Pa} \beta$ or $\mathrm{Br} \gamma$ emission from this star can be effectively ruled out, weak emission may be present, but simply below the detection threshold in the low signal-to-noise spectrum. Herbig (1998) noted strong $\mathrm{H} \alpha$ emission from this star (identified as IfA $\mathrm{H} \alpha 24)$, with $\mathrm{W}(\mathrm{H} \alpha)=51 \AA$ a definitive accretor for its M0 spectral type.

Shown in Figure 12 are the emission line profiles of $\mathrm{Pa} \gamma$, $\mathrm{Pa} \beta$, and $\mathrm{Br} \gamma$ for 12 suspected accretors in the IC 348 sample. $\mathrm{Pa} \beta$ and $\mathrm{Br} \gamma$ line luminosities for these stars were determined directly from the flux calibrated spectra, which are expected to have flux uncertainties of $\sim 15-20 \%$. These values were compared with line luminosity estimates determined using the measured equivalent widths for these features and the $J$ - and $K_{S}$-band magnitudes from the 2MASS point source catalog, corrected for extinction. Both line luminosity estimates for $\mathrm{Pa} \beta$ and $\mathrm{Br} \gamma$ were found to be in agreement for all stars. We list in Table 2 those values determined from the flux-calibrated spectra. The resulting line luminosities were then transformed into $L_{\text {acc }}$ using the linear relationships of Muzerolle et al. (1998c) and into $\dot{M}$ using the individual stellar masses and radii from the models of Siess et al. (2000). Presented in Table 3 are the $\log \dot{M}$ values determined from $L_{\mathrm{Pa} \beta}$ and $L_{\mathrm{Br} \gamma}$ for the suspected IC 348 accretors. Derived accretion rates are found to be in agreement with each other and with those derived from the veiling (Section 4.1) H $\alpha$ (Section 4.2), He I $\lambda 5876$ (Section 4.4), and $\mathrm{Ca}$ II $\lambda 8542$ (Section 4.5) analyses.

In their radiative transfer analysis of the magnetospheric infall zone of accreting TTSs, Muzerolle et al. (1998b) model line profiles for both $\mathrm{Pa} \beta$ and $\mathrm{Br} \gamma$ emission. Even for these transitions, the upper levels of which are presumably populated in closer proximity to the star than the $n=3$ level responsible for $\mathrm{H} \alpha$ emission, $\mathrm{Pa} \beta$ and $\mathrm{Br} \gamma$ remain optically thick except 

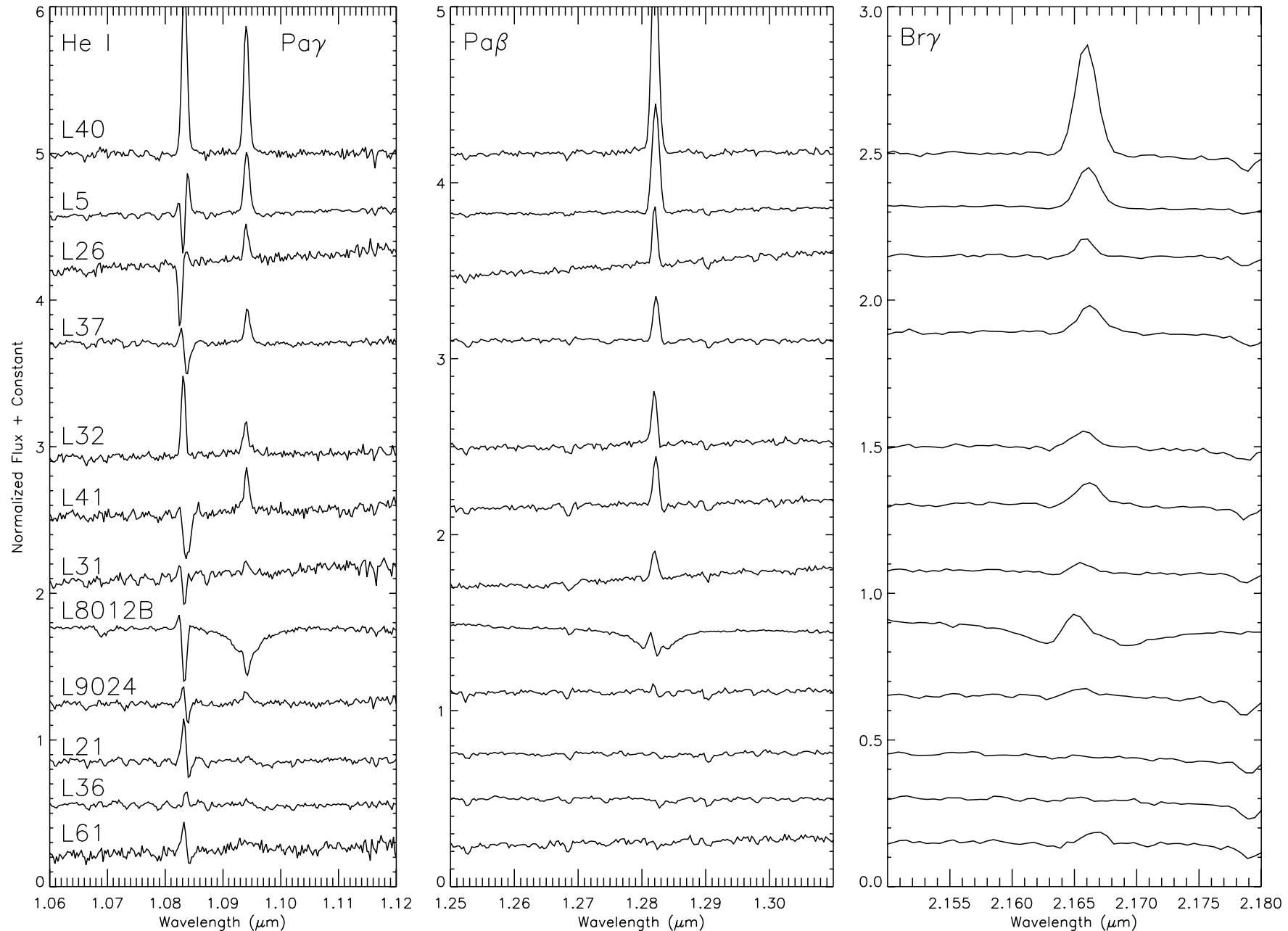

Figure 12. Near-infrared spectra of the 12 suspected accretors in the IC 348 membership sample obtained using SpeX centered upon Pa $\gamma$, Pa $\beta$, and $\mathrm{Br} \gamma$. Profiles shown here have been normalized using featureless regions of the continuum adjacent to each emission line. With the exceptions of L36 and L9060 (not shown), all primordial disk candidates and suspected accretors exhibit $\mathrm{Pa} \beta$ and/or $\mathrm{Br} \gamma$ emission after correcting for photospheric absorption by subtracting the spectrum of a non-excess template star of similar spectral type. Also apparent near Pa $\gamma$ is the He I $\lambda 10830$ transition, which exhibits significant structure even at these low dispersions.

at the lowest mass accretion rates and temperatures (Muzerolle et al. 1998b). Shown in Figures 13 and 14 are the observed line fluxes for $\mathrm{Pa} \beta$ and $\mathrm{Br} \gamma$, respectively, plotted as a function of $\log \dot{M}$ for the 7 suspected accretors in IC 348 having detected near-infrared line emission as well as masses and radii similar to those of the stellar model assumed by Muzerolle et al. (1998b) $\left(0.5 M_{\odot}, 2.0 R_{\odot}\right)$. Superposed are the $\mathrm{Pa} \beta$ and $\mathrm{Br} \gamma$ line fluxes and mass accretion rates from the magnetospheric accretion models of Muzerolle et al. (1998b). These models appear to be in somewhat better agreement with observed line fluxes than the $\mathrm{H} \alpha$ models from Section 4.2, possibly due to the significantly reduced extinction effects in the near-infrared. Muzerolle et al. (1998b) attempt to constrain the temperature distribution of the magnetosphere, and find that if gas temperatures and $\dot{M}$ are too high, the continuum becomes optically thick and effectively veils emission arising from the accretion column. If magnetospheric temperatures are too low, no line emission is produced for $\log \dot{M}<-8 M_{\odot} \mathrm{yr}^{-1}$. The resulting range of allowed gas temperatures precludes $T_{\mathrm{mag}} \geqslant 10,000 \mathrm{~K}$ for $\log \dot{M} \geqslant-8 M_{\odot} \mathrm{yr}^{-1}$. This is not observed, however, among the $\mathrm{Pa} \beta$ line fluxes which cluster around the magnetospheric accretion models characterized by gas temperatures of $10,000 \mathrm{~K}$. For the $\mathrm{Br} \gamma$ line fluxes, stars having low $\dot{M}$ do fall upon the higher temperature models, as expected, while stars with $\log \dot{M} \geqslant-8 \mathrm{M}_{\odot} \mathrm{yr}^{-1}$ lie between models having temperatures of $\sim 8000$ and $10,000 \mathrm{~K}$. It is possible that $\mathrm{Br} \gamma$ emission is sufficiently optically thin that observed line fluxes are probing the temperature structure of the magnetosphere. Given the large number of uncertainties involved in the determination of emission line fluxes and $\dot{M}$, the relative agreement between the observed $\mathrm{Pa} \beta$ and $\mathrm{Br} \gamma$ line fluxes and the Muzerolle et al. (1998b) magnetospheric accretion models suggests that these transitions may probe accretion flows better than Balmer line emission.

\subsection{He I Emission}

Despite its abundance, helium does not produce strong photospheric features in stars later than $\sim \mathrm{A}$ spectral type because of its high excitation potential, $>20 \mathrm{eV}$. For this reason in solar mass and less massive main-sequence stars, observed transitions of helium are considered to be chromospheric in nature. Among pre-main-sequence stars, broadened emission line profiles of He I transitions have been linked with magnetospheric accretion flows, as demonstrated by Muzerolle et al. (1998a) in their high-resolution spectroscopic survey of 11 CTTSs in the TaurusAuriga star-forming region. Beristain et al. (2001) suggest that 


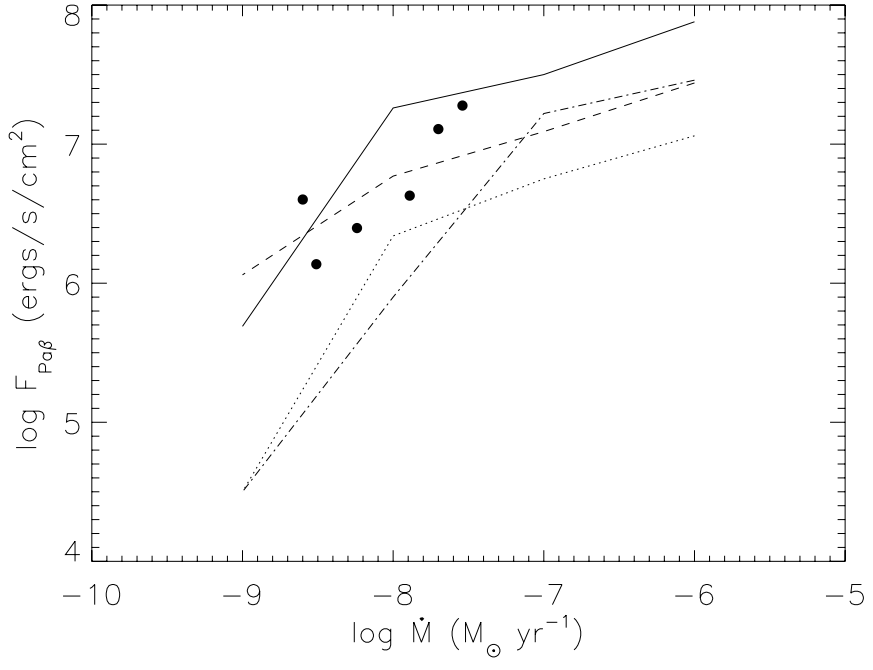

Figure 13. Extinction-corrected $\mathrm{Pa} \beta$ emission line fluxes for six suspected accretors in the IC 348 membership sample with detected emission, plotted against mass accretion rates derived here. Superposed are the magnetospheric accretion models of Muzerolle et al. (1998b) with the solid curve corresponding to $T_{\mathrm{mag}}=10,000 \mathrm{~K}, R_{\mathrm{mi}}=4.0$ and $R_{\mathrm{mo}}=6.0 R_{*}$, the dashed curve to $T_{\mathrm{mag}}=10,000 \mathrm{~K}, R_{\mathrm{mi}}=2.2$ and $R_{\mathrm{mo}}=3.0 R_{*}$, the dot-dashed curve to $T_{\mathrm{mag}}=8000 \mathrm{~K}, R_{\mathrm{mi}}=4.0$ and $R_{\mathrm{mo}}=6.0 R_{*}$, and the dotted curve to $T_{\mathrm{mag}}=8000 \mathrm{~K}, R_{\mathrm{mi}}=2.2$ and $R_{\mathrm{mo}}=3.0 R_{*}$. The Muzerolle et al. (1998b) models assume a $0.5 M_{\odot}, 2.0 R_{\odot}$ accreting star. All IC 348 members shown have similar masses and radii $\left(0.45-0.8 M_{\odot}, 1.1-2.1 R_{\odot}\right)$. The models effectively bracket the observed data, with the higher magnetospheric temperatures providing the best fits.

the narrow component of $\mathrm{He} \mathrm{I} \lambda 5876$ emission is produced by decelerating gas emerging from the accretion shock, while the broad component is composite in nature with possible origins from the accretion column and a disk wind. Weakly accreting stars (e.g., AA Tau, DN Tau, GK Tau), however, do not exhibit the velocity broadened components of He I emission, thereby limiting the effectiveness of profile decomposition alone in identifying low-mass accretion rate stars. The emission line flux of He I $\lambda 5876$, however, was shown by Muzerolle et al. (1998a) to be moderately well correlated with mass accretion rate. Herczeg \& Hillenbrand (in press) find a much stronger correlation between $\mathrm{He}$ I $\lambda 5876$ flux and $L_{\mathrm{acc}}$ in their spectrophotometric survey of low-mass Taurus-Auriga members.

Three HeI transitions were examined in this survey as potential accretion diagnostics: He I $\lambda \lambda 5876,6678$ observed at high resolution with HIRES and He I $\lambda 10830$ observed at moderate resolution with SpeX. He I $\lambda 5876$ is found in emission for all suspected accretors in the IC 348 sample with the exceptions of L31 (weak emission possible), which has a low signal-to-noise spectrum, and the early-type star L8012B. He I $\lambda 5876$ line profiles for these stars are shown in Figure 15. In contrast with $\mathrm{He}$ I $\lambda 5876, \lambda 6678$ is found predominantly with blue-shifted absorption profiles in stars L5 (weak emission component present), L26, L37 (weak emission component present), L36, L21, L31, and L8012B. The blueshifted absorption is possibly associated with a disk or accretion driven wind. The He I $\lambda 6678$ profiles for the remaining accretors are found with narrow emission profiles. These findings are in conflict with Muzerolle et al. (1998a) who state that emission line profiles of $\mathrm{He}$ I $\lambda 5876, \lambda 6678$, and $\lambda 7065$ for their sample of 11 CTTSs in Taurus were virtually identical, with $\lambda 5876$ being the dominant He I spectral feature. For the echelle and crossdisperser angles selected for the IC 348 survey, He I $\lambda 7065$ lies just off the edge of the red chip of HIRES, precluding

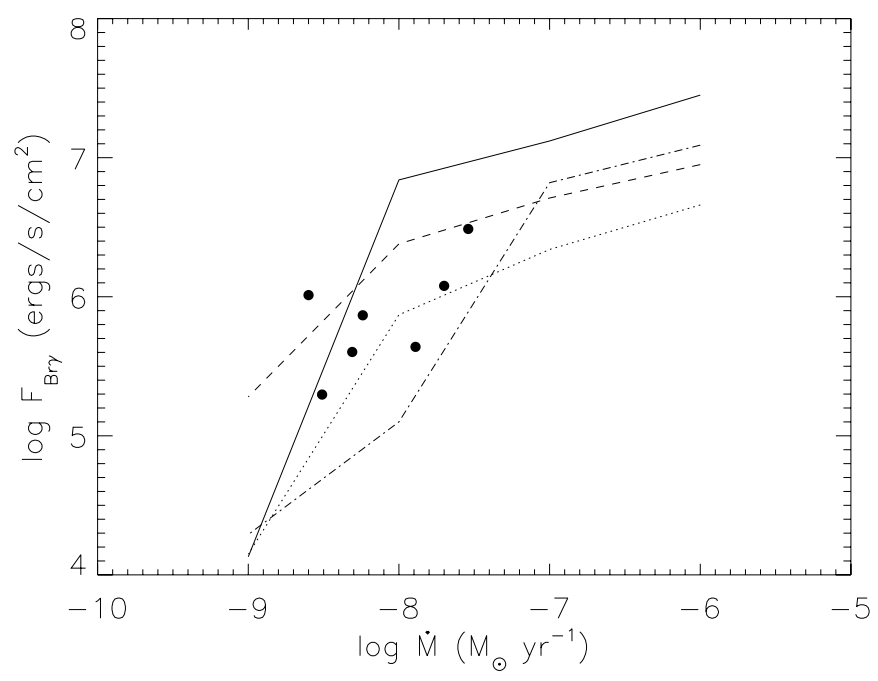

Figure 14. Extinction-corrected $\mathrm{Br} \gamma$ emission line fluxes for seven suspected accretors in the IC 348 membership sample plotted against mass accretion rates derived here. Superposed are the $\mathrm{Br} \gamma$ emission line fluxes magnetospheric accretion models of Muzerolle et al. (1998b) plotted as in Figure 13. IC 348 members having lower mass accretion rates are better constrained by the higher magnetospheric temperature models of Muzerolle et al. (1998b), while observed stars having larger $\dot{M}$ values fall along the lower temperature models. It is possible that $\mathrm{Br} \gamma$ emission is sufficiently optically thin that observed line fluxes are sensitive to gas density.

analysis here. The He I $\lambda 5876$ profiles for the strongest accretors in the IC 348 sample (L5, L40) possess velocity widths in excess of $200 \mathrm{~km} \mathrm{~s}^{-1}$, but most appear narrow with FWHM $100 \mathrm{~km} \mathrm{~s}^{-1}$ or less.

To determine accretion luminosities using measured He I $\lambda 5876$ line fluxes, we follow a similar procedure to that adopted for $\mathrm{H} \alpha$ emission in Section 4.2. A linear relationship between He I $\lambda 5876$ emission line luminosity $\left(L_{\lambda 5876}\right)$ and $L_{\text {acc }}$ is derived using 12 members of Taurus-Auriga that have well-established $L_{\text {acc }}$ values from blue continuum excess measurements. To determine $L_{\lambda 5876}$, we use the measured equivalent widths (from Muzerolle et al. 1998a or this investigation), the extinctioncorrected $V$-band magnitudes, the spectral type dependent flux ratio between $\lambda 5876$ and the effective wavelength of the $V$ band filter, and an assumed distance of 140 pc for TaurusAuriga. Figure 10(b) plots $L_{\text {acc }}$ as a function of $L_{\lambda 5876}$ for the 12 Taurus-Auriga members. Again some scatter is apparent, but the linear fit spans nearly three orders of magnitude in accretion luminosity, despite the non-simultaneous nature of the He I $\lambda 5876$ and the blue continuum excess observations. The resulting linear regression fit is given by

$\log \left(L_{\mathrm{acc}} / L_{\odot}\right)=(1.10 \pm 0.23) \log \left(L_{\lambda 5876} / L_{\odot}\right)+(3.73 \pm 0.98)$.

Two stars were removed from the $L_{\lambda 5876}-L_{\text {acc }}$ analysis, DG and DR Tau, both of which have high mass accretions rates (log $\dot{M} \sim-5.7$ and $-5.1 M_{\odot} \mathrm{yr}^{-1}$, respectively) and moderate He I $\lambda 5876$ emission strengths. For the low-mass stellar and brown dwarf members of Taurus-Auriga, Herczeg \& Hillenbrand (in press) derive a similar relationship between $L_{\lambda 5876}$ and $L_{\text {acc }}$ such that

$\log \left(L_{\mathrm{acc}} / L_{\odot}\right)=(1.46 \pm 0.12) \log \left(L_{\lambda 5876} / L_{\odot}\right)+(5.30 \pm 0.70)$.

The coefficients for the stellar and substellar linear fits are again within uncertainties. We adopt the former to derive $L_{\text {acc }}$ 

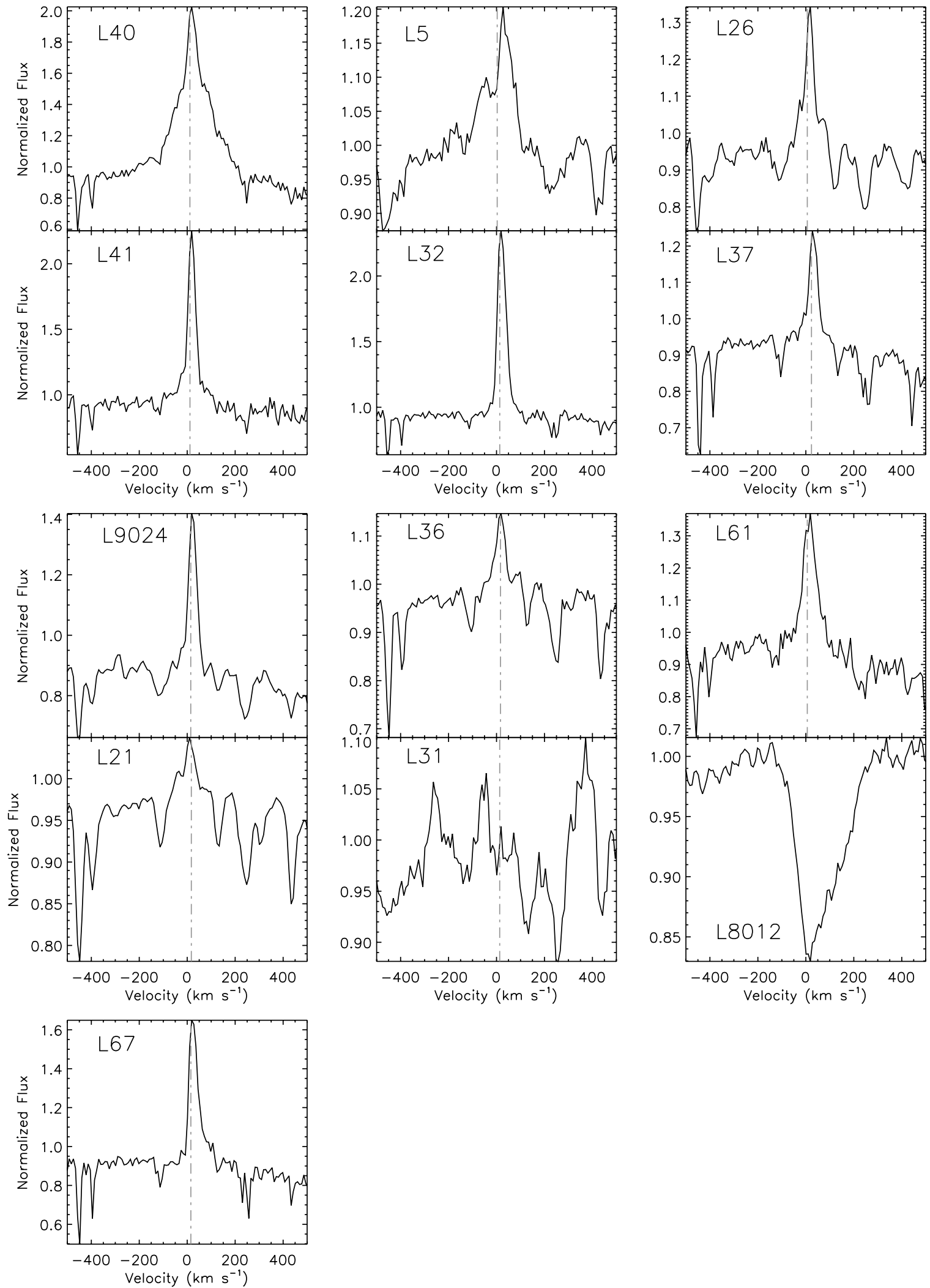

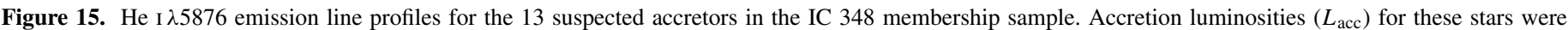

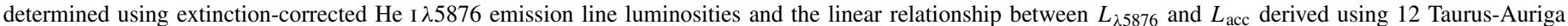

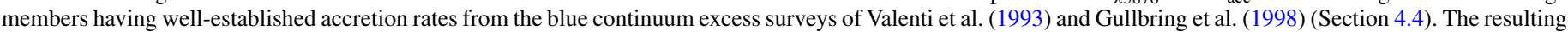

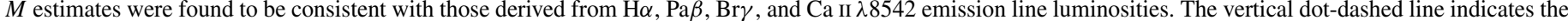
measured radial velocity for each star.

for the near solar mass members of IC 348 , from which $\dot{M}$ follows using the stellar mass and radius estimates from the models of Siess et al. (2000). These $\log \dot{M}$ estimates are given in Table 3 and are found to be comparable to those derived 
from veiling, as well as the $\mathrm{H} \alpha, \operatorname{Pa} \beta$, and $\mathrm{Br} \gamma$ emission line luminosities.

The He I $\lambda 10830$ triplet arises from the $1 \mathrm{~s} 2 \mathrm{~s}^{3} \mathrm{~S}-1 \mathrm{~s} 2 \mathrm{p}^{3} \mathrm{P}^{0}$ transition, the lower level of which is metastable and populated either by collisional excitation from the ground state or by radiative recombination and a subsequent radiative cascade to the $2 \mathrm{~s}^{3}$ level. In the solar spectrum, He I $\lambda 10830$ absorption is believed to be formed when coronal emission shortward of the ionizing potential of $\mathrm{He}$ I (i.e. $\leqslant 504 \AA$ ) impacts the chromosphere and ionizes sufficient numbers of helium atoms to populate the lower level of the $\lambda 10830$ transition. The observed absorption of the solar photospheric continuum is optically thin and the depth of the absorption feature is proportional to the depth and geometry of the chromosphere as well as the strength of the incident coronal emission. In the environment where $\mathrm{He} \mathrm{I} \lambda 10830$ absorption is formed, the electron density is sufficiently low to assume that collisional excitation and deexcitation is unlikely.

Edwards et al. $(2003,2006)$ detect He I $\lambda 10830$ emission in 38 of 39 CTTSs observed in the Taurus-Auriga starforming region. Their high dispersion spectra reveal that most (71\%) CTTSs exhibit blueshifted absorption components of He I $\lambda 10830$, while redshifted absorption components, indicative of magnetospheric accretion flows, are detected in $47 \%$ of the CTTSs in their sample. Among the 39 members of IC 348 observed using SpeX, all suspected accretors exhibit He I $\lambda 10830$ emission as does the non-excess star L11. The $10 \%$ velocity width of $\mathrm{H} \alpha$ for this star is significant $\left(220 \mathrm{~km} \mathrm{~s}^{-1}\right)$, but below the threshold established for accretion $\left(\sim 250 \mathrm{~km} \mathrm{~s}^{-1}\right)$. Given the lack of other accretion signatures, we conclude that L11 was not accreting when observed with HIRES. Shown in Figure 12 are the He I $\lambda 10830$ and Pa $\gamma$ profiles for the 12 accretors (uncorrected for photospheric absorption). The He I $\lambda 10830$ profiles vary dramatically from strong, symmetric emission (e.g., L40, L32), to emission with blue-shifted absorption (e.g., L5, L26), and emission with red-shifted absorption (e.g., L37, L21, L8012B). Several stars exhibit deep subcontinuum absorption that possibly requires an occultation of the star by the disk to reduce continuum strength (Fischer et al. 2007). Lacking high dispersion near-infrared spectra, we are unable to examine the morphology of the He I $\lambda 10830$ line profiles in more detail, but confirm the presence of $\mathrm{He}$ I $\lambda 10830$ emission in even the weakest accretors in the IC 348 sample. Edwards et al. (2003, 2006) have clearly demonstrated the effectiveness of this He I transition as a probe of magnetospheric accretion flows and accretion driven disk winds.

\subsection{Ca II $\lambda 8542$ Emission}

Muzerolle et al. (1998a) examine the Ca II near-infrared triplet $(\lambda \lambda 8498,8542,8662)$ in 11 CTTSs and conclude that their broad emission components are likely formed in the magnetospheric infall region. Their analysis also suggests that the Ca II $\lambda 8542$ emission line flux is a strong correlate of $\dot{M}$ in the absence of blue continuum excess measurements. This correlation persists through four orders of magnitude of $\dot{M}$, making it possible to determine $\dot{M}$ for large numbers of stars using moderate- or high-resolution spectroscopy. We have used ten Taurus-Auriga members examined by Muzerolle et al. (1998a) as well as the four additional Taurus-Auriga stars that were observed with HIRES to derive a linear relationship between $L_{\text {acc }}$ and $L_{\lambda 8542}$. To obtain the emission line fluxes, we follow the procedure outlined by Muzerolle et al. (1998a) and the references therein, subtracting the underlying photospheric absorption spectrum represented by a non-emission star of similar spectral type. The line luminosity is then estimated using the measured equivalent width, the extinction-corrected $I$-band magnitude, the spectral type dependent flux ratio between $\lambda 8542$ and the effective wavelength of the $I$-band filter (obtained from the spectrophotometry of O'Connell 1973), and by assuming a distance of $140 \mathrm{pc}$ to Taurus-Auriga, which is consistent with the spectroscopic parallax measurements of Kenyon et al. (1994). Figure 10(c) plots $L_{\text {acc }}$ as a function of the resulting $L_{\lambda 8542}$ values for the 14 members of Taurus-Auriga. The derived linear regression fit superposed in Figure 10(c) is given by

$\log \left(L_{\mathrm{acc}} / L_{\odot}\right)=(0.94 \pm 0.11) \log \left(L_{\lambda 8542} / L_{\odot}\right)+(2.64 \pm 0.38)$.

Some scatter in the relationship exists, possibly resulting from the non-simultaneous nature of the $\mathrm{Ca}$ II and blue continuum spectrophotometric observations. Two additional Taurus-Auriga members (CW Tau and HN Tau) were excluded from the linear regression analysis because of anomalously large $\mathrm{Ca}$ II $\lambda 8542$ emission line fluxes and correspondingly low measured mass accretion rates. CW Tau is discussed by Muzerolle et al. (1998a) as being earlier than other stars in the sample (K3 spectral type as compared to K7-M0 for the other Taurus members) and a rapid rotator. They also note that a blueshifted absorption component appears at similar velocities for nearly all broad emission lines observed in CW Tau. The Ca II line strength measured in this survey was nearly identical to that measured by Muzerolle et al. (1998a), possibly suggesting that the blue continuum spectrum of Valenti et al. (1993) was obtained during a period of low accretion activity. The line width measured for HN Tau $\mathrm{W}(\lambda 8542)=49.47 \AA$ was the strongest of all Taurus-Auriga members observed with HIRES or by Muzerolle et al. (1998a). The two measurements of $\dot{M}$ found in the literature, however, are similar: $\log \dot{M}=-8.41 M_{\odot} \mathrm{yr}^{-1}$ (Valenti et al. 1993) and $\log \dot{M}=-8.89 M_{\odot} \mathrm{yr}^{-1}$ (Gullbring et al. 1998), suggesting that the HIRES spectrum was obtained during a significant accretion event. The measured equivalent width of $\mathrm{H} \beta$, the one common spectral feature between this survey and the spectrophotometry of Gullbring et al. (1998), however, is significantly less in the HIRES observation: $39.7 \AA$ and $20.5 \AA$ respectively. These discrepancies argue for the need of simultaneous blue spectrophotometry and red moderate resolution spectra to directly examine the relationship between $L_{\text {acc }}$ and $L_{\lambda 8542}$.

Mass accretion rates for the 12 suspected accretors in the IC 348 sample with Ca II $\lambda 8542$ emission present were determined using the above linear relationship and photospheresubtracted, extinction-corrected line fluxes. The resulting log $\dot{M}$ values are given in Table 3 and found to be consistent with those derived from the veiling, $\mathrm{H} \alpha, \mathrm{He}$ I $\lambda 5876, \mathrm{~Pa} \beta$, and $\mathrm{Br} \gamma$ analyses. Shown in Figure 16 are the observed Ca II $\lambda 8542$ emission line profiles before subtraction of the stellar photosphere. Stars having high mass accretion rates (e.g., L40, L5) exhibit very broad emission profiles with velocity widths exceeding $\sim 400 \mathrm{~km} \mathrm{~s}^{-1}$, while others show absorption profiles with core emission reversal (L61, L21, L31). The early-type star L8012B exhibits an asymmetric absorption profile caused by the blend with Pa15. Caution must be observed when using Ca II nearinfrared triplet emission alone as an accretion indicator given that many chromospherically active stars also exhibit significant emission. Among the 19 non-accreting stars in IC 348 that were observed at high resolution, 12 exhibit core emission reversal 

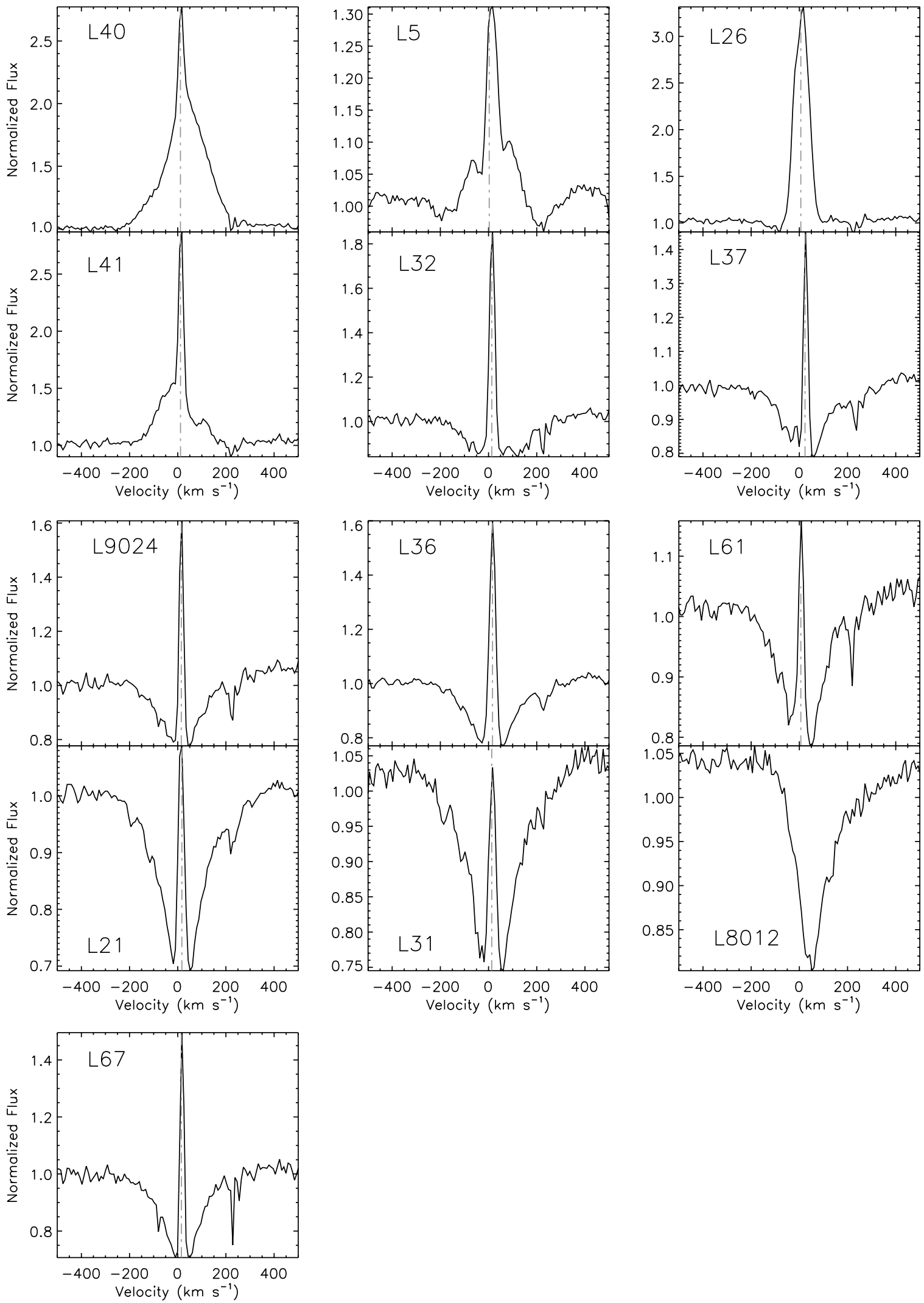

Figure 16. Ca II $\lambda 8542$ emission line profiles for the 13 suspected accretors in the IC 348 membership sample. The asymmetric absorption profile observed for L8012B is caused by the blend of Pa15. The absorption profile accretion luminosities $\left(L_{\text {acc }}\right)$ for these stars were determined using extinction-corrected Ca II $\lambda 8542$ line luminosities $\left(L_{\lambda 8542}\right)$ and the linear relationship between $L_{\lambda 8542}$ and $L_{\text {acc }}$ derived in Section 4.5 . The resulting $\dot{M}$ values were found to be consistent with those derived from $\mathrm{H} \alpha, \mathrm{Pa} \beta, \mathrm{Br} \gamma$, and $\mathrm{He}$ I $\lambda 5876$ emission line luminosities. The vertical dot-dashed line indicates the measured radial velocity for each star.

for all $\mathrm{Ca}$ II near-infrared triplet lines. Without the presence of a broad component of $\mathrm{Ca}$ II $\lambda 8542$ emission, the origin of Ca II emission whether chromospheric or accretion-driven must be carefully reviewed. 


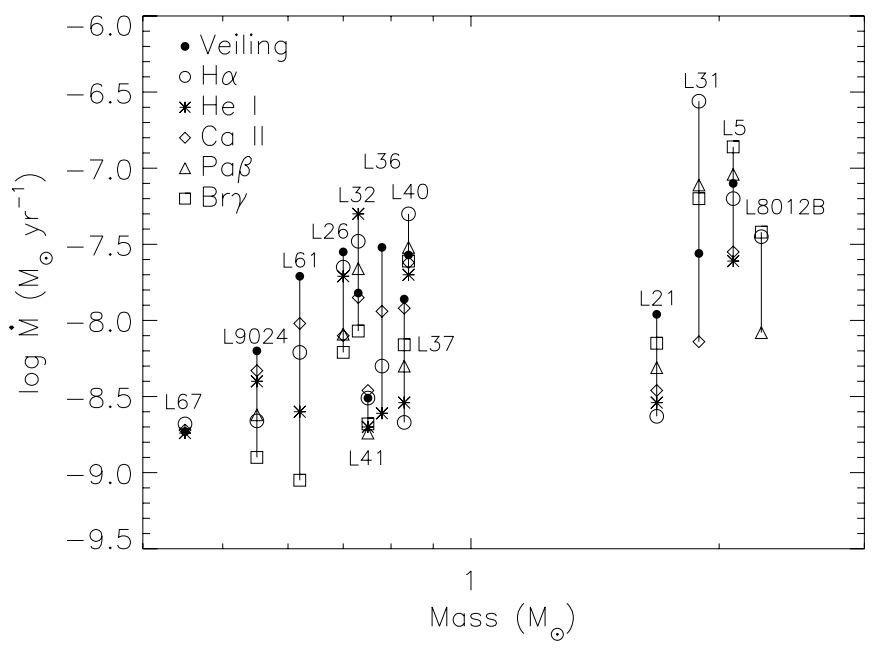

Figure 17. $\log \dot{M}$ plotted as a function of stellar mass from the models of Siess et al. (2000) for the 12 suspected accretors in the IC 348 membership sample. Different symbols are used for each accretion indicator used to estimate $\dot{M}$. For most accretors the dispersion among $\dot{M}$ values is significant, up to and in some cases more than an order of magnitude. Notable exceptions include L40 and L41 for which all six accretion luminosity relationships predict $\log \dot{M}$ values ranging from -7.30 to $-7.70 M_{\odot} \mathrm{yr}^{-1}$ (L40) and from -8.46 to $-8.74 M_{\odot} \mathrm{yr}^{-1}$ (L41). A gap in accreting stars and primordial disk systems is observed extending between masses of $\sim 0.8-1.80 M_{\odot}$. Whether an artifact of extinction uncertainties or spectral-type errors cannot be assessed, but at least a dozen cluster members populate this mass range that do not exhibit signatures of accretion or dust emission.

\subsection{Forbidden Emission}

Cabrit et al. (1990) find that [O I] $\lambda 6300$ emission and $\mathrm{H} \alpha$ emission observed in $23 \mathrm{~T}$ Tauri stars are well correlated and argue that the forbidden emission arises from energetic disk winds and is thus linked with accretion processes. Forbidden emission in the form of [O I] $\lambda 6300$ or [S II] $\lambda \lambda 6717,6731$ is observed in the spectra of all suspected accretors and none of the non-accreting stars in the IC 348 sample (the low signal-to-noise spectrum of L31 exhibits weak [O I] $\lambda 6300$ emission as does the early-type star L8012B). Although the [O I] $\lambda 6300$ feature is impacted by terrestrial water absorption, given the strength of the emission feature in the accretors, the line profile is dominated by the stellar component. [S II] $\lambda \lambda 6717$, 6731 emission is typically associated with jets or bow shocks commonly found in Herbig-Haro objects and outflows (e.g., Reipurth et al. 2002). These emission features are directly linked with accretion processes and their detection in the highresolution spectra of IC 348 members is used as confirmation of accretion activity. Observed forbidden transitions are listed in Table 1.

\section{DISCUSSION}

The derived mass accretion rates for the 13 suspected accretors in the IC 348 membership sample are summarized in Table 3 for all diagnostics examined. For most stars the scatter among $\dot{M}$ values is significant, up to and, in some cases, more than an order of magnitude (L31, L61). Notable exceptions include L40 and L41 for which all six accretion luminosity relationships predict $\log \dot{M}$ values ranging from -7.30 to $-7.70 M_{\odot} \mathrm{yr}^{-1}$ (L40) and from -8.46 to $-8.74 M_{\odot} \mathrm{yr}^{-1}$ (L41). Figure 17 demonstrates the observed dispersion in mass accretion rates, plotting $\log \dot{M}$ from all accretion diagnostics examined as a function of stellar mass from the models of Siess et al. (2000). Excluding the early-type binary L8012B, $\mathrm{Pa} \beta$, and $\mathrm{Br} \gamma$ emission luminosities consistently yield comparable $\log \dot{M}$ values. For embedded sources, $\mathrm{Pa} \beta$ and $\mathrm{Br} \gamma$ emission likely produce the most reliable estimates of $\dot{M}$ given the significantly reduced impact of extinction in the near-infrared. L31 exemplifies this circumstance with $A_{V} \sim 12$ mag and highly disparate $\dot{M}$ determinations from $\mathrm{H} \alpha$ and $\mathrm{Ca}$ II $\lambda 8542$ emission. The $\mathrm{Pa} \beta$ and $\mathrm{Br} \gamma \dot{M}$ values, however, are virtually identical. Further supporting the use of $\mathrm{Pa} \beta$ and $\mathrm{Br} \gamma$ emission to derive $L_{\text {acc }}$, we find that only suspected accretors in the IC 348 membership sample exhibit these lines in emission. Several non-accreting and non-infrared excess members of IC 348, however, are observed with weak $\mathrm{H} \alpha, \mathrm{He}$ I, and $\mathrm{Ca}$ II emission, presumably arising from enhanced chromospheric activity.

One notable feature in Figure 17 is the apparent gap in accreting stars and primordial disk candidates in IC 348 that extends from $\sim 0.84 M_{\odot}$ to $\sim 1.80 M_{\odot}$. This gap is also apparent in Figure 4, the extinction-corrected, CMD of the cluster membership sample. There are a number of potential explanations including extinction errors, uncertainties in spectral typing, and sample incompleteness. More than a dozen cluster members populate this mass range, none of which were identified as possible accretors or as primordial disk candidates by Lada et al. (2006). Whether this apparent gap is a real effect or an artifact of observational uncertaintites cannot be adequately addressed here. Of the 11 cluster members in the Luhman et al. (2003) stellar census earlier than G0 (L1-4, L7-8, L10, L17, L19, L25, L30), L2, L8 (both components), and L19 were observed with HIRES on 2008 November 29. Weak core emission reversal is apparent in the $\mathrm{H} \alpha$ profile of $\mathrm{L} 2$, an A2-type primordial disk candidate, while stronger emission is evident in the $\mathrm{H} \alpha$ profile of L19, another A2-type primordial disk candidate. No forbidden line emission (e.g. [O I] $\lambda 6300$ or [S II] $\lambda \lambda 6717,6731$ ), however, was detected in the spectrum of either of these stars. Both components of the A2-type, weak-disk candidate L8 exhibit pure absorption $\mathrm{H} \alpha$ profiles. With the exception of L17, all other early-type IC 348 members were observed with WFGS either by Herbig (1998) or by Dahm, and none exhibit detectable $\mathrm{H} \alpha$ emission (the limiting measurable equivalent width of WFGS is $\sim 3 \AA$ ). It is therefore possible that the intermediate mass (1.8$2.3 M_{\odot}$ ) stars shown in Figure 17 are the most massive accretors remaining in the cluster.

To characterize disk emission among all IC 348 members, Lada et al. (2006) use the slope of the stellar SED through the four IRAC channels, $\alpha$. By comparing the observed SEDs with radiative transfer disk models that assume a $\Sigma \sim r^{-1}$ surface density profile, they conclude that stars best matching the circumstellar disk models are represented by slopes of $\alpha \geqslant-1.80$. These are assumed to be primordial, optically thick disk candidates. Pure stellar photospheres are characterized by the SED of an M0-type star, which including a margin of uncertainty has a slope $\alpha \leqslant-2.56$. Weak or transition disk systems are then represented by SED slopes that range between these two extrema, $-2.56 \leqslant \alpha<-1.80$. Lada et al. (2006) find that $68 \%$ of CTTSs in IC 348 are associated with primordial disks and that $11 \%$ exhibit weak or optically thin disk emission. Among WTTSs, $12 \%$ are found with optically thick disks while $22 \%$ exhibit weak disk emission. From these statistics, Lada et al. (2006) conclude that the presence of hot dust emission and gaseous accretion are strongly coupled. The distinction between CTTSs and WTTSs in the Lada et al. (2006) survey, however, is based solely upon the equivalent width of $\mathrm{H} \alpha$, which, as noted in Section 4.2, is not capable of distinguishing weakly accreting systems and may confuse strong chromospheric emission found in late-type stars for evidence of accretion activity. Shown 


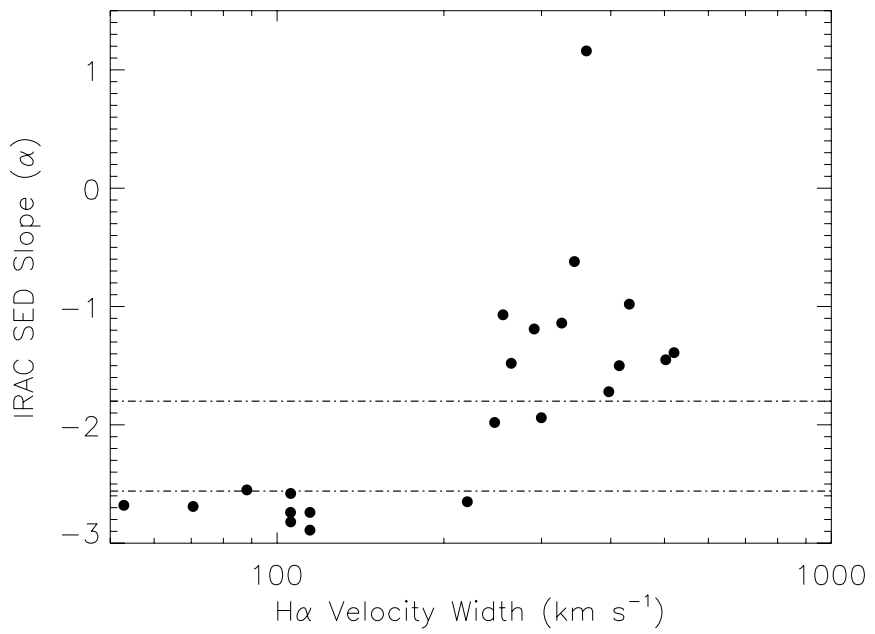

Figure 18. The IRAC slope parameter $(\alpha)$ from Lada et al. (2006) plotted as a function of the velocity width of $\mathrm{H} \alpha$ (measured at $10 \%$ peak flux) for all stars in the IC 348 membership sample with observed $\mathrm{H} \alpha$ emission (11 members exhibit $\mathrm{H} \alpha$ absorption). The accretors in the sample are well separated from those stars with purely chromospheric emission. The dot-dashed lines mark the boundaries of the various classes of disk structure using the Lada et al. (2006) criteria (primordial disks: $\alpha \geqslant-1.80$, weak: $-2.56 \leqslant \alpha<-1.80$, non-excess: $\alpha<-2.56)$. The strong correlation between gaseous accretion and dust emission is evident.

in Figure 18 is the IRAC SED slope $(\alpha)$ from Lada et al. (2006) plotted as a function of $\mathrm{H} \alpha$ velocity width at $10 \%$ peak flux for all stars in the IC 348 membership sample having detected $\mathrm{H} \alpha$ emission (11 stars in the sample exhibit absorption profiles). The 13 suspected accretors are clearly separated from the non-accreting stars whose $\mathrm{H} \alpha$ emission likely arises from enhanced chromospheric activity. L11 is the single non-disk candidate exhibiting a significant $\left(220 \mathrm{~km} \mathrm{~s}^{-1}\right)$ velocity width
(Section 4.3). The strong coupling between dust emission as evidenced by the slope of the IRAC SED and gaseous accretion is readily apparent.

Longer wavelength emission may provide a better probe of disk settling effects and is also potentially sensitive to changes in mass accretion rate (D'Alessio et al. 2006). Lacking mid-infrared spectra for these stars we use available $24 \mu \mathrm{m}$ photometry from Lada et al. (2006) to sample disk emission beyond the IRAC passbands. Shown in the left panel of Figure 19 is the SED slope from 8.0 to $24.0 \mu \mathrm{m}$ plotted against the IRAC 3.6 to $8.0 \mu \mathrm{m}$ slope for 12 suspected accretors and 5 additional near solar mass stars with $24 \mu \mathrm{m}$ detections. While the nonexcess stars are clearly separated from the disk candidates, a further gradation is apparent among the suspected accretors. Five of these stars exhibit significantly rising slopes beyond $8.0 \mu \mathrm{m}$, two of which are transition or weak disk systems (L67 and L21). In the right panel of Figure 19, $\log M$ is plotted as a function of the IRAC 3.6-8.0 $\mu \mathrm{m}$ slope. The five suspected accretors with significant $24 \mu \mathrm{m}$ excesses span the full range of observed accretion rates. No statistically significant correlation is evident between $\dot{M}$ and $\alpha$. Similarly, no correlation is found between $\dot{M}$ and the 8.0-24.0 $\mu \mathrm{m}$ slope; however, the small number of stars examined here do not allow broader conclusions to be drawn.

The 40 stars observed with HIRES, SpeX, or both instruments constitute approximately $87 \%$ of the total cluster population having spectral types from G0 to M0 $\left(\sim 2.0-0.5 M_{\odot}\right)$. All but one primordial disk candidate within this range were observed (the single exception being the faint $K_{S}=13.2$ M0-type star L276). The remaining unobserved stars (L113, L191, L105, L142, L144) are non-infrared excess stars having spectral types of K6, K7, and M0 (for the latter 3), respectively. With the exception of L9060, for which only a low SN SpeX spectrum could be obtained, all primordial disk candidates
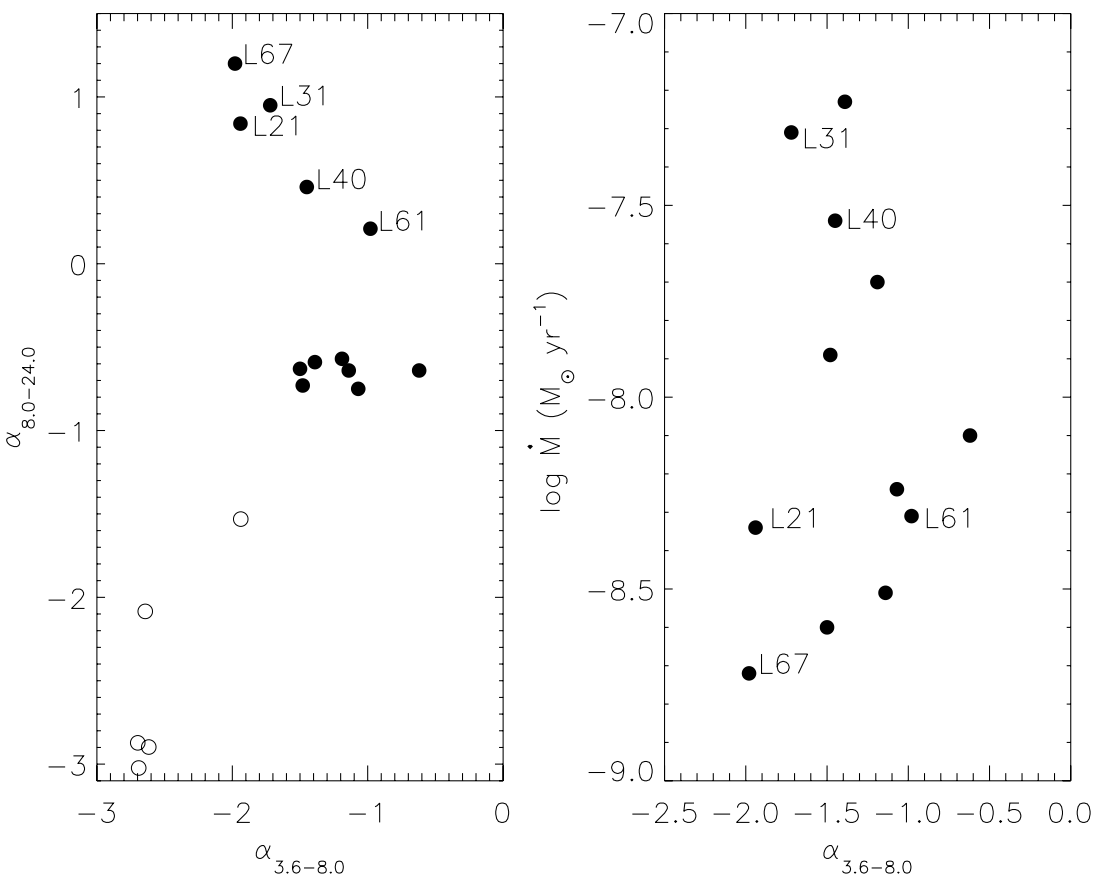

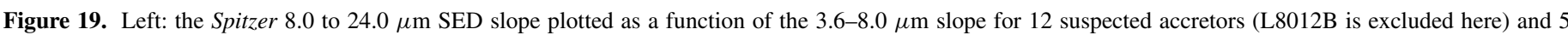

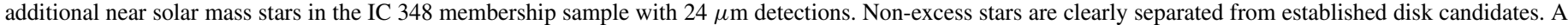

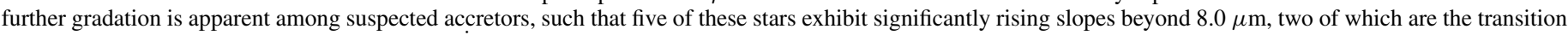

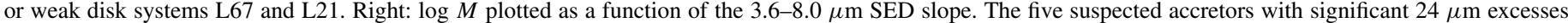

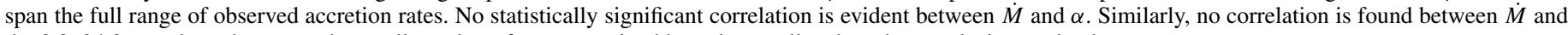
the 8.0-24.0 $\mu \mathrm{m}$ slope; however, the small number of stars examined here do not allow broader conclusions to be drawn. 

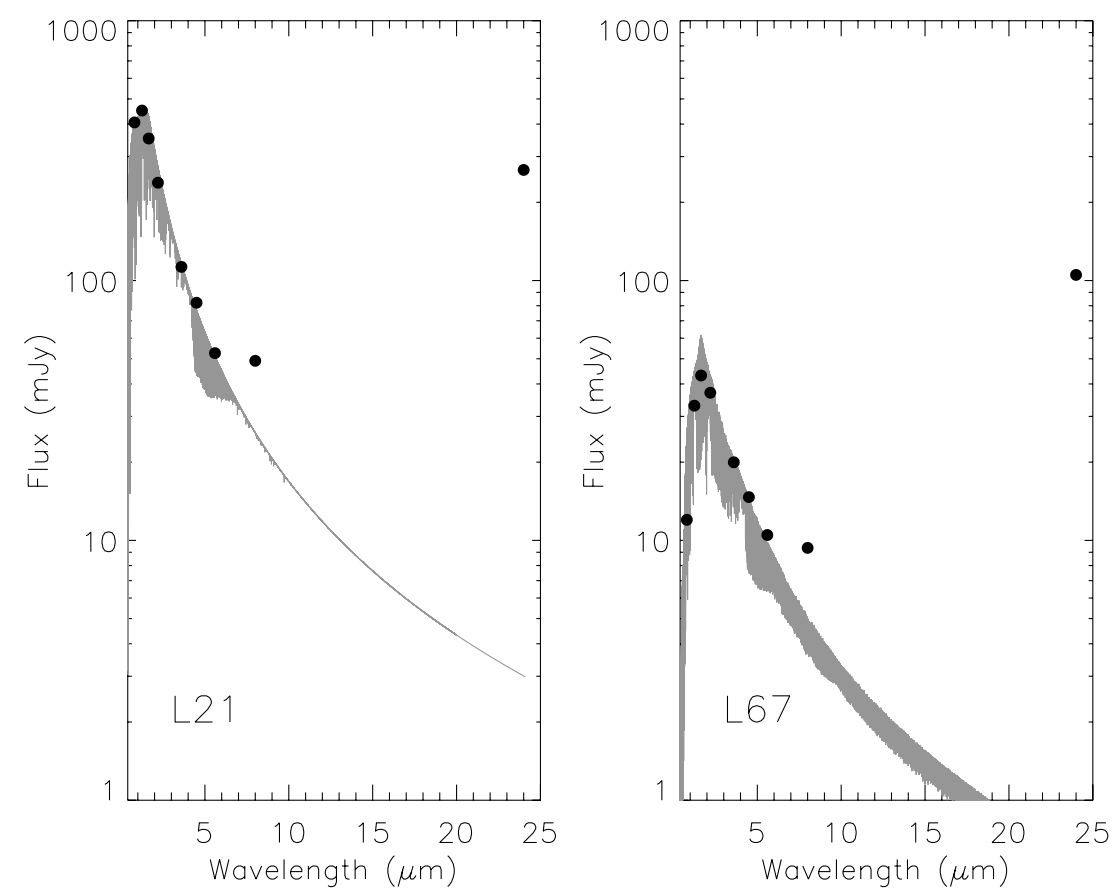

Figure 20. The SEDs for two weak or transition disk candidates suspected of accretion, L21 and L67. The NextGen stellar atmospheric models of Hauschildt et al. (1999) for solar metalicities and subgiant surface gravities are superposed for effective temperatures of $5200 \mathrm{~K}$ (K0) and 3800 K (M0), respectively. The SEDs of L21 and L67 clearly exhibit near-photospheric profiles out to $\sim 4.5 \mu \mathrm{m}$, beyond which the Spitzer photometry deviates dramatically from the stellar atmospheric models. The mid-infrared photometry confirms the presence of opacity gaps within the inner regions of these disks.

examined exhibit evidence for magnetospheric accretion. Among non-infrared excess stars, none are found with the broadened $\mathrm{H} \alpha$ emission profiles or with forbidden line emission characteristic of accretion processes. The immediate implication is that the presence of inner disk gas and dust are strongly correlated within optically thick disks. If the Luhman et al. (2003) census represents a complete cluster membership sample of IC 348 within this range of spectral types, then $27 \pm 8 \%$ exhibit primordial disk emission, $9 \% \pm 4 \%$ retain optically thin disk emission, and the remaining $64 \%$ are non-excess stars that have already cleared micron-sized dust to orbital radii of at least $\sim 5 \mathrm{AU}$. The accretion fraction within this mass range is comparable to the primordial disk fraction. Marcy et al. (2005) find that of 1330 FGKM-type field stars examined by high-precision radial velocity surveys, $6.6 \%$ host giant planets orbiting within $5 \mathrm{AU}$. Extrapolating this lower limit, they conclude that $\sim 12 \%$ of all FGK-type stars possess a giant planet within $20 \mathrm{AU}$. While strongly metalicity dependent, this fraction of stars hosting planetary systems is a factor of 2 less than the accretion fraction found in IC 348. Sufficient numbers of gas-rich, optically thick disks therefore remain at ages of 2-3 Myr to account for the observed fraction of exoplanets in low and intermediate mass field stars.

Of the five weak or transition disk candidates examined in the survey-L6 (G3), L21 (K0), L8024 (K7), L65 (M0), and L67 (M0.5) - two exhibit definitive signatures of accretion, L21 and L67. The G3-type transition disk candidate, L6, showed no indication of $\mathrm{H} \alpha$ emission either during Herbig's (1998) survey or the HIRES survey, but its photosphere-subtracted nearinfrared spectrum reveals weak $\mathrm{Ca}$ II, $\mathrm{Pa} \beta$, and $\mathrm{Br} \gamma$ emission. It is possible that the non-excess template stars adopted for L6 improperly correct for photospheric absorption. Supporting this notion, the HIRES spectrum of L6 reveals no indication of core emission reversal for the Ca II near-infrared triplet lines.
The transition disk status of L65 is also unclear given that its purported $8 \mu \mathrm{m}$ excess is not statistically robust and that only an upper limit at $24 \mu \mathrm{m}$ is given by Lada et al. (2006).

The SEDs for L21 and L67 are reproduced in Figure 20 using extinction-corrected, BVI-band, 2MASS, and Spitzer IRAC and MIPS photometry (from Lada et al. 2006). Superposed in the figure are the NextGen stellar atmospheric models of Hauschildt et al. (1999), with effective temperatures of 5200 $(\mathrm{K} 0)$ and $3800 \mathrm{~K}(\mathrm{M} 0)$ and $\log (g)=4.0$. The SEDs of L21 and L67 clearly exhibit near-photospheric profiles out to $\sim 4.5 \mu \mathrm{m}$, beyond which the Spitzer photometry deviates dramatically. The optically thin interiors of these weak or transition disks may suggest that significant evolution has occurred, with micronsized dust grains being removed from the terrestrial region. Several physical mechanisms responsible for inner disk clearing have been proposed, including: (1) dust grain growth and mid-plane settling which would deplete the inner disk of micronsized grains leaving a gaseous inner disk that continues to accrete onto the stellar photosphere; (2) the formation of a giant planet that dynamically clears a gap within the disk, isolating the inner and outer disk regions (as gas depletes within the inner disk $\dot{M}$ is expected to decrease by a factor of 10 ; Najita et al. 2007); (3) photoevaporation of the disk by stellar UV and FUV flux. Transition disks resulting from photoevaporative flows are distinguishable from other evolutionary pathways by their expected low mass accretion rates $\left(\sim 10^{-10} M_{\odot} \mathrm{yr}^{-1}\right)$ and low disk masses (Alexander et al. 2006; Najita et al. 2007). The moderate mass accretion rates estimated for L21 and L67, $\log \dot{M} \sim-8.34$ and $-8.72 M_{\odot} \mathrm{yr}^{-1}$, respectively, can only exclude photoevaporation as a dominant clearing mechanism. Giant planet formation and dust grain growth remain viable possibilities. L21 is also a wide binary having a companion lying $\sim 8^{\prime \prime}$ to the northwest. Whether this star is physically associated with $\mathrm{L} 21$ cannot be assessed, but given a projected separation of 
$\sim 2600$ AU, its impact upon inner disk evolution is not expected to be significant. Additional observations of weak or transition disk candidates in IC 348 are needed to evaluate disk masses, structure, and composition to further constrain the mechanisms responsible for inner disk clearing.

\section{CONCLUSIONS}

The principal results from this investigation are as follows.

1. High-resolution optical and moderate-resolution nearinfrared spectra of 40 near solar mass $\left(\sim 2.0-0.5 M_{\odot}\right)$ members of the 2-3 Myr old cluster IC 348 were obtained in order to examine established accretion diagnostics and the coupling between inner disk gas and hot dust emission inferred from Spitzer derived infrared excesses. The membership sample was drawn from the stellar census of Luhman et al. (2003) with cluster membership being confirmed by radial velocity analysis and the presence of $\mathrm{Li}$ I $\lambda 6708 \mathrm{ab}-$ sorption. Modifying the $\mathrm{H} \alpha 10 \%$ peak flux velocity width criterion of White \& Basri (2003) to $250 \mathrm{~km} \mathrm{~s}^{-1}$, we find 13 suspected accretors in the IC 348 membership sample, all of which exhibit forbidden emission ([O I $] \lambda 6300$ and $[\mathrm{S}$ II $] \lambda 6731$ ) generally associated with accretion driven winds. All but one primordial disk candidate and two (of five) weak or transition disk candidates in the IC 348 membership sample are found to be accreting. The immediate implication is that inner disk gas and hot, micron-sized dust emission inferred from thermal and mid-infrared excesses are strongly coupled within this range of stellar masses. Given the restricted sample size, however, these results are not necessarily representative of the cluster population as a whole. Additional spectroscopic observations of less massive disk candidates in IC 348 are presently underway.

2. Mass accretion rates for the 13 suspected accretors have been derived using estimates for veiling at $\lambda 6500$ as well as five independent linear relationships between accretion luminosity and emission line flux for $\mathrm{H} \alpha$, $\mathrm{He}$ I $\lambda 5876$, $\mathrm{Ca}$ II $\lambda 8542, \mathrm{~Pa} \beta$, and $\mathrm{Br} \gamma$. The resulting accretion luminosities are then transformed to $\dot{M}$ using the stellar mass and radius estimates of the Siess et al. (2000) pre-mainsequence models. The derived $\dot{M}$ values range from $\log$ $\dot{M}=-8.7$ to $-7.2 M_{\odot} \mathrm{yr}^{-1}$, with a median value for the accreting population of $-8.1 M_{\odot} \mathrm{yr}^{-1}$. For most suspected accretors, the scatter among individual $\log \dot{M}$ estimates is significant, up to and in some cases more than an order of magnitude. Notable exceptions include L40 and L41 for which all six accretion luminosity relationships predict $\log \dot{M}$ values ranging from -7.30 to $-7.70 M_{\odot} \mathrm{yr}^{-1}$ (L40) and from -8.46 to $-8.74 M_{\odot} \mathrm{yr}^{-1}$ (L41). $\mathrm{Pa} \beta$ and $\mathrm{Br} \gamma$ emission are detected only among accreting stars in the IC 348 membership sample, implying their sensitivity to accretion processes. Higher-order Brackett lines (Br 10-13) at $H$-band are only observed in emission for the strongly accreting star L40. For heavily extincted sources, $L_{\mathrm{Pa} \beta}$ and $L_{\mathrm{Br} \gamma}$ likely provide the most reliable estimates of $\dot{M}$ given the significantly reduced impact of extinction in the near-infrared.

3. Comparing observed $\mathrm{H} \alpha$ emission line fluxes and derived mass accretion rates for nine suspected accretors in IC 348 with the magnetospheric accretion models of Muzerolle et al. (1998b) and Kurosawa et al. (2006), we find that gas temperatures of $\sim 10,000 \mathrm{~K}$ are predicted by both models. These gas temperatures are near the upper limit derived by
Muzerolle et al. (1998b) before continuum emission becomes optically thick and effectively veils emission arising from the accretion column. The Muzerolle et al. (1998b) models for $\mathrm{Pa} \beta$ and $\mathrm{Br} \gamma$ emission agree well with observed line fluxes and derived $\dot{M}$ estimates, possibly resulting from the reduced extinction effects at $J$ - and $K$-bands $\left(\sim 0.28 A_{V}\right.$ and $0.12 A_{V}$, respectively). Incorporating other $\mathrm{H}$ I emission lines (e.g. Balmer, Paschen, Brackett series) and ion species (e.g., He I, Ca II, Na I) into future radiative transfer models of magnetospheric accretion should provide better constraints for the physical conditions present within the accretion columns and the impact point on the stellar photosphere.

4. Edwards et al. $(2003,2006)$ clearly demonstrate the effectiveness of He I $\lambda 10830$ as a probe of magnetospheric accretion flows and accretion driven disk winds. Among the 39 members of IC 348 observed with SpeX, all 12 suspected accretors exhibit He I $\lambda 10830$ emission as does the non-infrared excess star L11. The He I $\lambda 10830$ profiles vary dramatically from strong, symmetric emission, to emission with blue-shifted and red-shifted absorption. Several stars exhibit deep subcontinuum absorption profiles that are not understood, but high dispersion near-infrared spectra are needed to examine the morphology of the He I $\lambda 10830$ line profiles in more detail.

5. The 3.6-8.0 $\mu \mathrm{m}$ SED slope $(\alpha)$ and the $\mathrm{H} \alpha 10 \%$ peak flux velocity width accretion criterion of White \& Basri (2003) appear to be powerful diagnostics of hot dust emission and gaseous accretion, respectively. Plotting $\alpha$ against the $\mathrm{H} \alpha$ velocity width clearly separates the suspected accretors from those stars with purely chromospheric $\mathrm{H} \alpha$ emission. We find no statistically significant correlation between $\dot{M}$ and the 3.6-8.0 $\mu \mathrm{m}$ or the 8.0-24.0 $\mu \mathrm{m}$ SED slope, although the small number of stars examined does not allow broader conclusions to be drawn.

6. Of the five weak or transition disk candidates examined in the survey-L6 (G3), L21 (K0), L8024 (K7), L65 (M0), and L67 (M0.75) — only two exhibit definitive signatures of accretion, L21 and L67. The SEDs of these stars clearly exhibit near-photospheric profiles out to $\sim 4.5 \mu \mathrm{m}$, suggesting that significant disk evolution has occurred, with micron-sized dust grains being removed from the terrestrial region. The derived mass accretion rates for these stars, log $\dot{M} \sim-8.34 M_{\odot} \mathrm{yr}^{-1}$ and $-8.72 M_{\odot} \mathrm{yr}^{-1}$, respectively, can effectively exclude photoevaporation as the dominant clearing mechanism given the expected low $\dot{M}$ predicted for such evolved disks, $\log \dot{M} \sim-10 M_{\odot} \mathrm{yr}^{-1}$.

S.E.D. is supported by an NSF Astronomy and Astrophysics Postdoctoral Fellowship under award AST-0502381. Some of the data presented herein were obtained at the W. M. Keck Observatory, which is operated as a scientific partnership among the California Institute of Technology, the University of California, and the National Aeronautics and Space Administration. The Observatory was made possible by the generous financial support of the W. M. Keck Foundation. We have made use of the SIMBAD database operated at CDS, Strasbourg, France, and the Two Micron All Sky Survey (2MASS), a joint project of the University of Massachusetts and the Infrared Processing and Analysis Center (IPAC)/California Institute of Technology, funded by NASA and the National Science Foundation. S.E.D. gratefully acknowledges Lynne Hillenbrand for support and helpful discussions as well as Suzan Edwards and Will 


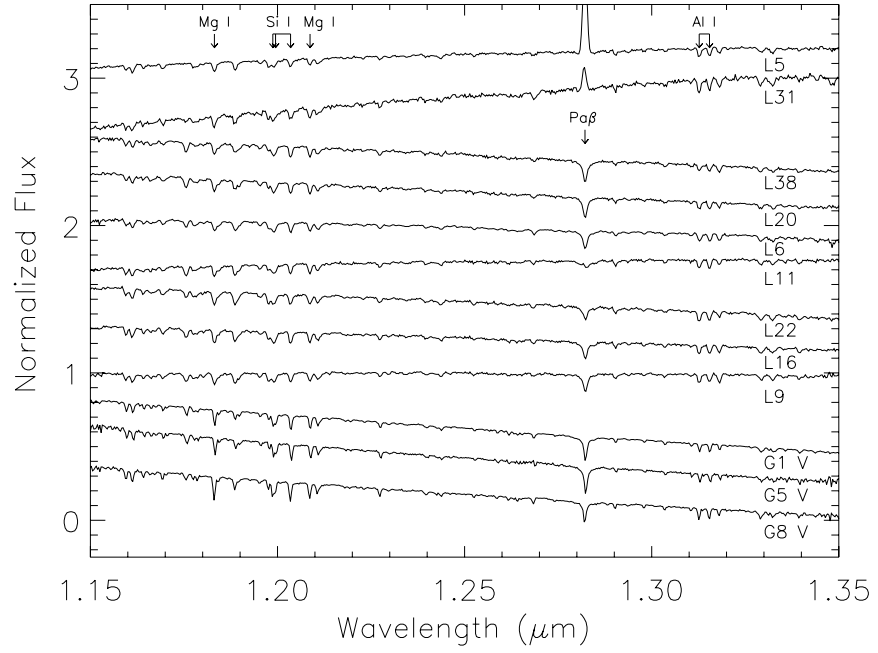

Figure 21. SpeX $J$-band spectra for the G-type stars in the IC 348 membership sample.

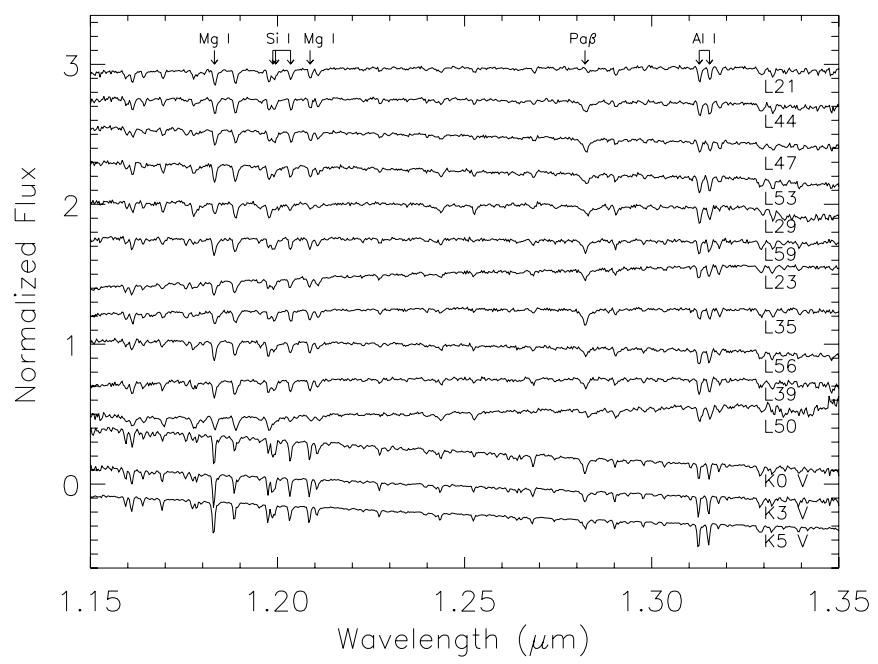

Figure 22. SpeX $J$-band spectra for the K-type stars in the IC 348 membership sample.

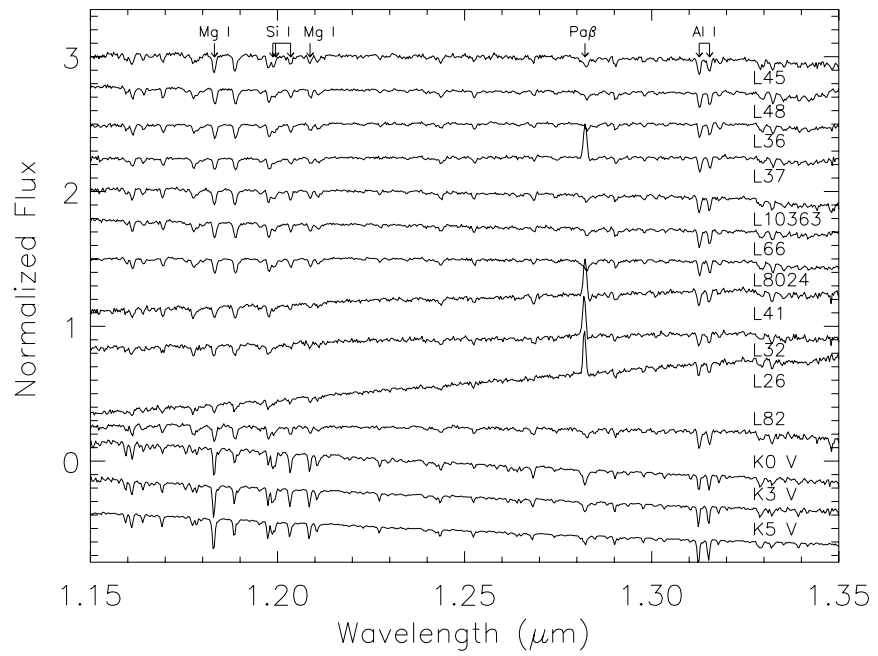

Figure 23. SpeX $J$-band spectra for the K-type stars in the IC 348 membership sample.

Fischer for use of several HIRES observations of Taurus members that proved invaluable for this survey. S.E.D. also thanks an anonymous referee whose constructive comments signifi-

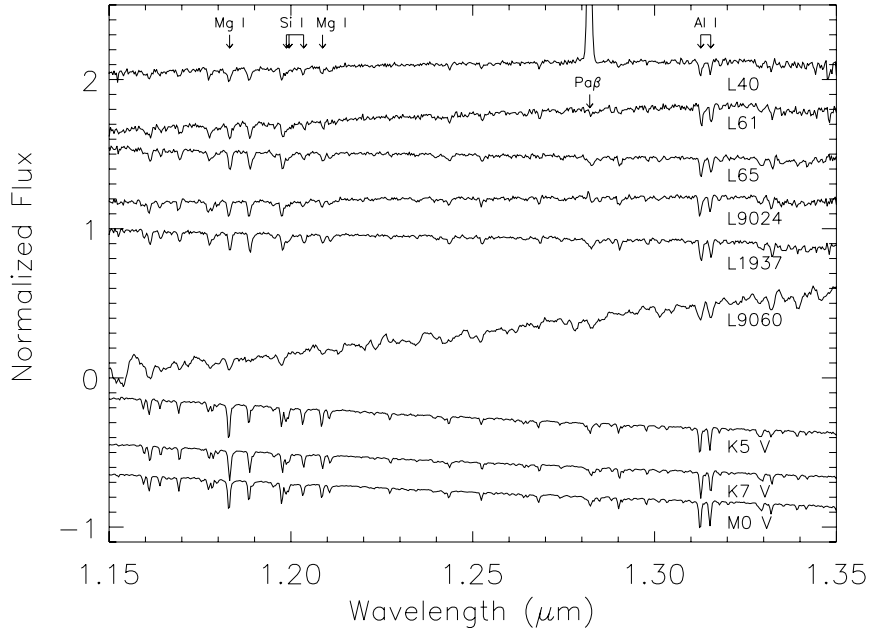

Figure 24. SpeX $J$-band spectra for the M-type stars in the IC 348 membership sample.

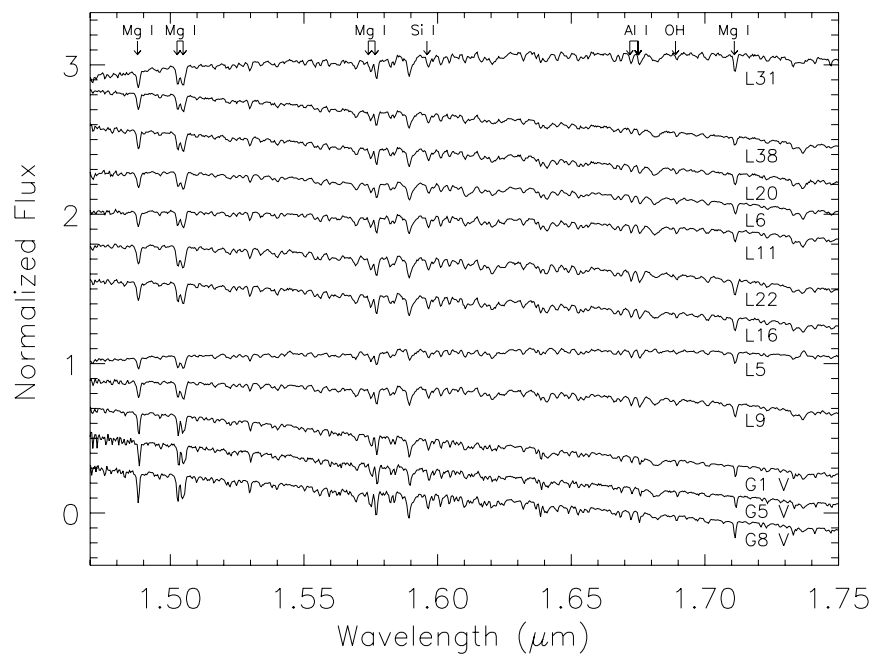

Figure 25. SpeX $H$-band spectra for the G-type stars in the IC 348 membership sample.

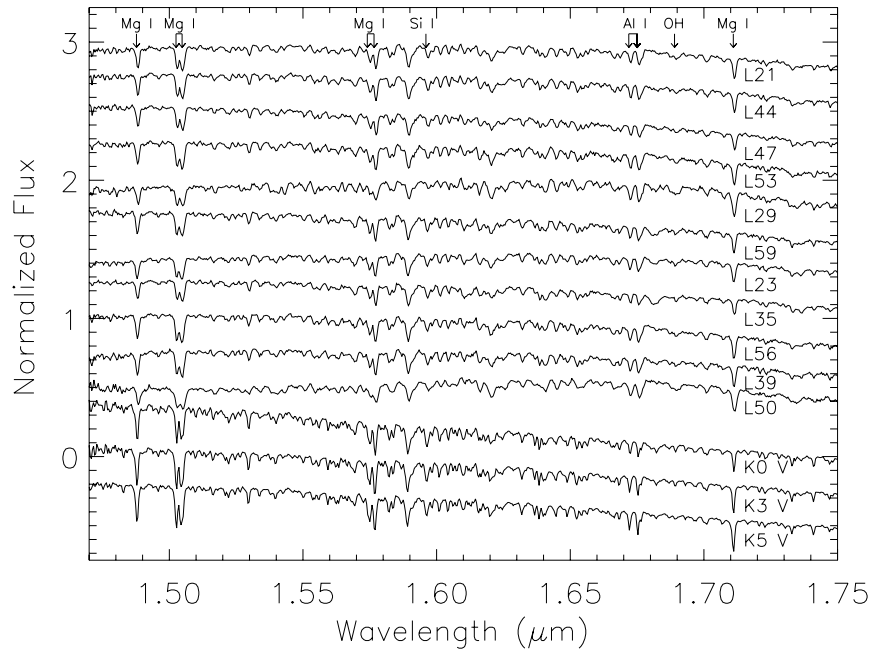

Figure 26. SpeX $H$-band spectra for the K-type stars in the IC 348 membership sample.

cantly improved this paper. A special debt of gratitude goes out to Tom Barlow who wrote, and Patrick Shopbell who implemented minor patches to, the makee HIRES reduction software 


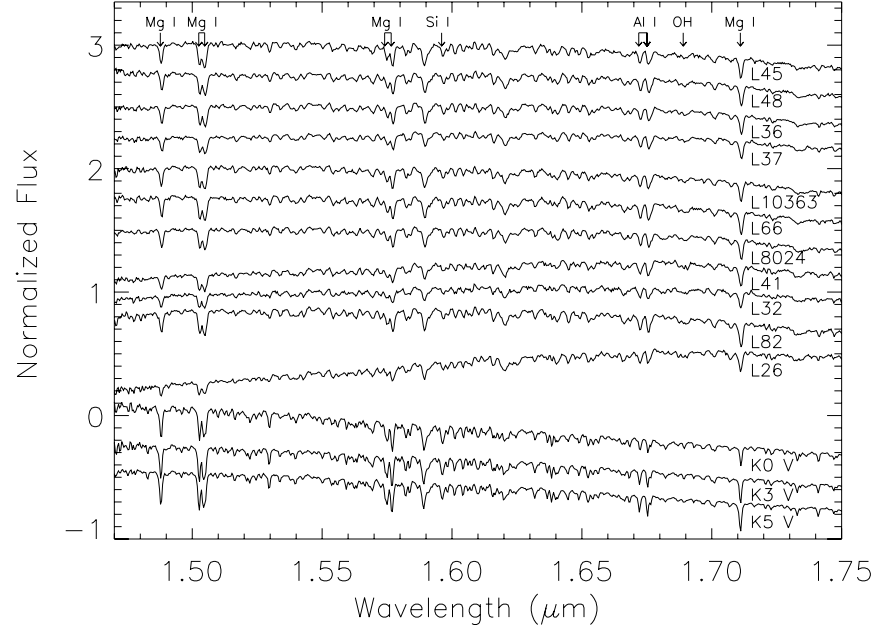

Figure 27. SpeX $H$-band spectra for the K-type stars in the IC 348 membership sample.

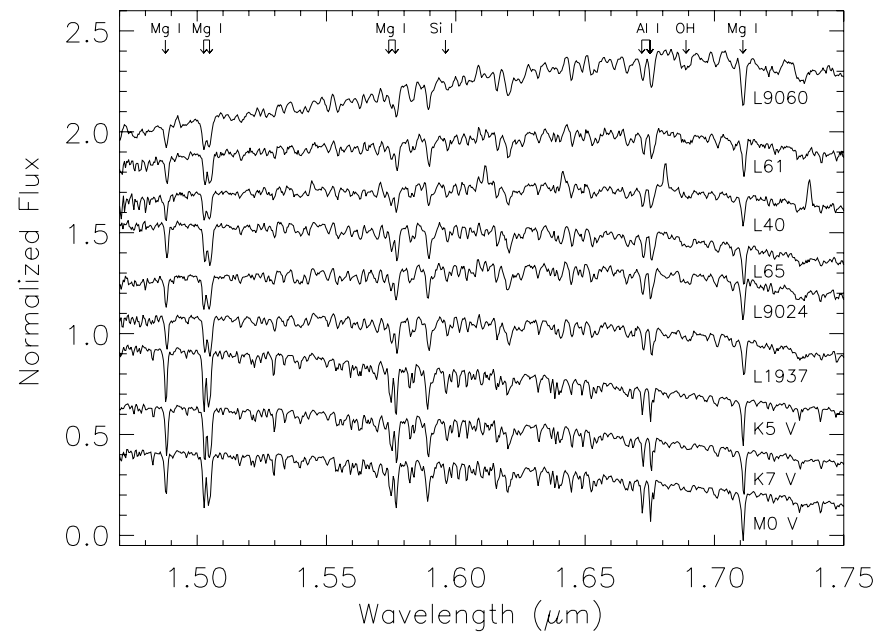

Figure 28. SpeX $H$-band spectra for the M-type stars in the IC 348 membership sample.

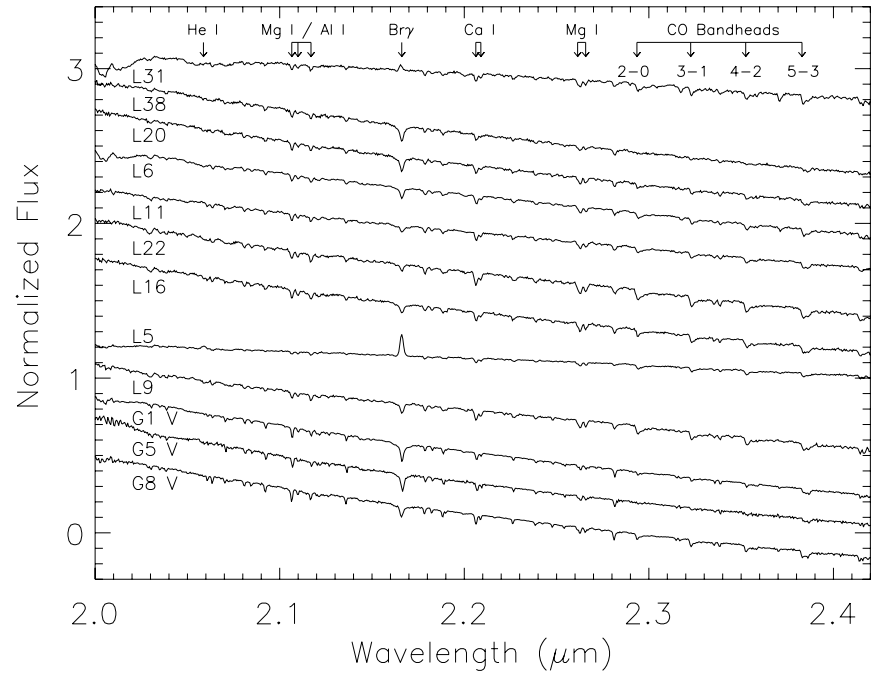

Figure 29. SpeX $K$-band spectra for the G-type stars in the IC 348 membership sample.

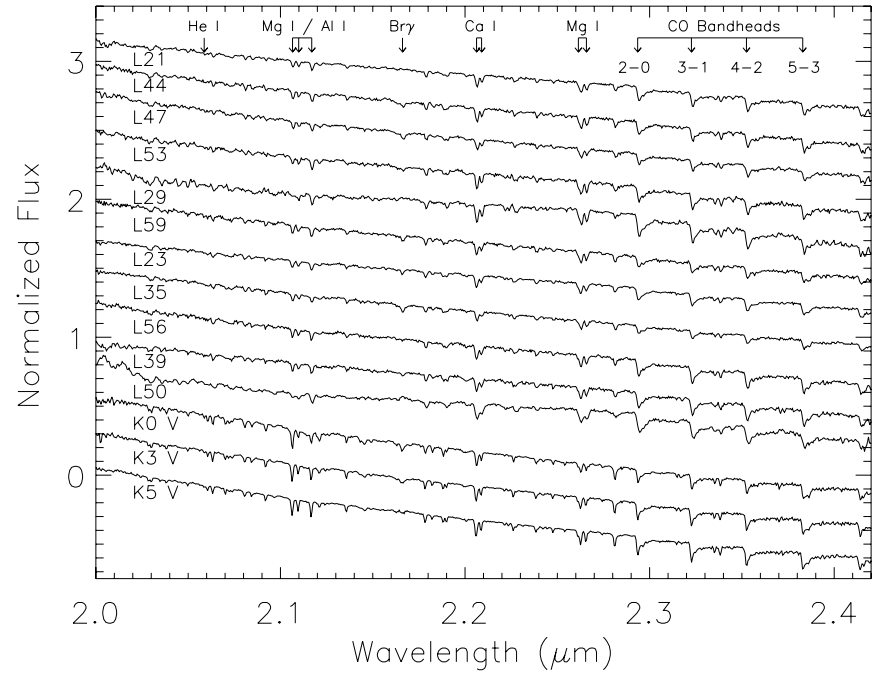

Figure 30. SpeX $K$-band spectra for the K-type stars in the IC 348 membership sample.

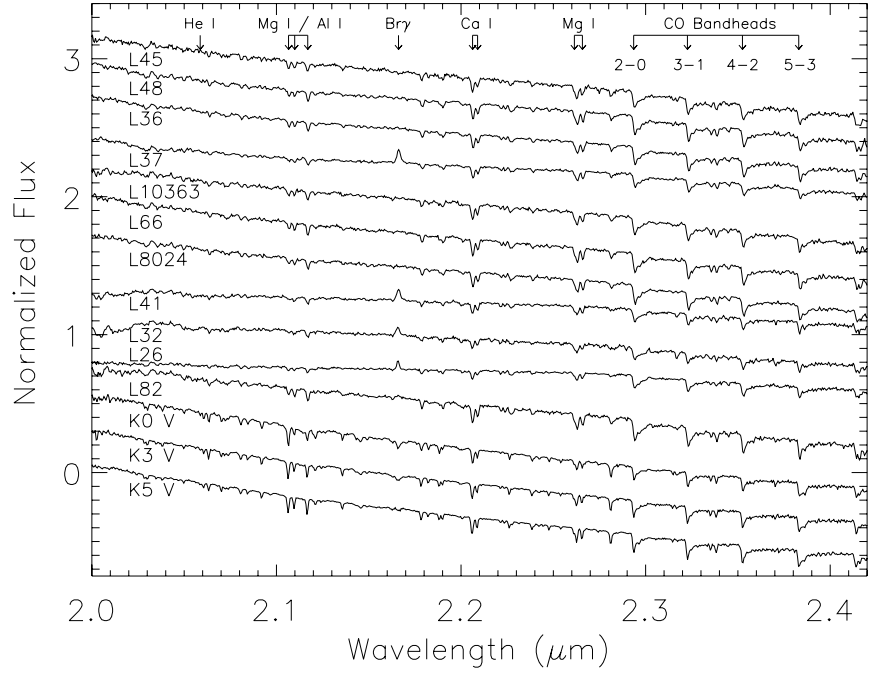

Figure 31. SpeX $K$-band spectra for the K-type stars in the IC 348 membership sample.

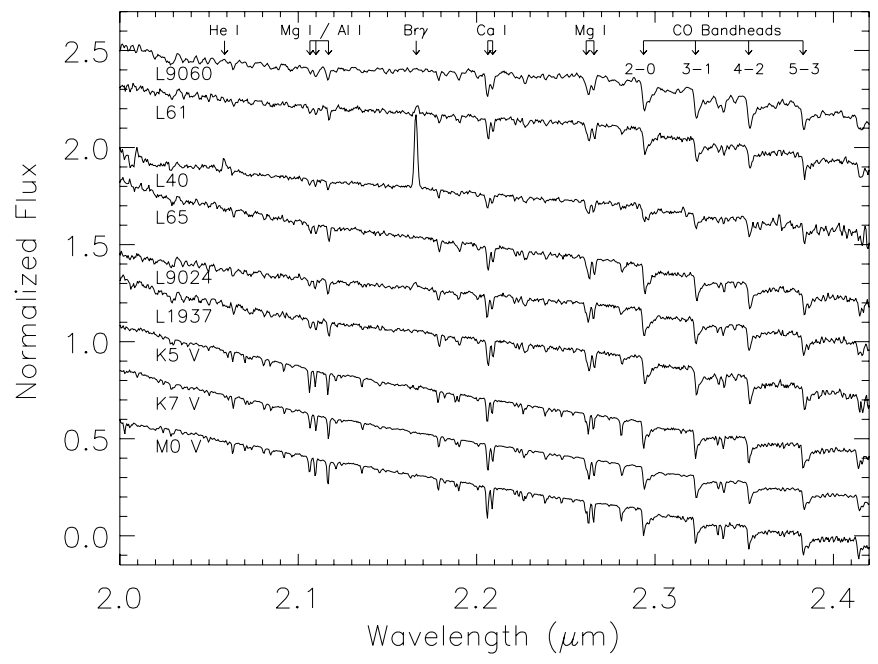

Figure 32. SpeX $K$-band spectra for the M-type stars in the IC 348 membership sample. 
package allowing its use for the cross-disperser and echelle angles adopted for this survey.

\section{APPENDIX}

The $J H K$-SpeX spectra for 36 IC 348 members are reproduced in Figures $19-22$ ( $J$-band), Figures $23-26$ ( $H$-band), and Figures 27-30 ( $K$-band). Telluric corrections have been applied to the spectra as discussed in Section 2.2. H I and He I emission features are commonly observed in the accreting stars, while no star exhibits detectable $\mathrm{H}_{2} 2.12 \mu \mathrm{m}$ emission. L40 is the only star with apparent $\mathrm{Br} 10-13$ emission in $H$-band. Atomic lines and molecular features found useful in classification are identified. Relevant standards from the IRTF spectral atlas (Rayner et al. 2008, in preparation) are shown for comparison (the resolution for the IC 348 observations is $R \sim 1500$ and that of the spectral atlas, $R \sim 2000$ ).

\section{REFERENCES}

Alexander, R. D., Clarke, C. J., \& Pringle, J. E. 2006, MNRAS, 369, 216 Baraffe, I., Chabrier, G., Allard, F., \& Hauschildt, P. H. 1998, A\&A, 337, 403 Basri, G., \& Marcy, G. W. 1995, AJ, 109, 762

Batalha, C. C., \& Basri, G. 1993, ApJ, 412, 363

Beristain, G., Edwards, S., \& Kwan, J. 2001, ApJ, 551, 1037

Cabrit, S., Edwards, S., Strom, S. E., \& Strom, K. M. 1990, ApJ, 354, 687

Calvet, N., \& Gullbring, E. 1998, ApJ, 509, 802

Carpenter, J. M. 2002, AJ, 124, 1593

Carpenter, J. M., Mamajek, E. E., Hillenbrand, L. A., \& Meyer, M. R. 2006, ApJ, 651, L49

Cushing, M. C., Vacca, W. D., \& Rayner, J. T. 2004, PASP, 116, 362

D’Alessio, P. D., Calvet, N., Hartmann, L., Franco-Hernandez, R., \& Servin, H. 2006, ApJ, 638, 314

Dahm, S. E., \& Hillenbrand, L. A. 2007, AJ, 133, 2072

Edwards, S., Fischer, W., Hillenbrand, L. A., \& Kwan, J. 2006, ApJ, 646, 319

Edwards, S., Fischer, W., Kwan, J., Hillenbrand, L. A., \& Dupree, A. K. 2003, ApJ L41

Edwards, S., Hartigan, P., Ghandour, L., \& Andrulis, C. 1994, AJ, 108, 1056

Eisloffel, J., Froebrich, D., Stanke, T., \& McCaughrean, M. J. 2003, ApJ, 595,259

Fischer, W., Edwards, S., Kwan, J., \& Hillenbrand, L. A. 2007, Keck Science Meeting

Gullbring, E., Hartmann, L., Briceno, C., \& Calvet, N. 1998, ApJ, 492, 323

Hamann, F., \& Persson, S. E. 1992, ApJS, 82, 247

Hartigan, P., \& Kenyon, S. J. 2003, ApJ, 683, 334

Hartmann, L., Calvet, N., Gullbring, E., \& D’Alessio, P. 1998, ApJ, 495, 385

Hauschildt, P. H., Allard, F., \& Baron, E. 1999, ApJ, 512, 377

Herbig, G. H. 1954, AJ, PASP, 66, 19
Herbig, G. H. 1998, ApJ, 497, 736

Herczeg, G., \& Hillenbrand, L. A. 2008, ApJ, in press

Hillenbrand, L. A. 1997, AJ, 113, 1733

Kenyon, S. J., Dobrzycka, D., \& Hartmann, L. 1994, AJ, 108, 1872

Kenyon, S. J., \& Hartmann, L. 1995, ApJS, 101, 117

Kurosawa, R., Harries, T. J., \& Syminton, N. H. 2006, MNRAS, 370, 580

Lada, E. A., \& Lada, C. J. 1995, AJ, 109, 1682

Lada, C. J., et al. 2006, AJ, 131, 1574

Luhman, K. L. 1999, ApJ, 525, 466

Luhman, K. L., Rieke, G. H., Lada, C. J., \& Lada, E. A. 1998, ApJ, 508, 347

Luhman, K. L., Stauffer, J. R., Muench, A. A., Rieke, G. H., Lada, E. A., Bouvier, J., \& Lada, C. J. 2003, ApJ, 593, 1093

Marcy, G., Butler, R. P., Fischer, D., Vogt, S., Wright, J. T., Tinney, C. G., \& Jones, H. R. A. 2005, in Progress of Theoretical Physics Supplement, 158,24

Martín, E. L. 1998, AJ, 115, 351

Martin, P. G., \& Whittet, D. C. B. 1990, ApJ, 357, 113

Meyer, M. R., Edwards, S., Hinkle, K. H., \& Strom, S. E. 1998, ApJ, 508, 397

Muench, A. A., Lada, C. J., Luhman, K. L., Muzerolle, J., \& Young, E. 2007, AJ, 134,411

Muench, A. A., et al. 2003, AJ, 125, 2029

Muzerolle, J., Hartmann, L., \& Calvet, N. 1998a, AJ, 116, 455

Muzerolle, J., Hartmann, L., \& Calvet, N. 1998b, ApJ, 492, 743

Muzerolle, J., Hartmann, L., \& Calvet, N. 1998c, AJ, 116, 2965

Muzerolle, J., Hartmann, L., \& Calvet, N. 2001, ApJ, 550, 944

Najita, J. R., Strom, S. E., \& Muzerolle, J. 2007, MNRAS, 378, 369

Nordhagen, S., Herbst, W., Rhode, K. L., \& Williams, E. C. 2006, AJ, 132,1555

O'Connell, R. W. 1973, AJ, 78, 1074

Preibisch, T., \& Zinnecker, H. 2001, AJ, 122, 866

Preibisch, T., \& Zinnecker, H. 2002, AJ, 123, 1613

Preibisch, T., Zinnecker, H., \& Herbig, G. H. 1996, A\&A, 310, 456

Rayner, J. T., Toomey, D. W., Onaka, P. M., Denault, A. J., Stahlberger, W. E., Vacca, W. D., Cushing, M. C., \& Wang, S. 2003, PASP, 115, 362

Reipurth, B., Heathcote, S., Morse, J., Hartigan, P., \& Bally, J. 2002, AJ, 123,362

Siess, L., Dufour, E., \& Forestini, M. 2000, A\&A, 358, 593

Silverstone, M. D., et al. 2006, ApJ, 639, 1138

Snow, T. P., Hanson, M., Seab, G. C., \& Saken, J. M. 1994, ApJ, 420, 632

Soderblom, D. R., Jones, B. F., Balachandran, S., Stauffer, J. R., Duncan, D. K., Fedele, S. B., \& Hudon, J. D. 1993, AJ, 106, 1059

Song, I., Bessell, M. S., \& Zuckerman, B. 2002, ApJ, 581, L43

Sung, H., Bessell, M. S., \& Chun, M. Y. 2004, AJ, 128, 1684

Tokunaga, A. T. 2000, in Allen's Astrophysical Quantities, ed. A. N. Cox (4th edn; New York: Springer), 143

Uchida, K. I., et al. 2004, ApJS, 154, 539

Vacca, W. D., Cushing, M. C., \& Rayner, J. T. 2003, PASP, 115, 389

Valenti, J. A., Basri, G., \& Johns, C. M. 1993, AJ, 106, 2024

Vogt, S. S., et al. 1994, SPIE, 2198, 362

Wallace, L., Meyer, M. R., Hinkle, K., \& Edwards, S. 2000, ApJ, 535, 325

White, R. J., \& Basri, G. 2003, ApJ, 582, 1109

White, R. J., \& Hillenbrand, L. A. 2004, ApJ, 616, 998 